\title{
THE SIX OPERATIONS FOR SHEAVES ON ARTIN STACKS I: FINITE COEFFICIENTS
}

\author{
YVES LASZLO AND MARTIN OLSSON
}

\begin{abstract}
In this paper we develop a theory of Grothendieck's six operations of lisse-étale constructible sheaves on Artin stacks locally of finite type over an affine regular noetherian scheme of dimension $\leq 1$. We also generalize the classical base change theorems and Kunneth formula to stacks, and prove new results about cohomological descent for unbounded complexes.
\end{abstract}

\section{INTRODUCTION}

We denote by $\Lambda$ a Gorenstein local ring of dimension 0 and characteristic $l$. Let $\mathrm{S}$ be an affine regular, noetherian scheme of dimension $\leq 1$ and assume $l$ is invertible on $\mathrm{S}$. We assume that all S-schemes of finite type $\mathrm{X}$ satisfy $\operatorname{cd}_{l}(\mathrm{X})<\infty$ (see 1.0.1 for more discussion of this). For an algebraic stack $\mathscr{X}$ locally of finite type over $\mathrm{S}$ and $* \in\{+,-, b, \emptyset,[a, b]\}$ we write $\mathrm{D}_{c}^{*}(\mathscr{X})$ for the full subcategory of the derived category $\mathrm{D}^{*}(\mathscr{X})$ of complexes of $\Lambda$-modules on the lisse-étale site of $\mathscr{X}$ with constructible cohomology sheaves.

In this paper we develop a theory of Grothendieck's six operations of lisse-étale constructible sheaves on Artin stacks locally of finite type over $\mathrm{S}^{1}$. In forthcoming papers, we will also develop a theory of adic sheaves and perverse sheaves for Artin stacks. In addition to being of basic foundational interest, we hope that the development of these six operations for stacks will have a number of applications. Already the work done in this paper (and the forthcoming ones) provides the necessary tools needed in several papers on the geometric Langland's program (e.g. [17], 15], 11]). We hope that it will also shed further light on the Lefschetz trace formula for stacks proven by Behrend ([ $[\underline{6})$, and also to versions of such a formula for stacks not necessarily of finite type. We should also remark that recent work of Toen should provide another approach to defining the six operations for stacks, and in fact should generalize to a theory for $n$-stacks.

Let us describe more precisely the contents of this papers. For a morphism $f: \mathscr{X} \rightarrow \mathscr{Y}$ of such S-stacks we define functors

$$
\mathrm{R} f_{*}: \mathrm{D}_{c}^{+}(\mathscr{X}) \rightarrow \mathrm{D}_{c}^{+}(\mathscr{Y}), \quad \mathrm{R} f_{!}: \mathrm{D}_{c}^{-}(\mathscr{X}) \rightarrow \mathrm{D}_{c}^{-}(\mathscr{Y})
$$

\footnotetext{
${ }^{1}$ In fact our method could apply to other situations like analytic stacks or non separated analytic varieties.
} 


$$
\begin{gathered}
\mathrm{L} f^{*}: \mathrm{D}_{c}(\mathscr{Y}) \rightarrow \mathrm{D}_{c}(\mathscr{X}), \quad \mathrm{R} f^{!}: \mathrm{D}_{c}(\mathscr{Y}) \rightarrow \mathrm{D}_{c}(\mathscr{X}), \\
\mathscr{R} h o m: \mathrm{D}_{c}^{-}(\mathscr{X})^{\mathrm{op}} \times \mathrm{D}_{c}^{+}(\mathscr{X}) \rightarrow \mathrm{D}_{c}^{+}(\mathscr{X}),
\end{gathered}
$$

and

$$
(-) \stackrel{\mathbf{L}}{\otimes}(-): \mathrm{D}_{c}^{-}(\mathscr{X}) \times \mathrm{D}_{c}^{-}(\mathscr{X}) \rightarrow \mathrm{D}_{c}^{-}(\mathscr{X})
$$

satisfying all the usual adjointness properties that one has in the theory for schemes ${ }^{2}$.

The main tool is to define $\mathrm{R} f$ !,$f^{!}$, even for unbounded constructible complexes, by duality. One of the key points is that, as observed by Laumon, the dualizing complex is a local object of the derived category and hence has to exist for stacks by glueing (see 2.3.3). Notice that this formalism applies to non-separated schemes, giving a theory of cohomology with compact supports in this case. Previously, Laumon and Moret-Bailly constructed the truncations of dualizing complexes for Bernstein-Lunts stacks (see [16]). Our constructions reduces to theirs in this case. Another approach using a dual version of cohomological descent has been suggested by Gabber but seems to be technically much more complicated.

Remark 1.0.1. The cohomological dimension hypothesis on schemes of finite type over $\mathrm{S}$ is achieved for instance if $\mathrm{S}$ is the spectrum of a finite field or of a separably closed field. In dimension 1, it will be achieved for instance for the spectrum of a complete discrete valuation field with residue field either finite or separably closed, or if $\mathrm{S}$ is a smooth curve over $\mathbf{C}, \mathbf{F}_{q}$ (cf. [4, exp. $\mathrm{X}$ and [21]). In these situations, $\mathrm{cd}_{l}(\mathrm{X})$ is bounded by a function of the dimension $\operatorname{dim}(\mathrm{X})$. Notice that, as pointed out by Illusie, recent results of Gabber enables one to dramatically weaken the hypothesis on S. Unfortunately no written version of these results seems to be available at this time.

1.1. Conventions. Recall that for any ring $\mathscr{O}$ of a topos, the category of complexes of $\mathscr{O}_{-}$ modules has enough K-injective (or homotopically injective). Recall that a complex I is $\mathrm{K}$ injective if for any acyclic complex of $\mathscr{O}$-modules A, the complex $\mathscr{H}$ om (A, I) is acyclic (see [22). For instance, any injective resolution in the sense of Cartan-Eileberg of a bounded below complex is K-injective. This result is due, at least for sheaves on a topological space to [22] and enables him to extend the formalism of direct images and $\mathscr{R} h o m$ to unbounded complexes. But this result is true for any Grothendieck category $([20])$. Notice that the category of $\mathscr{O}$-modules has enough K-flat objects, enabling one to define $\stackrel{\mathbf{L}}{\otimes}$ for unbounded objects $([22])$.

All the stacks we will consider will be locally of finite type over S. As in [16], lemme 12.1.2, the lisse-étale topos $\mathscr{X}_{\text {lis-ét }}$ can be defined using the site Lisse-Et $(\mathscr{X})$ whose objects

${ }^{2}$ We will often write $f^{*}, f^{!}, f_{*}, f_{!}$for $\mathrm{L} f^{*}, \mathrm{R} f^{!}, \mathrm{R} f_{*}, \mathrm{R} f_{!}$. 
THE SIX OPERATIONS FOR SHEAVES ON ARTIN STACKS I: FINITE COEFFICIENTS

are S-morphisms $u: \mathrm{U} \rightarrow \mathscr{X}$ where $\mathrm{U}$ is an algebraic space which is separated and of finite type over $\mathrm{S}$. The topology is generated by the pretopology such that the covering families are finite families $\left(\mathrm{U}_{i}, u_{i}\right) \rightarrow(\mathrm{U}, u)$ such that $\bigsqcup \mathrm{U}_{i} \rightarrow \mathrm{U}$ is surjective and étale (use the comparison theorem [2], III.4.1 remembering $\mathscr{X}$ is locally of finite type over S). Notice that products over $\mathscr{X}$ are representable in Lisse-Et $(\mathscr{X})$, simply because the diagonal morphism $\mathscr{X} \rightarrow \mathscr{X} \times{ }_{\mathrm{S}} \mathscr{X}$ is representable by definition ([16]).

If $\mathrm{C}$ is a complex of sheaves and $d$ a locally constant valued function $\mathrm{C}(d)$ is the Tate twist and $\mathrm{C}[d]$ the shifted complex. We denote $\mathrm{C}(d)[2 d]$ by $\mathrm{C}\langle d\rangle$. Let $\Omega=\Lambda\langle\operatorname{dim}(\mathrm{S})\rangle$ be the dualizing complex of S ([9], "Dualité").

\section{Homological algebra}

2.1. Existence of $\mathrm{K}$-injectives. Let $(\mathscr{S}, \mathscr{O})$ denote a ringed site, and let $\mathscr{C}$ denote a full subcategory of the category of $\mathscr{O}$-modules on $\mathscr{S}$. Let $\mathrm{M}$ be a complex of $\mathscr{O}$-modules on $\mathscr{S}$. By $(22,3.7)$ there exists a morphism of complexes $f: \mathrm{M} \rightarrow \mathrm{I}$ with the following properties:

(i) $\mathrm{I}=\lim _{n} \mathrm{I}_{n}$ where each $\mathrm{I}_{n}$ is a bounded below complex of flasque $\mathscr{O}$-modules.

(ii) The morphism $f$ is induced by a compatible collection of quasi-isomorphisms $f_{n}$ : $\tau_{\geq-n} \mathrm{M} \rightarrow \mathrm{I}_{n}$

(iii) For every $n$ the map $\mathrm{I}_{n} \rightarrow \mathrm{I}_{n-1}$ is surjective with kernel $\mathrm{K}_{n}$ a bounded below complex of flasque $\mathscr{O}$-modules.

(iv) For any pair of integers $n$ and $i$ the sequence

$$
0 \rightarrow \mathrm{K}_{n}^{i} \rightarrow \mathrm{I}_{n}^{i} \rightarrow \mathrm{I}_{n-1}^{i} \rightarrow 0
$$

is split.

Remark 2.1.1. In fact $\left([22,3.7)\right.$ shows that we can choose $\mathrm{I}_{n}$ and $\mathrm{K}_{n}$ to be complexes of injective $\mathscr{O}$-modules (in which case (iv) follows from (iii)). However, for technical reasons it is sometimes useful to know that one can work just with flasque sheaves.

We make the following finiteness assumption, which is the analog of [22], $3.12(1)$.

Assumption 2.1.2. For any object $U \in \mathscr{S}$ there exists a covering $\left\{\mathrm{U}_{i} \rightarrow U\right\}_{i \in \mathrm{I}}$ and an integer $n_{0}$ such that for any sheaf of $\mathscr{O}$-modules $\mathrm{F} \in \mathscr{C}$ we have $\mathrm{H}^{n}\left(\mathrm{U}_{i}, \mathrm{~F}\right)=0$ for all $n \geq n_{0}$.

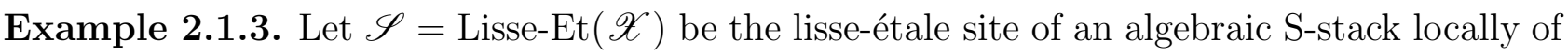
finite type $\mathscr{X}$ and $\mathscr{O}$ a constant local Artinian ring of characteristic $\Lambda$ invertible on $\mathrm{S}$. Then the class $\mathscr{C}$ of all $\mathscr{O}$-sheaves, cartesian or not, satisfies the assumption. Indeed, if $\mathrm{U} \in \mathscr{S}$ is of 
finite type over $\mathrm{S}$ and $\mathrm{F} \in \mathscr{S}$, one has $\mathrm{H}^{n}(\mathrm{U}, \mathrm{F})=\mathrm{H}^{n}\left(\mathrm{U}_{\text {ét }}, \mathrm{F}_{\mathrm{U}}\right)^{3}$ which is zero for $n$ bigger than a constant depending only on $\mathrm{U}$ (and not on F). Therefore, one can take the trivial covering in this case. We could also take $\mathscr{O}=\mathscr{O}_{\mathscr{X}}$ and $\mathscr{C}$ to be the class of quasi-coherent sheaves.

With hypothesis 2.1.2, one has the following criterion for $f$ being a quasi-isomorphism (cf. [22], 3.13).

Proposition 2.1.4. Assume that $\mathscr{H}^{j}(\mathrm{M}) \in \mathscr{C}$ for all $j$. Then the map $f$ is a quasi-isomorphism. In particular, if each $\mathrm{I}_{n}$ is a complex of injective $\mathscr{O}$-modules then by [22, 2.5, $f: \mathrm{M} \rightarrow \mathrm{I}$ is a $\mathrm{K}$-injective resolution of $\mathrm{M}$.

Proof: For a fixed integer $j$, the map $\mathscr{H}^{j}(\mathrm{M}) \rightarrow \mathscr{H}^{j}\left(\mathrm{I}_{n}\right)$ is an isomorphism for $n$ sufficiently big. Since this isomorphism factors as

$$
\mathscr{H}^{j}(\mathrm{M}) \rightarrow \mathscr{H}^{j}(\mathrm{I}) \rightarrow \mathscr{H}^{j}\left(\mathrm{I}_{n}\right)
$$

it follows that the map $\mathscr{H}^{j}(\mathrm{M}) \rightarrow \mathscr{H}^{j}(\mathrm{I})$ is injective.

To see that $\mathscr{H}^{j}(\mathrm{M}) \rightarrow \mathscr{H}^{j}(\mathrm{I})$ is surjective, let $\mathrm{U} \in \mathscr{S}$ be an object and $\gamma \in \Gamma\left(\mathrm{U}, \mathrm{I}^{j}\right)$ an element with $d \gamma=0$ defining a class in $\mathscr{H}^{j}(\mathrm{I})(\mathrm{U})$. Since $\mathrm{I}=\lim _{\longleftarrow} \mathrm{I}_{n}$ the class $\gamma$ is given by a compatible collection of sections $\gamma_{n} \in \Gamma\left(\mathrm{U}, \mathrm{I}_{n}^{j}\right)$ with $d \gamma_{n}=0$.

Let $\left(\mathscr{U}=\left\{\mathrm{U}_{i} \rightarrow \mathrm{U}\right\}, n_{0}\right)$ be the data provided by 2.1.2. Let $\mathrm{N}$ be an integer greater than $n_{0}-j$. For $m>\mathrm{N}$ and $\mathrm{U}_{i} \in \mathscr{U}$ the sequence

$$
\Gamma\left(\mathrm{U}_{i}, \mathrm{~K}_{m}^{j-1}\right) \rightarrow \Gamma\left(\mathrm{U}_{i}, \mathrm{~K}_{m}^{j}\right) \rightarrow \Gamma\left(\mathrm{U}_{i}, \mathrm{~K}_{m}^{j+1}\right) \rightarrow \Gamma\left(\mathrm{U}_{i}, \mathrm{~K}_{m}^{j+2}\right)
$$

is exact. Indeed $\mathrm{K}_{m}$ is a bounded below complex with $\mathscr{H}^{j}\left(\mathrm{~K}_{m}\right) \in \mathscr{C}$ for every $j$ and $\mathscr{H}^{j}\left(\mathrm{~K}_{m}\right)=$ 0 for $j \geq-m+2$. It follows that $\mathrm{H}^{j}\left(\mathrm{U}_{i}, \mathrm{~K}_{m}\right)=0$ for $j \geq n_{0}-m+2$.

Since the maps $\Gamma\left(\mathrm{U}_{i}, \mathrm{I}_{m}^{r}\right) \rightarrow \Gamma\left(\mathrm{U}_{i}, \mathrm{I}_{m-1}^{r}\right)$ are also surjective for all $m$ and $r$, it follows from $(22], 0.11)$ applied to the system

$$
\Gamma\left(\mathrm{U}_{i}, \mathrm{I}_{m}^{j-1}\right) \rightarrow \Gamma\left(\mathrm{U}_{i}, \mathrm{I}_{m}^{i}\right) \rightarrow \Gamma\left(\mathrm{U}_{i}, \mathrm{I}_{m}^{j+1}\right) \rightarrow \Gamma\left(\mathrm{U}_{i}, \mathrm{I}_{m}^{j+2}\right)
$$

that the map

$$
\mathrm{H}^{j}\left(\Gamma\left(\mathrm{U}_{i}, \mathrm{I}\right)\right) \rightarrow \mathrm{H}^{j}\left(\Gamma\left(\mathrm{U}_{i}, \mathrm{I}_{m}\right)\right)
$$

is an isomorphism.

Then since the map $\mathscr{H}^{j}(\mathrm{M}) \rightarrow \mathscr{H}^{j}\left(\mathrm{I}_{m}\right)$ is an isomorphism it follows that for every $i$ the restriction of $\gamma$ to $\mathrm{U}_{i}$ is in the image of $\mathscr{H}^{j}(\mathrm{M})\left(\mathrm{U}_{i}\right)$.

\footnotetext{
${ }^{3} \mathrm{Cf}$. 3.3.1 below
} 
Next consider a fibred topos $\mathscr{T} \rightarrow \mathrm{D}$ with corresponding total topos $\mathscr{T} \bullet([3]$, VI.7). We call $\mathscr{T}$ a D -simplicial topos. Concretely, this means that for each $i \in \mathrm{D}$ the fiber $\mathscr{T}_{i}$ is a topos and that any $\delta \in \operatorname{Hom}_{\mathrm{D}}(i, j)$ comes together with a morphism of topos $\delta: \mathscr{T}_{i} \rightarrow \mathscr{T}_{j}$ such that $\delta^{-1}$ is the inverse image functor of the fibred structure. The objects of the total topos are simply collections $\left(\mathrm{F}_{i} \in \mathrm{E}_{i}\right)_{i \in \mathrm{D}}$ together with functorial transition morphisms $\delta^{-1} \mathrm{~F}_{j} \rightarrow \mathrm{F}_{i}$ for any $\delta \in \operatorname{Hom}_{\mathrm{D}}(i, j)$. We assume furthermore that $\mathscr{T}_{\bullet}$ is ringed by a $\mathscr{O}_{\bullet}$ and that for any $\delta \in \operatorname{Hom}_{\mathrm{D}}(i, j)$, the morphism $\delta:\left(\mathscr{T}_{i}, \mathscr{O}_{i}\right) \rightarrow\left(\mathscr{T}_{j}, \mathscr{O}_{j}\right)$ is flat.

Example 2.1.5. Let $\Delta^{+}$be the category whose objects are the ordered sets $[n]=\{0, \ldots, n\}$ $(n \in \mathbb{N})$ and whose morphisms are injective order-preserving maps. Let $\mathrm{D}$ be the opposite category of $\Delta^{+}$. In this case $\mathscr{T}$. is called a strict simplicial topos. For instance, if $\mathrm{U} \rightarrow \mathscr{X}$ is a presentation, the simplicial algebraic space $\mathrm{U}_{\bullet}=\operatorname{cosq}_{0}(\mathrm{U} / \mathscr{X})$ defines a strict simplicial

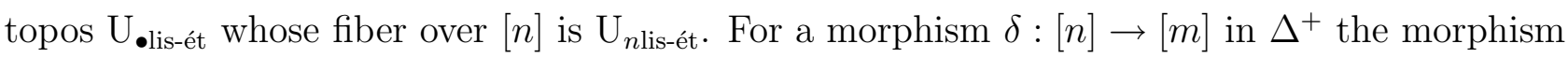
$\delta: \mathscr{T}_{m} \rightarrow \mathscr{T}_{n}$ is induced by the (smooth) projection $\mathrm{U}_{m} \rightarrow \mathrm{U}_{n}$ defined by $\delta \in \operatorname{Hom}_{\Delta^{+o p p}}([m],[n])$.

Example 2.1.6. Let $\mathbf{N}$ be the natural numbers viewed as a category in which $\operatorname{Hom}(n, m)$ is empty unless $m \geq n$ in which case it consists of a unique element. For a topos $\mathrm{T}$ we can then define an $\mathbf{N}$-simplicial topos $\mathrm{T}^{\mathbf{N}}$. The fiber over $n$ of $\mathrm{T}^{\mathbf{N}}$ is $\mathrm{T}$ and the transition morphisms by the identity of $\mathrm{T}$. The topos $\mathrm{T}^{\mathbf{N}}$ is the category of projective systems in $\mathrm{T}$. If $\mathscr{O} \bullet$ is a constant projective system of rings then the flatness assumption is also satisfied, or more generally if $\delta^{-1} \mathscr{O}_{n} \rightarrow \mathscr{O}_{m}$ is an isomorphism for any morphism $\delta: m \rightarrow n$ in $\mathbb{N}$ then the flatness assumption holds.

Let $\mathscr{C}_{\bullet}$ be a full subcategory of the category of $\mathscr{O}_{\bullet}-$ modules on a ringed D-simplicial topos $\left(\mathscr{T}_{\bullet}, \mathscr{O}_{\bullet}\right)$. For $i \in \mathrm{D}$, let $e_{i}: \mathscr{T}_{n} \rightarrow \mathscr{T}_{\bullet}$ the morphism of topos defined by $e_{i}^{-1} \mathrm{~F}_{\bullet}=\mathrm{F}_{n}$ (cf. [3], Vbis, 1.2.11). Recall that the family $e_{i}^{-1}, i \in \mathrm{D}$ is conservative. Let $\mathscr{C}_{i}$ denote the essential image of $\mathscr{C}_{\bullet}$ under $e_{i}^{-1}$ (which coincides with $e_{i}^{*}$ on $\operatorname{Mod}\left(\mathscr{T}_{\bullet}, \mathscr{O}_{\bullet}\right)$ because $\left.e_{i}^{-1} \mathscr{O}_{\bullet}=\mathscr{O}_{i}\right)$.

Assumption 2.1.7. For every $i \in \mathrm{D}$ the ringed topos $\left(\mathscr{T}_{i}, \mathscr{O}_{i}\right)$ is isomorphic to the topos of a ringed site satisfying 2.1 .2 with respect to $\mathscr{C}_{i}$.

Example 2.1.8. Let $\mathscr{T}$ be the topos $\left(\mathscr{X}_{\text {lis-ét }}\right)^{\mathbf{N}}$ of a S-stack locally of finite type. Then, the full subcategory $\mathscr{C}_{\bullet}$ of $\operatorname{Mod}\left(\mathscr{T}_{\bullet}, \mathscr{O}_{\bullet}\right)$ whose objects are families $\mathrm{F}_{i}$ of cartesian modules satisfies the hypothesis.

Let $\mathrm{M}$ be a complex of $\mathscr{O}_{\bullet}-$ modules on $\mathscr{T}$. Again by $([22,3.7)$ there exists a morphism of complexes $f: \mathrm{M} \rightarrow \mathrm{I}$ with the following properties: 
(S i) $\mathrm{I}=\varliminf_{\varliminf} \mathrm{I}_{n}$ where each $\mathrm{I}_{n}$ is a bounded below complex of injective modules.

(S ii) The morphism $f$ is induced by a compatible collection of quasi-isomorphisms $f_{n}$ : $\tau_{\geq-n} \mathrm{M} \rightarrow \mathrm{I}_{n}$.

(S iii) For every $n$ the map $\mathrm{I}_{n} \rightarrow \mathrm{I}_{n-1}$ is surjective with kernel $\mathrm{K}_{n}$ a bounded below complex of injective $\mathscr{O}$-modules.

(S iv) For any pair of integers $n$ and $i$ the sequence

$$
0 \rightarrow \mathrm{K}_{n}^{i} \rightarrow \mathrm{I}_{n}^{i} \rightarrow \mathrm{I}_{n-1}^{i} \rightarrow 0
$$

is split.

Proposition 2.1.9. Assume that $\mathscr{H}^{j}(\mathrm{M}) \in \mathscr{C} \bullet$ for all $j$. Then the morphism $f$ is a quasiisomorphism and $f: \mathrm{M} \rightarrow \mathrm{I}$ is a $\mathrm{K}$-injective resolution of $\mathrm{M}$.

Proof: By [22], 2.5, it suffices to show that $f$ is a quasi-isomorphism. For this in turn it suffices to show that for every $i \in \mathrm{D}$ the restriction $e_{i}^{*} f: e_{i}^{*} \mathrm{M} \rightarrow e_{i}^{*} \mathrm{I}$ is a quasiisomorphism of complexes of $\mathscr{O}_{i}$-modules since the family $e_{i}^{*}=e_{i}^{-1}$ is conservative. But $e_{i}^{*}: \operatorname{Mod}\left(\mathscr{T}_{\bullet}, \mathscr{O}_{\bullet}\right) \rightarrow \operatorname{Mod}\left(\mathscr{T}_{i}, \mathscr{O}_{i}\right)$ has a left adjoint $e_{i !}$ defined by

$$
\left[e_{i !}(\mathrm{F})\right]_{j}=\oplus_{\delta \in \operatorname{Hom}_{\mathrm{D}}(j, i)} \delta^{*} \mathrm{~F}
$$

with the obvious transition morphisms. It is exact by the flatness of the morphisms $\delta$. It follows that $e_{i}^{*}$ takes injectives to injectives and commutes with direct limits. We can therefore apply 2.1.4 to $e_{i}^{*} \mathrm{M} \rightarrow e_{i}^{*} \mathrm{I}$ to deduce that this map is a quasi-isomorphism.

In what follows we call a $\mathrm{K}$-injective resolution $f: \mathrm{M} \rightarrow \mathrm{I}$ obtained from data (i)-(iv) as above a Spaltenstein resolution.

The main technical lemma is the following.

Lemma 2.1.10. Let $\epsilon:\left(\mathscr{T}_{\bullet}, \mathscr{O}_{\bullet}\right) \rightarrow(\mathrm{S}, \Psi)$ be a morphism of ringed topos, and let $\mathrm{C}$ be a complex of $\mathscr{O}_{\bullet}$-modules. Assume that

(1) $\mathscr{H}^{n}(\mathrm{C}) \in \mathscr{C}_{\bullet}$ for all $n$.

(2) There exists $i_{0}$ such that $\mathrm{R}^{i} \epsilon_{*} \mathscr{H}^{n}(\mathrm{C})=0$ for any $n$ and any $i>i_{0}$.

Then, if $j \geq-n+i_{0}$, we have $\mathrm{R}^{j} \epsilon_{*} \mathrm{C}=\mathrm{R}^{j} \epsilon_{*} \tau_{\geq-n} \mathrm{C}$.

Proof: By 2.1.9 and assumption (1), there exists a Spaltenstein resolution $f: \mathrm{C} \rightarrow \mathrm{I}$ of $\mathrm{C}$. Let $\mathrm{J}_{n}:=\epsilon_{*} \mathrm{I}_{n}$ and $\mathrm{D}_{n}:=\epsilon_{*} \mathrm{~K}_{n}$. Since the sequences 2.1.8.1 are split, the sequences

$$
0 \rightarrow \mathrm{D}_{n} \rightarrow \mathrm{J}_{n} \rightarrow \mathrm{J}_{n-1} \rightarrow 0
$$


are exact.

The exact sequence 2.1.8.1 and property (S ii) defines a distinguished triangle

$$
\mathrm{K}_{n} \rightarrow \tau_{\geq-n} \mathrm{C} \rightarrow \tau_{\geq-n+1} \mathrm{C}
$$

showing that $\mathrm{K}_{n}$ is quasi-isomorphic to $\mathscr{H}^{-n}(\mathrm{C})[n]$. Because $\mathrm{K}_{n}$ is a bounded below complex of injectives, one gets

$$
\mathrm{R} \epsilon_{*} \mathscr{H}^{-n}(\mathrm{C})[n]=\epsilon_{*} \mathrm{~K}_{n}
$$

and accordingly

$$
\mathrm{R}^{j+n} \epsilon_{*} \mathscr{H}^{-n}(\mathrm{C})=\mathscr{H}^{j}\left(\epsilon_{*} \mathrm{~K}_{n}\right)=\mathscr{H}^{j}\left(\mathrm{D}_{n}\right)
$$

By assumption (2), we have therefore

$$
\mathscr{H}^{j}\left(\mathrm{D}_{n}\right)=0 \text { for } j>-n+i_{0}
$$

By $(22,0.11)$ this implies that

$$
\mathscr{H}^{j}\left(\lim _{\longleftarrow} \mathrm{J}_{n}\right) \rightarrow \mathscr{H}^{j}\left(\mathrm{~J}_{n}\right)
$$

is an isomorphism for $j \geq-n+i_{0}$. But, by adjunction, $\epsilon_{*}$ commutes with projective limit. In particular, one has

$$
\lim _{n} \mathrm{~J}_{n}=\epsilon_{*} \mathrm{I}
$$

and by (S i) and (S ii)

$$
\mathrm{R} \epsilon_{*} \mathrm{C}=\epsilon_{*} \mathrm{I} \text { and } \mathrm{R} \epsilon_{*} \tau_{\geq-n} \mathrm{C}=\epsilon_{*} \mathrm{~J}_{n}
$$

Thus for any $n$ such that $j \geq-n+i_{0}$ one has

$$
\mathrm{R}^{j} \epsilon_{*} \mathrm{C}=\mathscr{H}^{j}\left(\epsilon_{*} \mathrm{I}\right)=\mathscr{H}^{j}\left(\mathrm{~J}_{n}\right)=\mathrm{R}^{j} \epsilon_{*} \tau_{\geq-n} \mathrm{C} .
$$

2.2. The descent theorem. Let $\left(\mathscr{T}_{\bullet}, \mathscr{O}_{\bullet}\right)$ be a simplicial or strictly simplicial ${ }^{4}$ ringed topos $\left(\mathrm{D}=\Delta^{\mathrm{opp}}\right.$ or $\left.\mathrm{D}=\Delta^{\text {opp }}\right)$, let $(\mathrm{S}, \Psi)$ be another ringed topos, and let $\epsilon:\left(\mathscr{T}_{\bullet}, \mathscr{O}_{\bullet}\right) \rightarrow(\mathrm{S}, \Psi)$ be an augmentation. Assume that $\epsilon$ is a flat morphism (i.e. for every $i \in \mathrm{D}$, the morphism of ringed topos $\left(\mathscr{T}_{i}, \mathscr{O}_{i}\right) \rightarrow(\mathrm{S}, \Psi)$ is a flat morphism $)$.

Let $\mathscr{C}$ be a full subcategory of the category of $\Psi$-modules, and assume that $\mathscr{C}$ is closed under kernels, cokernels and extensions (one says that $\mathscr{C}$ is a Serre subcategory). Let $\mathrm{D}(\mathrm{S})$ denote the derived category of $\Psi$-modules, and let $\mathrm{D}_{\mathscr{C}}(\mathrm{S}) \subset \mathrm{D}(\mathrm{S})$ be the full subcategory

\footnotetext{
${ }^{4}$ One could replace simplicial by multisimplicial
} 
consisting of complexes whose cohomology sheaves are in $\mathscr{C}$. Let $\mathscr{C} \bullet$ denote the essential image of $\mathscr{C}$ under the functor $\epsilon^{*}: \operatorname{Mod}(\Psi) \rightarrow \operatorname{Mod}\left(\mathscr{O}_{\bullet}\right)$.

We assume the following condition holds:

Assumption 2.2.1. Assumption 2.1.7 holds (with respect to $\mathscr{C}_{\bullet}$ ), and $\epsilon^{*}: \mathscr{C} \rightarrow \mathscr{C}_{\bullet}$ is an equivalence of categories with quasi-inverse $R \epsilon_{*}$.

Lemma 2.2.2. The full subcategory $\mathscr{C}_{\bullet} \subset \operatorname{Mod}\left(\mathscr{O}_{\bullet}\right)$ is closed under extensions, kernels and cokernels.

Proof: Consider an extension of sheaves of $\mathscr{O}_{\bullet}-$ modules

$$
0 \longrightarrow \epsilon^{*} \mathrm{~F}_{1} \longrightarrow \mathrm{E} \longrightarrow \epsilon^{*} \mathrm{~F}_{2} \longrightarrow 0
$$

where $\mathrm{F}_{1}, \mathrm{~F}_{2} \in \mathscr{C}$. Since $\mathrm{R}^{1} \epsilon_{*} \epsilon^{*} \mathrm{~F}_{1}=0$ and the maps $\mathrm{F}_{i} \rightarrow \mathrm{R}^{0} \epsilon_{*} \epsilon^{*} \mathrm{~F}_{i}$ are isomorphisms, we obtain by applying $\epsilon^{*} \epsilon_{*}$ a commutative diagram with exact rows

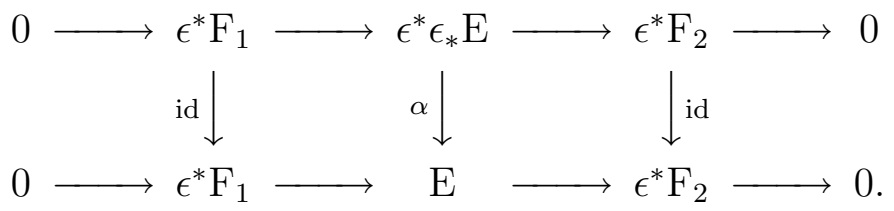

It follows that $\alpha$ is an isomorphism. Furthermore, since $\mathscr{C}$ is closed under extensions we have $\epsilon_{*} \mathrm{E} \in \mathscr{C}$. Let $f \in \operatorname{Hom}\left(\epsilon^{*} \mathrm{~F}_{1}, \epsilon^{*} \mathrm{~F}_{2}\right)$. There exists a unique $\varphi \in \operatorname{Hom}\left(\mathrm{F}_{1}, \mathrm{~F}_{2}\right)$ such that $f=\epsilon^{*} \varphi$. Because $\epsilon^{*}$ is exact, it maps the kernel and cokernel of $\varphi$, which are objects of $\mathscr{C}$, to the kernel and cokernel of $f$ respectively. Therefore, the latter are objects of $\mathscr{C}_{\bullet}$.

Let $\mathrm{D}\left(\mathscr{T}_{\bullet}\right)$ denote the derived category of $\mathscr{O}_{\bullet}-$ modules, and let $\mathrm{D}_{\mathscr{C}_{\bullet}}\left(\mathscr{T}_{\bullet}\right) \subset \mathrm{D}\left(\mathscr{T}_{\bullet}\right)$ denote the full subcategory of complexes whose cohomology sheaves are in $\mathscr{C}_{\bullet}$.

Since $\epsilon$ is a flat morphism, we obtain a morphism of triangulated categories (the fact that these categories are triangulated comes precisely from the fact that both $\mathscr{C}$ and $\mathscr{C} \bullet$ are Serre categories [12]).

$$
\epsilon^{*}: \mathrm{D}_{\mathscr{C}}(\mathrm{S}) \rightarrow \mathrm{D}_{\mathscr{C} \bullet}\left(\mathscr{T}_{\bullet}\right)
$$

Theorem 2.2.3. The functor $\epsilon^{*}$ of 2.2.2.3 is an equivalence of triangulated categories with quasi-inverse given by $\mathrm{R} \epsilon_{*}$.

Proof: Note first that if $\mathrm{M}_{\bullet} \in \mathrm{D}_{\mathscr{C}}\left(\mathscr{T}_{\bullet}\right)$, then by lemma 2.1.10, for any integer $j$ there exists $n_{0}$ such that $\mathrm{R}^{j} \epsilon_{*} \mathrm{M}_{\bullet}=\mathrm{R}^{j} \epsilon_{*} \tau_{\geq n_{0}} \mathrm{M}_{\bullet}$. In particular, we get by induction $\mathrm{R}^{j} \epsilon_{*} \mathrm{M}_{\bullet} \in \mathscr{C}$. Thus $\mathrm{R} \epsilon_{*}$ defines a functor

$$
\mathrm{R}_{*}: \mathrm{D}_{\mathscr{C}}\left(\mathscr{T}_{\bullet}\right) \rightarrow \mathrm{D}_{\mathscr{C}}(\mathrm{S})
$$


To prove 2.2 .3 it suffices to show that for $\mathrm{M}_{\bullet} \in \mathrm{D}_{\mathscr{\bullet}}\left(\mathscr{T}_{\bullet}\right)$ and $\mathrm{F} \in \mathrm{D}_{\mathscr{C}}(\mathrm{S})$ the adjunction maps

$$
\epsilon^{*} \mathrm{R} \epsilon_{*} \mathrm{M}_{\bullet} \rightarrow \mathrm{M}_{\bullet}, \quad \mathrm{F} \rightarrow \mathrm{R} \epsilon_{*} \epsilon^{*} \mathrm{~F}
$$

are isomorphisms. For this note that for any integers $j$ and $n$ there are commutative diagrams

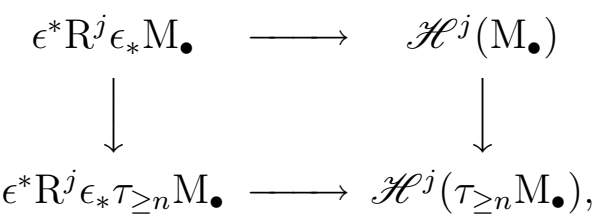

and

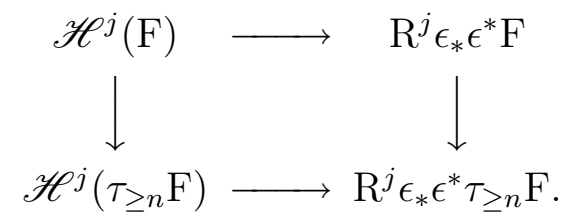

By the observation at the begining of the proof, there exists an integer $n$ so that the vertical arrows in the above diagrams are isomorphisms. This reduces the proof 2.2.3 to the case of a bounded below complex. In this case one reduces by devissage to the case when $\mathrm{M}_{\bullet} \in \mathscr{C}_{\bullet}$ and $\mathrm{F} \in \mathscr{C}$ in which case the result holds by assumption.

The Theorem applies in particular to the following examples.

Example 2.2.4. Let $\mathrm{S}$ be an algebraic space and $\mathrm{X}_{\bullet} \rightarrow \mathrm{S}$ a flat hypercover by algebraic spaces. We then obtain an augmented simplicial topos $\epsilon:\left(\mathrm{X}_{\bullet}\right.$, ét, $\mathscr{O}_{\mathrm{X}} \bullet$,ét $) \rightarrow\left(\mathrm{S}_{\text {ét }}, \mathscr{O}_{\text {ét }}\right)$. Note that this augmentation is flat. Let $\mathscr{C}$ denote the category of quasi-coherent sheaves on $\mathrm{S}_{\text {ét }}$. Then the category $\mathscr{C}_{\bullet}$ is the category of cartesian sheaves of $\mathscr{O}_{\mathrm{X}}$, ét $\mathrm{X}_{n}$ is quasi-coherent. Let $\mathrm{D}_{\mathrm{qcoh}}\left(\mathrm{X}_{\bullet}\right)$ denote the full subcategory of the derived category of $\mathscr{O}_{\mathrm{X}_{\bullet}, \text { ét }}-$ modules whose cohomology sheaves are quasi-coherent, and let $\mathrm{D}_{\mathrm{qcoh}}(\mathrm{S})$ denote the full subcategory of the derived category of $\mathscr{O}_{\text {Sét }}-$ modules whose cohomology sheaves are quasicoherent. Theorem 2.2.3 then shows that the pullback functor

$$
\epsilon^{*}: \mathrm{D}_{\mathrm{qcoh}}(\mathrm{S}) \rightarrow \mathrm{D}_{\mathrm{qcoh}}\left(\mathrm{X}_{\bullet}\right)
$$

is an equivalence of triangulated categories with quasi-inverse $\mathrm{R} \epsilon_{*}$.

Example 2.2.5. Let $\mathscr{X}$ be an algebraic stack and let $\mathrm{U} \bullet \rightarrow \mathscr{X}$ be a smooth hypercover by algebraic spaces. Let $\mathrm{D}(\mathscr{X})$ denote the derived category of sheaves of $\mathscr{O}_{\mathscr{X}_{\text {lis-ét }}}$-modules in the topos $\mathscr{X}_{\text {lis-ét }}$, and let $\mathrm{D}_{\mathrm{qcoh}}(\mathscr{X}) \subset \mathrm{D}(\mathscr{X})$ be the full subcategory of complexes with quasicoherent cohomology sheaves. 
Let $\mathrm{U}_{\bullet}^{+}$denote the strictly simplicial algebraic space obtained from $\mathrm{U}_{\bullet}$ by forgetting the degeneracies. Since the Lisse-Étale topos is functorial with respect to smooth morphisms, we therefore obtain a strictly simplicial topos $\mathrm{U}_{\bullet}$ lis-ét and a flat morphism of ringed topos

$$
\epsilon:\left(\mathrm{U}_{\bullet l i s-e ́ t}, \mathscr{O}_{\mathrm{U}_{\bullet l i s-e ́ t}}\right) \rightarrow\left(\mathscr{X}_{\text {lis-ét }}, \mathscr{O}_{\mathscr{X}_{\text {lis-ét }}}\right) .
$$

Then 2.2 .1 holds with $\mathscr{C}$ equal to the category of quasi-coherent sheaves on $\mathscr{X}$. The category

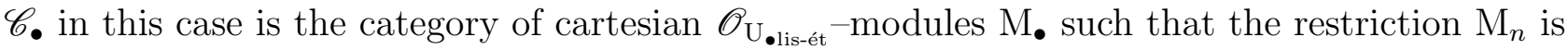
a quasi-coherent sheaf on $\mathrm{U}_{n}$ for all $n$. By 2.2.3 we then obtain an equivalence of triangulated categories

$$
\mathrm{D}_{\mathrm{qcoh}}(\mathscr{X}) \rightarrow \mathrm{D}_{\mathrm{qcoh}}\left(\mathrm{U}_{\bullet}, \text { lis-ét }\right),
$$

where the right side denotes the full subcategory of the derived category of $\mathscr{O}_{\mathrm{U}_{\bullet l i s-e ́ t}}$-modules with cohomology sheaves in $\mathscr{C}_{\bullet}$.

On the other hand, there is also a natural morphism of ringed topos

$$
\pi:\left(\mathrm{U}_{\bullet l i s-e ́ t}, \mathscr{O}_{\mathrm{U} \bullet \text { lis-ét }}\right) \rightarrow\left(\mathrm{U}_{\bullet e ́ t}, \mathscr{O}_{\mathrm{U}} \bullet e_{\mathrm{t}}\right)
$$

with $\pi_{*}$ and $\pi^{*}$ both exact functors. Let $\mathrm{D}_{\mathrm{qcoh}}\left(\mathrm{U}_{\bullet}\right.$ ét $)$ denote the full subcategory of the derived category of $\mathscr{O}_{\mathrm{U}}$ ét $^{-}$modules consisting of complexes whose cohomology sheaves are quasicoherent (i.e. cartesian and restrict to a quasi-coherent sheaf on each $\mathrm{U}_{\text {nét }}$ ). Then $\pi$ induces an equivalence of triangulated categories $\mathrm{D}_{\mathrm{qcoh}}\left(\mathrm{U}_{\bullet}\right.$ ét $) \simeq \mathrm{D}_{\mathrm{qcoh}}\left(\mathrm{U}_{\bullet}\right.$ lis-ét $)$. Putting it all together we obtain an equivalence of triangulated categories $\mathrm{D}_{\mathrm{qcoh}}\left(\mathscr{X}_{\text {lis-ét }}\right) \simeq \mathrm{D}_{\text {qcoh }}\left(\mathrm{U}_{\bullet \text { ét }}\right)$.

Example 2.2.6. Let $\mathscr{X}$ be an algebraic stack locally of finite type over $\mathrm{S}$ and $\mathscr{O}$ be a constant local Artinian ring $\Lambda$ of characteristic invertible on $\mathrm{S}$. Let $\mathrm{U}_{\bullet} \rightarrow \mathscr{X}$ be a smooth hypercover by algebraic spaces, and $\mathscr{T}$ • the localized topos $\left.\mathscr{X}_{\text {lis-ét }}\right|_{\text {U. }}$. Take $\mathscr{C}$ to be the category of constructible sheaves of $\mathscr{O}$-modules. Then 2.2 .3 gives an equivalence $\mathrm{D}_{c}\left(\mathscr{X}_{\text {lis-ét }}\right) \simeq \mathrm{D}_{c}\left(\mathscr{T}_{\bullet}, \Lambda\right)$. On the other hand, there is a natural morphism of topos $\lambda: \mathscr{T}_{\bullet} \rightarrow \mathrm{U}_{\bullet}$, et and one sees immediately that $\lambda_{*}$ and $\lambda^{*}$ induce an equivalence of derived categories $\mathrm{D}_{c}\left(\mathscr{T}_{\bullet}, \Lambda\right) \simeq \mathrm{D}_{c}\left(\mathrm{U}_{\bullet}, e t, \Lambda\right)$. It follows that $\mathrm{D}_{c}\left(\mathscr{X}_{\text {lis-ét }}\right) \simeq \mathrm{D}_{c}\left(\mathrm{U}_{\bullet}, e t\right)$.

2.3. The BBD gluing lemma. The purpose of this section is to explain how to modify the proof of the gluing lemma [7, 3.2.4] for unbounded complexes.

Let $\widetilde{\Delta}$ denote the strictly simplicial category of finite ordered sets with injective order preserving maps, and let $\Delta^{+} \subset \widetilde{\Delta}$ denote the full subcategory of nonempty finite ordered sets. For a morphism $\alpha$ in $\widetilde{\Delta}$ we write $s(\alpha)$ (resp. $b(\alpha))$ for its source (resp. target). 
Let $\mathrm{T}$ be a topos and $\mathrm{U}$. $\rightarrow e$ a strictly simplicial hypercovering of the initial object $e \in \mathrm{T}$. For $[n] \in \widetilde{\Delta}$ write $\mathrm{U}_{n}$ for the localized topos $\left.\mathrm{T}\right|_{\mathrm{U}_{n}}$ where by definition we set $\mathrm{U}_{\emptyset}=\mathrm{T}$. Then we obtain a strictly simplicial topos $\mathrm{U}$. with an augmentation $\pi: \mathrm{U}$. $\rightarrow \mathrm{T}$.

Let $\Lambda$ be a sheaf of rings in $\mathrm{T}$ and write also $\Lambda$ for the induced sheaf of rings in $\mathrm{U}$. so that $\pi$ is a morphism of ringed topos.

Let $\mathscr{C}$. denote a full substack of the fibered and cofibered category over $\widetilde{\Delta}$

$$
[n] \mapsto\left(\text { category of sheaves of } \Lambda \text {-modules in } \mathrm{U}_{n}\right)
$$

such that each $\mathscr{C}_{n}$ is a Serre subcategory of the category of $\Lambda$-modules in $\mathrm{U}_{n}$. For any $[n]$ we can then form the derived category $\mathrm{D}_{\mathscr{C}}\left(\mathrm{U}_{n}, \Lambda\right)$ of complexes of $\Lambda$-modules whose cohomology sheaves are in $\mathscr{C}_{n}$. The categories $\mathrm{D}_{\mathscr{C}}\left(\mathrm{U}_{n}, \Lambda\right)$ form a fibered and cofibered category over $\widetilde{\Delta}$.

We make the following assumptions on $\mathscr{C}$ :

Assumption 2.3.1. (i) For any $[n]$ the topos $\mathrm{U}_{n}$ is equivalent to the topos associated to a site $\mathscr{S}_{n}$ such that for any object $\mathrm{V} \in \mathscr{S}_{n}$ there exists an integer $n_{0}$ and a covering $\left\{\mathrm{V}_{j} \rightarrow \mathrm{V}\right\}$ in $\mathscr{S}_{n}$ such that for any $\mathrm{F} \in \mathscr{C}_{n}$ we have $\mathrm{H}^{n}\left(\mathrm{~V}_{j}, \mathrm{~F}\right)=0$ for all $n \geq n_{0}$.

(ii) The natural functor

$$
\mathscr{C}_{\emptyset} \rightarrow\left(\text { cartesian sections of }\left.\mathscr{C}\right|_{\Delta^{+}} \text {over } \Delta^{+}\right)
$$

is an equivalence of categories.

(iii) The category $\mathrm{D}(\mathrm{T}, \Lambda)$ is compactly generated.

Remark 2.3.2. The case we have in mind is when $\mathrm{T}$ is the lisse-étale topos of an algebraic stack $\mathscr{X}$ locally of finite type over an affine regular, noetherian scheme of dimension $\leq 1, \mathrm{U}$. is given by a hypercovering of $\mathscr{X}$ by schemes, $\Lambda$ is a Gorenstein local ring of dimension 0 and characteristic $l$ invertible on $\mathscr{X}$, and $\mathscr{C}$ is the category of constructible $\Lambda$-modules. In this case the category $\mathrm{D}_{c}\left(\mathscr{X}_{\text {lis-et }}, \Lambda\right)$ is compactly generated. Indeed a set of generators is given by sheaves $j_{!} \Lambda[i]$ for $i \in \mathbb{Z}$ and $j: \mathrm{U} \rightarrow \mathscr{X}$ an object of the lisse-étale site of $\mathscr{X}$.

There is also a natural functor

$$
\mathrm{D}_{\mathscr{C}}(\mathrm{T}, \Lambda) \rightarrow\left(\text { cartesian sections of }[n] \mapsto \mathrm{D}_{\mathscr{C}}\left(\mathrm{U}_{n}, \Lambda\right) \text { over } \Delta^{+}\right) .
$$

Theorem 2.3.3. Let $[n] \mapsto \mathrm{K}_{n} \in \mathrm{D}_{\mathscr{C}}\left(\mathrm{U}_{n}, \Lambda\right)$ be a cartesian section of $[n] \mapsto \mathrm{D}_{\mathscr{C}}\left(\mathrm{U}_{n}, \Lambda\right)$ over $\Delta^{+}$ such that $\mathscr{E} x t^{i}\left(\mathrm{~K}_{0}, \mathrm{~K}_{0}\right)=0$ for all $i<0$. Then $\left(\mathrm{K}_{n}\right)$ is induced by a unique object $\mathrm{K} \in \mathrm{D}_{\mathscr{C}}(\mathrm{T}, \Lambda)$ via the functor 2.3.2.1.

The uniqueness is the easy part: 
Lemma 2.3.4. Let $\mathrm{K}, \mathrm{L} \in \mathrm{D}(\mathrm{T}, \Lambda)$ and assume that $\mathscr{E} x t^{i}(\mathrm{~K}, \mathrm{~L})=0$ for $i<0$. Then $\mathrm{U} \mapsto$ $\operatorname{Hom}_{\mathrm{D}(\mathrm{U}, \Lambda)}\left(\left.\mathrm{K}\right|_{\mathrm{U}},\left.\mathrm{L}\right|_{\mathrm{U}}\right)$ is a sheaf.

Proof: Let $\mathscr{H}$ denote the complex $\mathscr{R} h o m(\mathrm{~K}, \mathrm{~L})$. By assumption the natural map $\mathscr{H} \rightarrow$ $\tau_{\geq 0} \mathscr{H}$ is an isomorphism. It follows that $\operatorname{Hom}_{\mathrm{D}(\mathrm{U}, \Lambda)}\left(\left.\mathrm{K}\right|_{\mathrm{U}},\left.\mathrm{L}\right|_{\mathrm{U}}\right)$ is equal to the value of $\mathscr{H}^{0}(\mathscr{H})$ on $\mathrm{U}$ which implies the lemma.

The existence part is more delicate. Let $\mathscr{A}$ denote the fibered and cofibered category over $\widetilde{\Delta}$ whose fiber over $[n] \in \widetilde{\Delta}$ is the category of $\Lambda$-modules in $\mathrm{U}_{n}$. For a morphism $\alpha:[n] \rightarrow[m]$, $\mathrm{F} \in \mathscr{A}(n)$ and $\mathrm{G} \in \mathscr{A}(m)$ we have

$$
\operatorname{Hom}_{\alpha}(\mathrm{F}, \mathrm{G})=\operatorname{Hom}_{\mathscr{A}(m)}\left(\alpha^{*} \mathrm{~F}, \mathrm{G}\right)=\operatorname{Hom}_{\mathscr{A}(n)}\left(\mathrm{F}, \alpha_{*} \mathrm{G}\right) .
$$

We write $\mathscr{A}^{+}$for the restriction of $\mathscr{A}$ to $\Delta^{+}$.

Define a new category tot $\left(\mathscr{A}^{+}\right)$as follows:

- The objects of tot $\left(\mathscr{A}^{+}\right)$are collections of objects $\left(\mathrm{A}^{n}\right)_{n \geq 0}$ with $\mathrm{A}^{n} \in \mathscr{A}(n)$.

- For two objects $\left(\mathrm{A}^{n}\right)$ and $\left(\mathrm{B}^{n}\right)$ we define

$$
\operatorname{Hom}_{\text {tot }\left(\mathscr{A}^{+}\right)}\left(\left(\mathrm{A}^{n}\right),\left(\mathrm{B}^{n}\right)\right):=\prod_{\alpha} \operatorname{Hom}_{\alpha}\left(\mathrm{A}^{s(\alpha)}, \mathrm{B}^{b(\alpha)}\right),
$$

where the product is taken over all morphisms in $\Delta^{+}$.

- If $f=\left(f_{\alpha}\right) \in \operatorname{Hom}\left(\left(\mathrm{A}^{n}\right),\left(\mathrm{B}^{n}\right)\right)$ and $g=\left(g_{\alpha}\right) \in \operatorname{Hom}\left(\left(\mathrm{B}^{n}\right),\left(\mathrm{C}^{n}\right)\right)$ are two morphisms then the composite is defined to be the collection of morphisms whose $\alpha$ component is defined to be

$$
(g \circ f)_{\alpha}:=\sum_{\alpha=\beta \gamma} g_{\beta} f_{\gamma}
$$

where the sum is taken over all factorizations of $\alpha$.

The category tot $\left(\mathscr{A}^{+}\right)$is an additive category.

Let $(\mathrm{K}, d)$ be a complex in $\operatorname{tot}\left(\mathscr{A}^{+}\right)$so for every degree $n$ we are given a family of objects $\left(\mathrm{K}^{n}\right)^{m} \in \mathscr{A}(m)$. Set

$$
\mathrm{K}^{n, m}:=\left(\mathrm{K}^{n+m}\right)^{n} .
$$

For $\alpha:[n] \rightarrow[m]$ in $\Delta^{+}$let $d(\alpha)$ denote the $\alpha$-component of $d$ so

$$
d(\alpha) \in \operatorname{Hom}_{\alpha}\left(\left(\mathrm{K}^{p}\right)^{n},\left(\mathrm{~K}^{p+1}\right)^{m}\right)=\operatorname{Hom}_{\alpha}\left(\mathrm{K}^{n, p-n}, \mathrm{~K}^{m, m-p-1}\right)
$$

or equivalently $d(\alpha)$ is a map $\mathrm{K}^{n, p} \rightarrow \mathrm{K}^{m, p+n-m+1}$. In particular, $d\left(\mathrm{id}_{[n]}\right)$ defines a map $\mathrm{K}^{n, m} \rightarrow$ $\mathrm{K}^{n, m+1}$ and as explained in [7, 3.2.8] this map makes $\mathrm{K}^{n, *}$ a complex. Furthermore for any $\alpha$ the map $d(\alpha)$ defines an $\alpha$-map of complexes $\mathrm{K}^{n, *} \rightarrow \mathrm{K}^{m, *}$ of degree $n-m+1$. The collection of complexes $\mathrm{K}^{n, *}$ can also be defined as follows. For an integer $p$ let $\mathrm{L}^{p} \mathrm{~K}$ denote the subcomplex 
with $\left(\mathrm{L}^{p} \mathrm{~K}\right)^{n, m}$ equal to 0 if $n<p$ and $\mathrm{K}^{n, m}$ otherwise. Note that for any $\alpha:[n] \rightarrow[m]$ which is not the identity map $[n] \rightarrow[n]$ the image of $d(\alpha)$ is contained in $\mathrm{L}^{p+1} \mathrm{~K}$. Taking the associated graded of $\mathrm{L}$ we see that

$$
\operatorname{gr}_{\mathrm{L}}^{n} \mathrm{~K}[n]=\left(\mathrm{K}^{n, *}, d^{\prime \prime}\right)
$$

where $d^{\prime \prime}$ denote the differential $(-1)^{n} d\left(\operatorname{id}_{[n]}\right)$. Note that the functor $(\mathrm{K}, d) \mapsto \mathrm{K}^{n, *}$ commutes with the formation of cones and with shifting of degrees.

As explained in [7, 3.2.8] a complex in $\operatorname{tot}\left(\mathscr{A}^{+}\right)$is completely characterized by the data of a complex $\mathrm{K}^{n, *} \in \mathrm{C}\left(\mathscr{A}^{+}\right)$for every $[n] \in \Delta^{+}$and for every morphism $\alpha:[n] \rightarrow[m]$ an $\alpha$-morphism $d(\alpha): \mathrm{K}^{n, *} \rightarrow \mathrm{K}^{m, *}$ of degree $n-m+1$, such that $d\left(\operatorname{id}_{[n]}\right)$ is equal to $(-1)^{n}$ times the differential of $\mathrm{K}^{n, *}$ and such that for every $\alpha$ we have

$$
\sum_{\alpha=\beta \gamma} d(\beta) d(\gamma)=0
$$

Via this dictionary, a morphism $f: \mathrm{K} \rightarrow \mathrm{L}$ in $\mathrm{C}\left(\operatorname{tot}\left(\mathscr{A}^{+}\right)\right)$is given by an $\alpha-$ map $f(\alpha): \mathrm{K}^{n * *} \rightarrow$ $\mathrm{K}^{m, *}$ of degree $n-m$ for every morphism $\alpha:[n] \rightarrow[m]$ in $\Delta^{+}$such that for any morphism $\alpha$ we have

$$
\sum_{\alpha=\beta \gamma} d(\beta) f(\gamma)=\sum_{\alpha=\beta \gamma} f(\beta) d(\gamma) .
$$

Let $\mathrm{K}\left(\operatorname{tot}\left(\mathscr{A}^{+}\right)\right)$denote the category whose objects are complexes in $\operatorname{tot}\left(\mathscr{A}^{+}\right)$and whose morphisms are homotopy classes of morphisms of complexes. The category $\mathrm{K}\left(\operatorname{tot}\left(\mathscr{A}^{+}\right)\right)$is a triangulated category. Let $\mathrm{L} \subset \mathrm{K}\left(\operatorname{tot}\left(\mathscr{A}^{+}\right)\right)$denote the full subcategory of objects $\mathrm{K}$ for which each $\mathrm{K}^{n, *}$ is acyclic for all $n$. The category $\mathrm{L}$ is a localizing subcategory of $\mathrm{K}\left(\operatorname{tot}\left(\mathscr{A}^{+}\right)\right)$in the sense of [8, 1.3] and hence the localized category $\mathrm{D}\left(\operatorname{tot}\left(\mathscr{A}^{+}\right)\right)$exists. The category $\mathrm{D}\left(\operatorname{tot}\left(\mathscr{A}^{+}\right)\right)$ is obtained from $\mathrm{K}\left(\operatorname{tot}\left(\mathscr{A}^{+}\right)\right)$by inverting quasi-isomorphisms. Recall that an object $\mathrm{K} \in$ $\mathrm{K}\left(\operatorname{tot}\left(\mathscr{A}^{+}\right)\right)$is called $\mathrm{L}$-local if for any object $\mathrm{X} \in \mathrm{L}$ we have $\operatorname{Hom}_{\mathrm{K}(\operatorname{tot}(\mathscr{A}+))}(\mathrm{X}, \mathrm{K})=0$. Note that the functor $\mathrm{K} \mapsto \mathrm{K}^{n \text {,* }}$ descends to a functor

$$
\mathrm{D}\left(\operatorname{tot}\left(\mathscr{A}^{+}\right)\right) \rightarrow \mathrm{D}\left(\mathrm{U}_{n}, \Lambda\right)
$$

We define $\mathrm{D}^{+}\left(\operatorname{tot}\left(\mathscr{A}^{+}\right)\right) \subset \mathrm{D}\left(\operatorname{tot}\left(\mathscr{A}^{+}\right)\right)$to be the full subcategory of objects $\mathrm{K}$ for which there exists an integer $\mathrm{N}$ such that $\mathscr{H}^{j}\left(\mathrm{~K}^{n, *}\right)=0$ for all $n$ and all $j \leq \mathrm{N}$.

Recall [8, 4.3] that a localization for an object $\mathrm{K} \in \mathrm{K}\left(\operatorname{tot}\left(\mathscr{A}^{+}\right)\right)$is a morphism $\mathrm{K} \rightarrow \mathrm{I}$ with I an L-local object such that for any L-local object $\mathrm{Z}$ the natural map

$$
\operatorname{Hom}_{\mathrm{K}\left(\operatorname{tot}\left(\mathscr{A}^{+}\right)\right)}(\mathrm{I}, \mathrm{Z}) \rightarrow \operatorname{Hom}_{\mathrm{K}\left(\operatorname{tot}\left(\mathscr{A}^{+}\right)\right)}(\mathrm{K}, \mathrm{Z})
$$

is an isomorphism. 
Lemma 2.3.5. A morphism $\mathrm{K} \rightarrow \mathrm{I}$ is a localization if and only if $\mathrm{I}$ is $\mathrm{L}$-local and for every $n$ the map $\mathrm{K}^{n, *} \rightarrow \mathrm{I}^{n, *}$ is a quasi-isomorphism.

Proof: By [8, 2.9] the morphism 2.3.4.1 can be identified with the natural map

$$
\operatorname{Hom}_{\mathrm{D}\left(\operatorname{tot}\left(\mathscr{A}^{+}\right)\right)}(\mathrm{I}, \mathrm{Z}) \rightarrow \operatorname{Hom}_{\mathrm{D}\left(\operatorname{tot}\left(\mathscr{A}^{+}\right)\right)}(\mathrm{K}, \mathrm{Z}) .
$$

If $\mathrm{K} \rightarrow \mathrm{I}$ is a localization it follows that this map is a bijection for every L-local Z. By Yoneda's lemma applied to the full subcategory of $\mathrm{D}\left(\operatorname{tot}\left(\mathscr{A}^{+}\right)\right)$of objects which can be represented by $\mathrm{L}$-local objects, it follows that this holds if and only if $\mathrm{K} \rightarrow \mathrm{I}$ induces an isomorphism in $\mathrm{D}\left(\operatorname{tot}\left(\mathscr{A}^{+}\right)\right)$which is the assertion of the lemma.

Proposition 2.3.6. Let $\mathrm{K} \in \mathrm{C}\left(\operatorname{tot}\left(\mathscr{A}^{+}\right)\right)$be an object with each $\mathrm{K}^{n, *}$ homotopically injective. Then $\mathrm{K}$ is $\mathrm{L}$-local.

Proof: Let $\mathrm{X} \in \mathrm{L}$ be an object. We have to show that any morphism $f: \mathrm{X} \rightarrow \mathrm{K}$ in $\mathrm{C}\left(\operatorname{tot}\left(\mathscr{A}^{+}\right)\right)$is homotopic to zero. Such a homotopy $h$ is given by a collection of maps $h(\alpha)$ such that

$$
f(\alpha)=-\sum_{\alpha=\beta \gamma} d(\beta) h(\gamma)+h(\beta) d(\gamma)
$$

We usually write just $h$ for $h\left(\operatorname{id}_{[n]}\right)$.

We construct these maps $h(\alpha)$ by induction on $b(\alpha)-s(\alpha)$. For $s(\alpha)=b(\alpha)$ we choose the $h(\alpha)$ to be any homotopies between the maps $f\left(\operatorname{id}_{[n]}\right)$ and the zero maps.

For the inductive step, it suffices to show that

$$
\Psi(\alpha)=f(\alpha)+d(\alpha) h+h d(\alpha)+\sum_{\alpha=\beta \gamma}{ }^{\prime} d(\beta) h(\gamma)+h(\beta) d(\gamma)
$$

commutes with the differentials $d$, where $\Sigma_{\alpha=\beta \gamma}^{\prime}$ denotes the sum over all possible factorizations with $\beta$ and $\gamma$ not equal to the identity maps. For then $\Psi(\alpha)$ is homotopic to zero and we can take $h(\alpha)$ to be a homotopy between $\Psi(\alpha)$ and 0 .

Define

$$
\mathrm{A}(\alpha)=\sum_{\alpha=\beta \gamma}{ }^{\prime} d(\beta) h(\gamma)+h(\gamma) d(\beta)
$$

and

$$
\mathrm{B}(\alpha)=d(\alpha) h+h d(\alpha)+\mathrm{A}(\alpha) .
$$

\section{Lemma 2.3.7.}

$$
\sum_{\alpha=\beta \gamma}{ }^{\prime} \mathrm{A}(\beta) d(\gamma)-d(\beta) \mathrm{A}(\gamma)=\sum_{\alpha=\beta \gamma}{ }^{\prime} h(\beta) \mathrm{S}(\gamma)-\mathrm{S}(\beta) h(\gamma)
$$


where $\mathrm{S}(\alpha)$ denotes $\sum_{\alpha=\beta \gamma}{ }^{\prime} d(\beta) d(\gamma)$.

Proof:

$$
\begin{aligned}
\sum_{\alpha=\beta \gamma}{ }^{\prime} \mathrm{A}(\beta) d(\gamma)-d(\beta) \mathrm{A}(\gamma) & =\sum_{\alpha=\epsilon \rho \gamma}{ }^{\prime} d(\epsilon) h(\rho) d(\gamma)+h(\epsilon) d(\rho) d(\gamma)-d(\epsilon) h(\rho) d(\gamma)-d(\epsilon) d(\rho) h(\gamma) \\
& =\sum_{\alpha=\beta \gamma}{ }^{\prime} h(\beta) \mathrm{S}(\gamma)-\mathrm{S}(\beta) h(\gamma)
\end{aligned}
$$

where $\Sigma_{\alpha=\epsilon \rho \gamma}^{\prime}$ denotes the sum over all possible factorizations with $\epsilon, \rho$, and $\gamma$ not equal to the identity maps.

\section{Lemma 2.3.8.}

$\sum_{\alpha=\beta \gamma}{ }^{\prime} \mathrm{B}(\beta) d(\gamma)-d(\beta) \mathrm{B}(\gamma)=-h(d(\alpha) d+d d(\alpha))+(d(\alpha) d+d d(\alpha)) h+\sum_{\alpha=\beta \gamma}{ }^{\prime} h(\beta) \mathrm{S}(\gamma)-\mathrm{S}(\beta) h(\gamma)$

Proof:

$$
\begin{aligned}
& \sum_{\alpha=\beta \gamma}{ }^{\prime} \mathrm{B}(\beta) d(\gamma)-d(\beta) \mathrm{B}(\gamma) \\
= & \sum_{\alpha=\beta \gamma}{ }^{\prime} d(\beta) h d(\gamma)+h d(\beta) d(\gamma)+\mathrm{A}(\beta) d(\gamma)-d(\beta) d(\gamma) h-d(\beta) h d(\gamma)-d(\beta) \mathrm{A}(\gamma) \\
= & -h(d(\alpha) d+d d(\alpha))+(d(\alpha) d+d d(\alpha)) h+\sum_{\alpha=\beta \gamma} ' h(\beta) \mathrm{S}(\gamma)-\mathrm{S}(\beta) h(\gamma) .
\end{aligned}
$$

We can now prove 2.3.6. We compute

$$
\begin{aligned}
& d \mathrm{~A}(\alpha)-\mathrm{A}(\alpha) d \\
= & \sum_{\alpha=\beta \gamma}{ }^{\prime} d d(\beta) h(\gamma)+d h(\beta) d(\gamma)-d(\beta) h(\gamma) d-h(\beta) d(\gamma) d \\
= & \sum_{\alpha=\beta \gamma}{ }^{\prime} d d(\beta) h(\gamma)+(-f(\beta)-\mathrm{B}(\beta)-h(\beta) d) d(\gamma)-d(\beta)(-f(\gamma)-\mathrm{B}(\gamma)-d h(\gamma))-h(\beta) d(\gamma) d \\
= & \sum_{\alpha=\beta \gamma}{ }^{\prime} d d(\beta) h(\gamma)-f(\beta) d(\gamma)-\mathrm{B}(\beta) d(\gamma) \\
- & h(\beta) d d(\gamma)+d(\beta) f(\gamma)+d(\beta) \mathrm{B}(\gamma)+d(\beta) d h(\gamma)-h(\beta) d(\gamma) d \\
= & {\left[\sum_{\alpha=\beta \gamma} '(-\mathrm{S}(\beta) h(\gamma))+h(\beta) \mathrm{S}(\gamma)-f(\beta) d(\gamma)+d(\beta) f(\gamma)\right] } \\
+ & h(d(\alpha) d+d d(\alpha))-(d(\alpha) d+d d(\alpha)) h-\sum_{\alpha=\beta \gamma}{ }^{\prime} h(\beta) \mathrm{S}(\gamma)-\mathrm{S}(\beta) h(\gamma) \\
= & f(\alpha) d-d f(\alpha)+f d(\alpha)-d(\alpha) f+h(d(\alpha) d+d d(\alpha))-(d(\alpha) d+d d(\alpha)) h .
\end{aligned}
$$


So finally

$$
\begin{aligned}
& d \Psi(\alpha)-\Psi(\alpha) d \\
= & d f(\alpha)+d d(\alpha) h+d h d(\alpha)+d \mathrm{~A}(\alpha)-f(\alpha) d-d(\alpha) h d-h d(\alpha) d-\mathrm{A}(\alpha) d \\
= & d f(\alpha)+d d(\alpha) h+d h d(\alpha)-f(\alpha) d-d(\alpha) h d-h d(\alpha) d \\
+ & f(\alpha) d-d f(\alpha)+f d(\alpha)-d(\alpha) f+h d(\alpha) d+h d d(\alpha)-d(\alpha) d h-d d(\alpha) h \\
= & 0 .
\end{aligned}
$$

This completes the proof of 2.3.6.

Let

$$
\epsilon^{*}: \mathrm{C}(\mathscr{A}(\emptyset)) \rightarrow \mathrm{C}\left(\operatorname{tot}\left(\mathscr{A}^{+}\right)\right)
$$

be the functor sending a complex $\mathrm{K}$ to the object of $\mathrm{C}\left(\operatorname{tot}\left(\mathscr{A}^{+}\right)\right)$with $\epsilon^{*} \mathrm{~K}^{n \text {,* }}=\mathrm{K}$ with maps $d\left(\operatorname{id}_{[n]}\right)$ equal to $(-1)^{n}$ times the differential, for $\partial_{i}:[n] \rightarrow[n+1]$ the map $d\left(\partial_{i}\right)$ is the canonical map of complexes, and all other $d(\alpha)$ 's are zero. The functor $\epsilon^{*}$ takes quasi-isomorphisms to quasi-isomorphisms and hence induces a functor

$$
\epsilon^{*}: \mathrm{D}(\mathscr{A}(\emptyset)) \rightarrow \mathrm{D}\left(\operatorname{tot}\left(\mathscr{A}^{+}\right)\right)
$$

Lemma 2.3.9. The functor $\epsilon^{*}$ has a right adjoint $\mathrm{R} \epsilon_{*}: \mathrm{D}\left(\operatorname{tot}\left(\mathscr{A}^{+}\right)\right) \rightarrow \mathrm{D}(\mathscr{A}(\emptyset))$ and $\operatorname{R} \epsilon_{*}$ is a triangulated functor.

Proof: We apply the adjoint functor theorem [18, 4.1]. By our assumptions the category $\mathrm{D}(\mathscr{A}(\emptyset))$ is compactly generated. Therefore it suffices to show that $\epsilon^{*}$ commutes with coproducts (direct sums) which is immediate.

More concretely, the functor $\mathrm{R} \epsilon_{*}$ can be computed as follows. If $\mathrm{K}$ is $\mathrm{L}$-local and there exists an integer $\mathrm{N}$ such that for every $n$ we have $\mathrm{K}^{n, m}=0$ for $m<\mathrm{N}$, then $\mathrm{R} \epsilon_{*} \mathrm{~K}$ is represented by the complex with

$$
\left(\epsilon_{*} \mathrm{~K}\right)^{p}=\oplus_{n+m=p} \epsilon_{n *} \mathrm{~K}^{n, m}
$$

with differential given by $\sum d(\alpha)$. This follows from Yoneda's lemma and the observation that for any $\mathrm{F} \in \mathrm{D}(\mathscr{A}(\emptyset))$ we have

$$
\begin{aligned}
\operatorname{Hom}_{\mathrm{D}(\mathscr{A}(\emptyset))}\left(\mathrm{F}, \mathrm{R} \epsilon_{*} \mathrm{~K}\right) & =\operatorname{Hom}_{\mathrm{D}\left(\operatorname{tot}\left(\mathscr{A}^{+}\right)\right)}\left(\epsilon^{*} \mathrm{~F}, \mathrm{~K}\right) \\
& =\operatorname{Hom}_{\mathrm{K}\left(\operatorname{tot}\left(\mathscr{A}^{+}\right)\right)}\left(\epsilon^{*} \mathrm{~F}, \mathrm{~K}\right) \quad \text { since } \mathrm{K} \text { is L-local } \\
& =\operatorname{Hom}_{\mathrm{K}(\mathscr{A}(\emptyset))}\left(\mathrm{F}, \epsilon_{*} \mathrm{~K}\right) \text { by [7, 3.2.12]. }
\end{aligned}
$$

Lemma 2.3.10. For any $\mathrm{F} \in \mathrm{D}^{+}(\mathscr{A}(\emptyset))$ the natural map $\mathrm{F} \rightarrow \mathrm{R} \epsilon_{*} \epsilon^{*} \mathrm{~F}$ is an isomorphism. 
Proof: Represent F by a complex of injectives. Then $\epsilon^{*} \mathrm{~F}$ is $\mathrm{L}-$ local by 2.3.6, The result then follows from cohomological descent.

Proposition 2.3.11. Let $\mathrm{K} \in \mathrm{D}_{\text {cart }}^{+}\left(\operatorname{tot}\left(\mathscr{A}^{+}\right)\right)$be an object. Then $\epsilon^{*} \mathrm{R} \epsilon_{*} \mathrm{~K} \rightarrow \mathrm{K}$ is an isomorphism. In particular, $\mathrm{R} \epsilon_{*}$ and $\epsilon^{*}$ induce an equivalence of categories between $\mathrm{D}_{\text {cart }}^{+}\left(\operatorname{tot}\left(\mathscr{A}^{+}\right)\right)$ and $\mathrm{D}^{+}(\mathscr{A}(\emptyset))$.

Proof: For any integer $s$ and system $\left(\mathrm{K}^{n, *}, d(\alpha)\right)$ defining an object of $\mathrm{C}\left(\operatorname{tot}\left(\mathscr{A}^{+}\right)\right)$we obtain a new object by $\left(\tau_{\leq s} \mathrm{~K}^{n, *}, d(\alpha)\right)$ since for any $\alpha$ which is not the identity morphism the map $d(\alpha)$ has degree $\leq 0$. We therefore obtain a functor $\tau_{\leq s}: \mathrm{C}\left(\operatorname{tot}\left(\mathscr{A}^{+}\right)\right) \rightarrow \mathrm{C}\left(\operatorname{tot}\left(\mathscr{A}^{+}\right)\right)$ which takes quasi-isomorphisms to quasi-isomorphisms and hence descends to a functor

$$
\tau_{\leq s}: \mathrm{D}\left(\operatorname{tot}\left(\mathscr{A}^{+}\right)\right) \rightarrow \mathrm{D}\left(\operatorname{tot}\left(\mathscr{A}^{+}\right)\right)
$$

Furthermore, there is a natural morphism of functors $\tau_{\leq s} \rightarrow \tau_{\leq s+1}$ and we have

$$
\mathrm{K} \simeq \operatorname{hocolim} \tau_{\leq s} \mathrm{~K}
$$

Note that the functor $\epsilon^{*}$ commutes with homotopy colimits since it commutes with direct sums. If we show the proposition for the $\tau_{\leq s} \mathrm{~K}$ then we see that the natural map

$$
\epsilon^{*}\left(\operatorname{hocolimR} \epsilon_{*} \tau_{\leq s} \mathrm{~K}\right) \simeq \operatorname{hocolim} \epsilon^{*} \mathrm{R} \epsilon_{*} \tau_{\leq s} \mathrm{~K} \rightarrow \operatorname{hocolim} \tau_{\leq s} \mathrm{~K} \simeq \mathrm{K}
$$

is an isomorphism. In particular $\mathrm{K}$ is in the essential image of $\epsilon^{*}$. Write $\mathrm{K}=\epsilon^{*} \mathrm{~F}$. Then by 2.3.10 $\mathrm{R} \epsilon_{*} \mathrm{~K} \simeq \mathrm{F}$ whence $\epsilon^{*} \mathrm{R} \epsilon_{*} \mathrm{~K} \rightarrow \mathrm{K}$ is an isomorphism.

It therefore suffices to prove the proposition for $\mathrm{K}$ bounded above. Considering the distinguished triangles associated to the truncations $\tau_{\leq s} \mathrm{~K}$ we further reduce to the case when $\mathrm{K}$ is concentrated in just a single degree. In this case, $\mathrm{K}$ is obtained by pullback from an object of $\mathscr{A}(\emptyset)$ and the proposition again follows from 2.3.10.

For an object $\mathrm{K} \in \mathrm{K}\left(\operatorname{tot}\left(\mathscr{A}^{+}\right)\right)$, we define $\tau_{\geq s} \mathrm{~K}$ to be the cone of the natural map $\tau_{\leq s-1} \mathrm{~K} \rightarrow$ $\mathrm{K}$.

Observe that the category $\mathrm{K}\left(\operatorname{tot}\left(\mathscr{A}^{+}\right)\right)$has products and therefore also homotopy limits. Let $\mathrm{K} \in \mathrm{K}_{\mathscr{C}}\left(\operatorname{tot}\left(\mathscr{A}^{+}\right)\right)$be an object. By 2.3.11, for each $s$ we can find a bounded below complex of injectives $\mathrm{I}_{s} \in \mathrm{C}(\mathscr{A}(\emptyset))$ and a quasi-isomorphism $\sigma_{s}: \tau_{\geq s} \mathrm{~K} \rightarrow \epsilon^{*} \mathrm{I}_{s}$. Since $\epsilon^{*} \mathrm{I}_{s}$ is $\mathrm{L}-$ local and $\epsilon^{*}: \mathrm{D}^{+}(\mathscr{A}(\emptyset)) \rightarrow \mathrm{D}\left(\operatorname{tot}\left(\mathscr{A}^{+}\right)\right)$is fully faithful by 2.3 .11 , the maps $\tau_{\geq s-1} \mathrm{~K} \rightarrow \tau_{\geq s} \mathrm{~K}$ induce a 
unique morphism $t_{s}: \mathrm{I}_{s-1} \rightarrow \mathrm{I}_{s}$ in $\mathrm{K}(\mathscr{A}(\emptyset))$ such that the diagrams

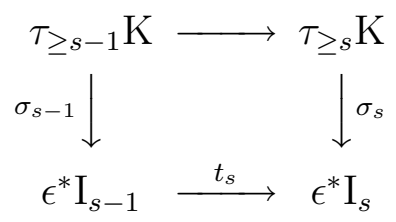

commutes in $\mathrm{K}\left(\operatorname{tot}\left(\mathscr{A}^{+}\right)\right)$.

Proposition 2.3.12. The natural map $\mathrm{K} \rightarrow$ holim $\epsilon^{*} \mathrm{I}_{s}$ is a quasi-isomorphism.

Proof: It suffices to show that for all $n$ the map $\mathrm{K}^{n, *} \rightarrow$ holim $\epsilon_{n}^{*} \mathrm{I}_{s}$ is a quasi-isomorphism, where $\epsilon_{n}: \mathrm{U}_{n} \rightarrow \mathrm{T}$ is the projection. Let $\mathscr{S}_{n}$ be a site inducing $\mathrm{U}_{n}$ as in 2.3.1. We show that for any integer $i$ the map of presheaves on the subcategory of $\mathscr{S}_{n}$ satisfying the finiteness assumption in 2.3.1 (i)

$$
\left(\mathrm{V} \rightarrow \mathrm{U}_{n}\right) \mapsto \mathrm{H}^{i}\left(\mathrm{~V}, \mathrm{~K}^{n, *}\right) \rightarrow \mathrm{H}^{i}\left(\mathrm{~V}, \operatorname{holim} \epsilon_{n}^{*} \mathrm{I}_{s}\right)
$$

is an isomorphism. For this note that for every $s$ there is a distinguished triangle

$$
\mathscr{H}^{s}\left(\mathrm{~K}^{n, *}\right)[s] \rightarrow \epsilon_{n}^{*} \mathrm{I}_{s} \rightarrow \epsilon_{n}^{*} \mathrm{I}_{s-1}
$$

and hence by the assumption 2.3.1 (i) the map

$$
\mathrm{H}^{i}\left(\mathrm{~V}, \epsilon_{n}^{*} \mathrm{I}_{s}\right) \rightarrow \mathrm{H}^{i}\left(\mathrm{~V}, \epsilon_{n}^{*} \mathrm{I}_{s-1}\right)
$$

is an isomorphism for $s<i-n_{0}$. Since each $\epsilon_{n}^{*} \mathrm{I}_{s}$ is a complex of injectives, the complex $\prod_{s} \epsilon_{n}^{*} \mathrm{I}_{s}$ is also a complex of injectives. Therefore

$$
\mathrm{H}^{i}\left(\mathrm{~V}, \prod_{s} \epsilon_{n}^{*} \mathrm{I}_{s}\right)=\mathrm{H}^{i}\left(\prod_{s} \epsilon_{n}^{*} \mathrm{I}_{s}(\mathrm{~V})\right)=\prod_{s} \mathrm{H}^{i}\left(\epsilon_{n}^{*} \mathrm{I}_{s}(\mathrm{~V})\right)
$$

It follows that there is a canonical long exact sequence

$$
\cdots \longrightarrow \prod_{s} \mathrm{H}^{i}\left(\epsilon_{n}^{*} \mathrm{I}_{s}(\mathrm{~V})\right) \stackrel{1-\text { shift }}{\longrightarrow} \prod_{s} \mathrm{H}^{i}\left(\epsilon_{n}^{*} \mathrm{I}_{s}(\mathrm{~V})\right) \longrightarrow \mathrm{H}^{i}\left(\mathrm{~V}, \text { holim } \epsilon_{n}^{*} \mathrm{I}_{s}\right) \longrightarrow \cdots
$$

From this and the fact that the maps 2.3.12.1 are isomorphisms for $s$ sufficiently big it follows that the cohomology group $\mathrm{H}^{i}\left(\mathrm{~V}\right.$, holim $\left.\epsilon_{n}^{*} \mathrm{I}_{s}\right)$ is isomorphic to $\mathrm{H}^{i}\left(\mathrm{~V}, \mathrm{~K}^{n, *}\right)$ via the canonical map. Passing to the associated sheaves we obtain the proposition.

Corollary 2.3.13. Every object $\mathrm{K} \in \mathrm{D}_{\mathscr{C}}\left(\operatorname{tot}\left(\mathscr{A}^{+}\right)\right)$is in the essential image of the functor

$$
\epsilon^{*}: \mathrm{D}_{\mathscr{C}}(\mathscr{A}(\emptyset)) \rightarrow \mathrm{D}_{\mathscr{C}}\left(\operatorname{tot}\left(\mathscr{A}^{+}\right)\right)
$$


Proof: Since $\epsilon^{*}$ also commutes with products and hence also homotopy limits we find that $\mathrm{K} \simeq \epsilon^{*}\left(\right.$ holim $\left.\mathrm{I}_{s}\right)$ in $\mathrm{D}_{\mathscr{C}}\left(\operatorname{tot}\left(\mathscr{A}^{+}\right)\right)$(note that $\mathscr{H}^{i}($ holimI $s)$ is in $\mathscr{C}$ since this can be checked after applying $\epsilon^{*}$ ).

Lemma 2.3.14. Let $[n] \mapsto \mathrm{K}^{n}$ be a cartesian section of $[n] \mapsto \mathrm{D}\left(\mathrm{U}_{n}, \Lambda\right)$ such that $\mathscr{E} x t^{i}\left(\mathrm{~K}^{n}, \mathrm{~K}^{n}\right)=$ 0 for all $i<0$. Then $\left(\mathrm{K}^{n}\right)$ is induced by an object of $\mathrm{D}\left(\operatorname{tot}\left(\mathscr{A}^{+}\right)\right)$.

Proof: Represent each $\mathrm{K}^{n}$ by a homotopically injective complex (denoted by the same letter) in $\mathrm{C}\left(\mathrm{U}_{n}, \Lambda\right)$ for every $n$. For each morphism $\partial_{i}:[n] \rightarrow[n+1]$ (the unique morphism whose image does not contain $i$ ) choose a $\partial_{i}$-map of complexes $\partial_{i}^{*}: \mathrm{K}^{n} \rightarrow \mathrm{K}^{n+1}$ inducing the given map in $\mathrm{D}\left(\mathrm{U}_{n+1}, \Lambda\right)$ by the strictly simplicial structure. The proof then proceeds by the same argument used to prove [7, 3.2.9].

Combining this with 2.3 .13 we obtain 2.3 .3 ,

\section{Dualizing COMPleX}

3.1. Dualizing complexes on algebraic spaces. Let $\mathrm{W}$ be an algebraic space and $w: \mathrm{W} \rightarrow \mathrm{S}$ be a separated ${ }^{5}$ morphism of finite type with $\mathrm{W}$ an algebraic space. We'll define $\Omega_{w}$ by glueing as follows. By the comparison lemma ([2], III.4.1), the étale topos $\mathrm{W}_{\text {ét }}$ can be defined using the site Étale(W) whose objects are étale morphisms $\mathrm{A}: \mathrm{U} \rightarrow \mathrm{W}$ where $a: \mathrm{U} \rightarrow \mathrm{S}$ is affine of finite type. The localized topos $\mathrm{W}_{\text {ét|U }}$ coincides with $\mathrm{U}_{\text {ét }}$.

Unless otherwise explicitly stated, we will ring the various étale or lisse-étale topos which will be appear by the constant Gorenstein ring $\Lambda$ of dimension 0 of the introduction.

Notice that this is not true for the corresponding lisse-étale topos. This fact will cause some difficulties below. Let $\Omega$ denote the dualizing complex of $\mathrm{S}$, and let $\alpha: \mathrm{U} \rightarrow \mathrm{S}$ denote the structural morphism. We define

$$
\Omega_{\mathrm{A}}=\alpha ! \Omega \in \mathrm{D}\left(\mathrm{U}_{\text {ét }}, \Lambda\right)=\mathrm{D}\left(\mathrm{W}_{\text {ét } \mid \mathrm{U}}\right) .
$$

which is the (relative) dualizing complex of $\mathrm{U}$, and therefore one gets by biduality ([9], «Th. finitude» 4.3)

$$
\mathscr{R h o m}\left(\Omega_{\mathrm{A}}, \Omega_{\mathrm{A}}\right)=\Lambda
$$

implying at once

$$
\mathscr{E} x t_{\mathrm{W}_{\mathrm{e} t \mid \mathrm{U}}}^{i}\left(\Omega_{\mathrm{A}}, \Omega_{\mathrm{A}}\right)=0 \text { if } i<0 .
$$

\footnotetext{
${ }^{5}$ Probably one can assume only that $w$ quasi-separated, cf. 4, XVII.7; but we do not need this more general version.
} 
We want to apply the glueing theorem 2.3.3. Let us therefore consider a diagram

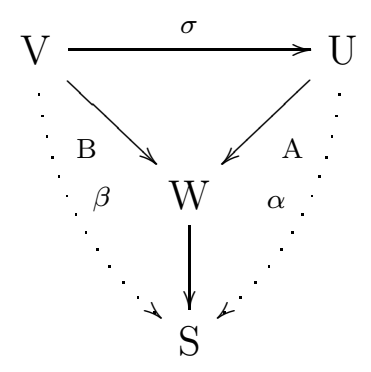

with a commutative triangle and $\mathrm{A}, \mathrm{B} \in$ Étale(W).

Lemma 3.1.1. There is a functorial isomorphism

$$
\sigma^{*} \Omega_{\mathrm{A}}=\Omega_{\mathrm{B}}
$$

Proof: Let $\tilde{\mathrm{W}}=\mathrm{U} \times_{\mathrm{W}} \mathrm{V}$ : it is an affine scheme, of finite type over $\mathrm{S}$, and étale over both $\mathrm{U}, \mathrm{V}$. In fact, we have a cartesian diagram

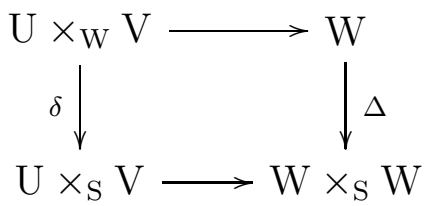

where $\Delta$ is a closed immersion (W/S separated) showing that $\tilde{W}=\mathrm{U} \times{ }_{\mathrm{W}} \mathrm{V}$ is a closed subscheme of $\mathrm{U} \times{ }_{\mathrm{S}} \mathrm{V}$ which is affine. Looking at the graph diagram with cartesian square

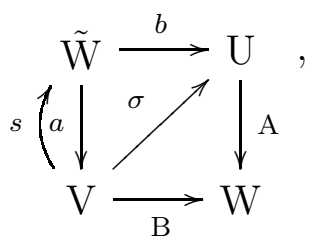

we get that $a, b$ are étale and separated like A, B. One deduces a commutative diagram

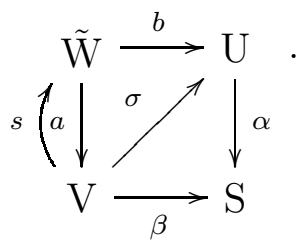

We claim that

$$
b^{*} \alpha{ }^{\prime} \Omega=a^{*} \beta^{!} \Omega .
$$


Indeed, $a, b$ being smooth of relative dimension 0 , one has

$$
b^{*} \alpha ! \Omega=b^{!} \alpha ! \Omega
$$

and analogously

$$
a^{*} \beta ! \Omega=a^{!} \beta^{!} \Omega
$$

Because $\alpha b=\beta a$, one gets $b^{!} \alpha^{!}=a^{!} \beta^{!}$. Pulling back by $s$ gives the result.

Therefore $\left(\Omega_{\mathrm{A}}\right)_{\mathrm{A} \in \text { Étale(W) }}$ defines locally an object $\Omega_{w}$ of $\mathrm{D}(\mathrm{W})$ with vanishing negative $\mathscr{E} x t$ 's (recall that $w: \mathrm{W} \rightarrow \mathrm{S}$ is the structural morphism). By 2.3.3, we get

Proposition 3.1.2. There exists a unique $\Omega_{w} \in \mathrm{D}\left(\mathrm{W}_{\text {ét }}\right)$ such that $\Omega_{w \mid \mathrm{U}}=\Omega_{\mathrm{A}}$.

We need functoriality for smooth morphisms.

Lemma 3.1.3. If $f: \mathrm{W}_{1} \rightarrow \mathrm{W}_{2}$ is a smooth $\mathrm{S}$-morphism of relative dimension $d$ between algebraic space separated and of finite type over $\mathrm{S}$ with dualizing complexes $\Omega_{1}, \Omega_{2}$, then

$$
f^{*} \Omega_{2}=\Omega_{1}\langle-d\rangle
$$

Proof: Start with $\mathrm{U}_{2} \rightarrow \mathrm{W}_{2}$ étale and surjective with $\mathrm{U}_{2}$ affine say. Then, $\tilde{\mathrm{W}}_{1}=\mathrm{W}_{1} \times_{\mathrm{U}_{2}} \mathrm{~W}_{2}$ is an algebraic space separated and of finite type over $S$. Let $U_{1} \rightarrow \tilde{W}_{1}$ be a surjective étale morphism with $\mathrm{U}_{1}$ affine and let $g: \mathrm{U}_{1} \rightarrow \mathrm{U}_{2}$ be the composition $\mathrm{U}_{1} \rightarrow \tilde{\mathrm{W}}_{1} \rightarrow \mathrm{U}_{2}$. It is a smooth morphism of relative dimension $d$ between affine schemes of finite type from which follows the formula $g^{!}(-)=g^{*}(-)\langle d\rangle$. Therefore, the pull-backs of $\mathrm{L}_{1}=\Omega_{1}\langle-d\rangle$ and $f^{*} \Omega_{2}$ on $\mathrm{U}_{1}$ are the same, namely $\Omega_{\mathrm{U}_{1}}$. One deduces that these complexes coincide on the covering sieve $W_{\text {lét } \mid U_{1}}$ and therefore coincide by 2.3 .4 (because the relevant negative $\mathscr{E} x t^{i}$ s sanish.

3.2. Étale dualizing data. Let $\mathscr{X} \rightarrow \mathrm{S}$ be an algebraic S-stack locally of finite type. Let $\mathrm{A}: \mathrm{U} \rightarrow \mathscr{X}$ in Lisse-Et $(\mathscr{X})$ and $\alpha: \mathrm{U} \rightarrow \mathrm{S}$ the composition $\mathrm{U} \rightarrow \mathscr{X} \rightarrow \mathrm{S}$. We define

$$
\mathrm{K}_{\mathrm{A}}=\Omega_{\alpha}\left\langle-d_{\mathrm{A}}\right\rangle \in \mathrm{D}_{c}\left(\mathrm{U}_{\text {ét }}, \Lambda\right)
$$

where $d_{\mathrm{A}}$ is the relative dimension of $\mathrm{A}$ (which is locally constant). Up to shift and Tate torsion, $\mathrm{K}_{\mathrm{A}}$ is the (relative) dualizing complex of $\mathrm{U}$ and therefore one gets by biduality

$$
\mathscr{R h o m}\left(\mathrm{K}_{\mathrm{A}}, \mathrm{K}_{\mathrm{A}}\right)=\Lambda \text { and } \mathscr{E} x t_{\mathrm{U}_{\text {ét }}}^{i}\left(\mathrm{~K}_{\mathrm{A}}, \mathrm{K}_{\mathrm{A}}\right)=0 \text { if } i<0 \text {. }
$$


We need again a functoriality property of $\mathrm{K}_{\mathrm{A}}$. Let us consider a diagram

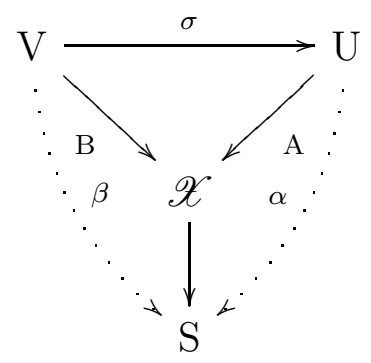

with a 2-commutative triangle and $\mathrm{A}, \mathrm{B} \in \operatorname{Lisse-} \operatorname{Et}(\mathscr{X})$.

Lemma 3.2.1. There is a functorial identification

$$
\sigma^{*} \mathrm{~K}_{\mathrm{A}}=\mathrm{K}_{\mathrm{B}} .
$$

Proof: Let $\mathrm{W}=\mathrm{U} \times \mathscr{X} \mathrm{V}$ which is an algebraic space. One has a commutative diagram with cartesian square

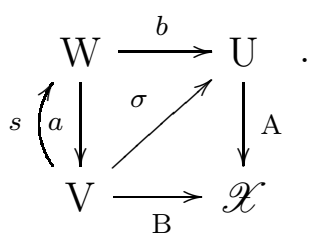

In particular, $a, b$ are smooth and separated like A, B. One deduces a commutative diagram

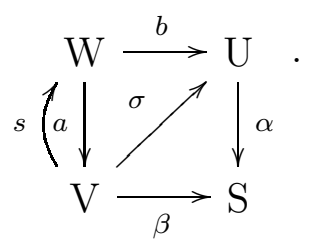

I claim that

$$
b^{*} \mathrm{~K}_{\mathrm{A}}=a^{*} \mathrm{~K}_{\mathrm{B}}=\mathrm{K}_{w} .
$$

where $w$ denotes the structural morphism $\mathrm{W} \rightarrow \mathrm{S}$.

Indeed, $a, b$ being smooth of relative dimensions $d_{\mathrm{A}}, d_{\mathrm{B}}$, one has 3.1 .3

$$
b^{*} \mathrm{~K}_{\mathrm{A}}=b^{*} \Omega_{\alpha}\left\langle-d_{\mathrm{A}}\right\rangle=\Omega_{\alpha}\left\langle-d_{\mathrm{A}}-d_{\mathrm{B}}\right\rangle
$$

and analogously

$$
a^{*} K_{\mathrm{B}}=a^{*} \Omega\left\langle-d_{\mathrm{B}}\right\rangle=\Omega_{\alpha}\left\langle-d_{\mathrm{B}}-d_{\mathrm{A}}\right\rangle .
$$

Because $\alpha b=\beta a$, one gets $b^{!} \alpha^{!}=a^{!} \beta^{!}$. Pulling back by $s$ gives the result. 
Remark 3.2.2. Because all S-schemes of finite type satisfy $\operatorname{cd}_{\Lambda}(\mathrm{X})<\infty$, we know that $\mathrm{K}_{\mathrm{X}}$ is not only of finite quasi-injective dimension but of finite injective dimension ([5], I.1.5). By construction this implies that $\mathrm{K}_{\mathrm{A}}$ is of finite injective dimension for $\mathrm{A}$ as above.

3.3. Lisse-étale dualizing data. In order to define $\Omega_{\mathscr{X}} \in \mathrm{D}\left(\mathscr{X}_{\text {lis-ét }}\right)$ by glueing, we need glueing data $\kappa_{\mathrm{A}} \in \mathrm{D}\left(\mathscr{X}_{\text {lis-ét|U }}\right), \mathrm{U} \in \operatorname{Lisse-Et}(\mathscr{X})$. The inclusion

$$
\text { Étale }(\mathrm{U}) \hookrightarrow \operatorname{Lisse-Et}(\mathscr{X})_{\mid \mathrm{U}}
$$

induces a continuous morphism of sites. Since finite inverse limits exist in Étale(U) and this morphism of sites preserves such limits, it defines by $([2,4.9 .2)$ a morphism of topos (we abuse notation slightly and omit the dependence on A from the notation)

$$
\epsilon: \mathscr{X}_{\text {lis-ét } \mid \mathrm{U}} \rightarrow \mathrm{U}_{\text {ét }} \text {. }
$$

3.3.1. Let us describe more explicitely the morphism $\epsilon$. Let Lisse-Et $(\mathscr{X})_{\mid \mathrm{U}}$ denote the category of morphisms $\mathrm{V} \rightarrow \mathrm{U}$ in $\operatorname{Lisse-Et}(\mathscr{X})$. The category $\operatorname{Lisse-Et}(\mathscr{X})_{\mid \mathrm{U}}$ has a Grothendieck topology induced by the topology on Lisse-Et $(\mathscr{X})$, and the resulting topos is canonicallly isomorphic to the localized topos $\mathscr{X}_{\text {lis-ét|U }}$. Note that there is a natural inclusion Lisse-Et(U) $\hookrightarrow$ $\operatorname{Lisse-Et}(\mathscr{X})_{\mid \mathrm{U}}$ but this is not an equivalence of categories since for an object $(\mathrm{V} \rightarrow \mathrm{U}) \in$ $\operatorname{Lisse-Et}(\mathscr{X})_{\mid \mathrm{U}}$ the morphism $\mathrm{V} \rightarrow \mathrm{U}$ need not be smooth. It follows that an element of $\mathscr{X}_{\text {lis-ét|U }}$ is equivalent to giving for every $\mathrm{U}$-scheme of finite type $\mathrm{V} \rightarrow \mathrm{U}$, such that the composite $\mathrm{V} \rightarrow \mathrm{U} \rightarrow \mathscr{X}$ is smooth, a sheaf $\mathscr{F}_{\mathrm{V}} \in \mathrm{V}_{\text {ét }}$ together with morphisms $f^{-1} \mathscr{F}_{\mathrm{V}} \rightarrow \mathscr{F}_{\mathrm{V}^{\prime}}$ for U-morphisms $f: \mathrm{V}^{\prime} \rightarrow \mathrm{V}$. Furthermore, these morphisms satisfy the usual compatibility with compositions. Viewing $\mathscr{X}_{\text {lis-ét|U }}$ in this way, the functor $\epsilon^{-1}$ maps $\mathscr{F}$ on $\mathrm{U}_{\text {ét }}$ to $\mathscr{F}_{\mathrm{V}}=\pi^{-1} \mathscr{F} \in \mathrm{V}_{\text {ét }}$

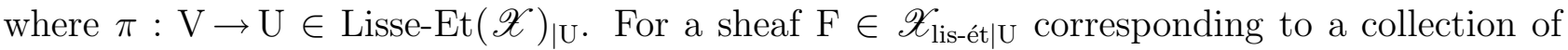
sheaves $\mathscr{F}_{\mathrm{V}}$, the sheaf $\epsilon_{*} \mathrm{~F}$ is simply the sheaf $\mathscr{F}_{\mathrm{U}}$.

In particular, the functor $\epsilon_{*}$ is exact and, accordingly, that $\mathrm{H}^{*}(\mathrm{U}, \mathrm{F})=\mathrm{H}^{*}\left(\mathrm{U}_{\text {ét }}, \mathrm{F}_{\mathrm{U}}\right)$ for any shaf of $\Lambda$ modules of $\mathscr{X}$.

3.3.2. A morphism $f: \mathrm{U} \rightarrow \mathrm{V}$ of $\operatorname{Lisse-Et}(\mathscr{X})$ induces a diagram

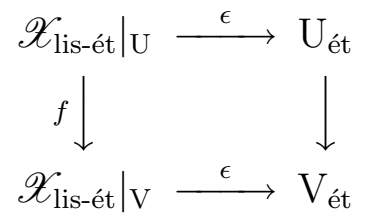

where $\mathscr{X}_{\text {lis-ét|U }} \rightarrow \mathscr{X}_{\text {lis-ét|V }}$ is the localization morphism ([2, IV.5.5.2) which we still denote by $f$ slightly abusively. For a sheaf $\mathscr{F} \in \mathrm{V}_{\text {ét }}$, the pullback $f^{-1} \epsilon^{-1} \mathscr{F}$ is the sheaf corresponding to 
the system which to any $p: \mathrm{U}^{\prime} \rightarrow \mathrm{U}$ associates $p^{-1} f^{-1} \mathscr{F}$. In particular, $f^{-1} \circ \epsilon^{-1}=\epsilon^{-1} \circ f^{-1}$ which implies that 3.3 .2 .1 is a commutative diagram of topos. We define

$$
\kappa_{\mathrm{A}}=\epsilon^{*} \mathrm{~K}_{\mathrm{A}} \in \mathrm{D}\left(\mathscr{X}_{\text {lis-ét } \mid \mathrm{U}}\right) \text {. }
$$

By the preceding discussion, if

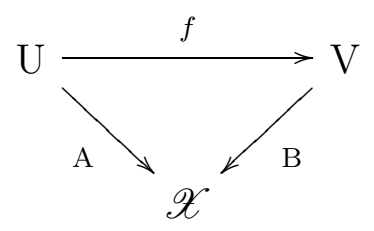

is a morphism in Lisse-Et $(\mathscr{X})$, we get

$$
f^{*} \kappa_{\mathrm{B}}=\kappa_{\mathrm{A}}
$$

showing that the family $\left(\kappa_{\mathrm{A}}\right)$ defines locally an object of $\mathrm{D}\left(\mathscr{X}_{\text {lis-ét }}\right)$.

3.4. Glueing the local dualizing data. Let $\mathrm{A} \in \operatorname{Lisse-\operatorname {Et}}(\mathscr{X})$ and $\epsilon: \mathscr{X}_{\text {lis-ét } \mid \mathrm{U}} \rightarrow \mathrm{U}_{\text {ét }}$ be as above. We need first the vanishing of $\mathscr{E} x t^{i}\left(\kappa_{\mathrm{A}}, \kappa_{\mathrm{A}}\right), i<0$.

Lemma 3.4.1. Let $\mathscr{F}, \mathscr{G} \in \mathrm{D}\left(\mathrm{U}_{\text {ét }}\right)$. One has

(i) $\operatorname{Ext}^{i}\left(\epsilon^{*} \mathscr{F}, \epsilon^{*} \mathscr{G}\right)=\operatorname{Ext}^{i}(\mathscr{F}, \mathscr{G})$.

(ii) The étale sheaf $\mathscr{E} x t^{i}\left(\epsilon^{*} \mathscr{F}, \epsilon^{*} \mathscr{G}\right)_{\mathrm{U}}$ on $\mathrm{U}_{\text {ét }}$ is $\mathscr{E} x t_{\mathrm{U}_{\text {ét }}}^{i}(\mathscr{F}, \mathscr{G})$.

Proof: Since $\epsilon_{*}$ is exact and for any sheaf $\mathrm{F} \in \mathrm{U}_{\text {ét }}$ one has $\mathrm{F}=\epsilon_{*} \epsilon^{*} \mathrm{~F}$, the adjunction map $\mathrm{F} \rightarrow \mathrm{R} \epsilon_{*} \epsilon^{*} \mathrm{~F}$ is an isomorphism for any $\mathrm{F} \in \mathrm{D}\left(\mathrm{U}_{\text {ét }}\right)$. By trivial duality, one gets

$$
\epsilon_{*} \mathscr{R h o m}\left(\epsilon^{*} \mathscr{F}, \epsilon^{*} \mathscr{G}\right)=\mathscr{R h o m}\left(\mathscr{F}, \epsilon_{*} \epsilon^{*} \mathscr{G}\right)=\mathscr{R h o m}(\mathscr{F}, \mathscr{G}) \text {. }
$$

Taking $\mathscr{H}^{i} \mathrm{R} \Gamma$ gives (i).

By construction, $\mathscr{E} x t^{i}\left(\epsilon^{*} \mathscr{F}, \epsilon^{*} \mathscr{F}\right)_{\mathrm{U}}$ is the sheaf associated to the presheaf on $\mathrm{U}_{\text {ét }}$ which to any étale morphism $\pi: \mathrm{V} \rightarrow \mathrm{U}$ associates $\operatorname{Ext}^{i}\left(\pi^{*} \epsilon^{*} \mathscr{F}, \pi^{*} \epsilon^{*} \mathscr{G}\right)$ where $\pi^{*}$ is the the pull-back functor associated to the localization morphism

$$
\left(\left.\mathscr{X}_{\text {lis-ét }}\right|_{\mathrm{U}}\right)_{\mid \mathrm{V}}=\mathscr{X}_{\text {lis-ét } \mid \mathrm{V}} \rightarrow \mathscr{X}_{\text {lis-ét } \mid \mathrm{U}}
$$

([3], V.6.1). By the commutativity of the diagram 3.3.2.1, one has $\pi^{*} \epsilon^{*}=\epsilon^{*} \pi^{*}$. Therefore

$$
\operatorname{Ext}^{i}\left(\pi^{*} \epsilon^{*} \mathscr{F}, \pi^{*} \epsilon^{*} \mathscr{G}\right)=\operatorname{Ext}^{i}\left(\epsilon^{*} \pi^{*} \mathscr{F}, \epsilon^{*} \pi^{*} \mathscr{G}\right)=\operatorname{Ext}_{\mathrm{V}_{\text {ét }}^{i}}^{i}\left(\pi^{*} \mathscr{F}, \pi^{*} \mathscr{G}\right),
$$

the last equality is by $(i)$. Since $\mathscr{E} x t_{\mathrm{U}_{\text {ét }}}(\mathscr{F}, \mathscr{G})$ is also the sheaf associated to this presheaf we obtain (ii).

Using 3.2.0.2, one obtains 
Corollary 3.4.2. One has $\mathscr{R} h o m\left(\kappa_{\mathrm{A}}, \kappa_{\mathrm{A}}\right)=\Lambda$ and therefore $\mathscr{E} x t^{i}\left(\kappa_{\mathrm{A}}, \kappa_{\mathrm{A}}\right)=0$ if $i<0$.

The discussion above shows that we can apply 2.3 .3 to $\left(\kappa_{\mathrm{A}}\right)$ to get

Proposition 3.4.3. There exists $\Omega_{\mathscr{X}}(p) \in \mathrm{D}^{b}\left(\mathscr{X}_{\text {lis-ét }}\right)$ inducing $\kappa_{\mathrm{A}}$ for all $\mathrm{A} \in \operatorname{Lisse}-\operatorname{Et}(\mathscr{X})_{\mid \mathrm{X}}$. It is well defined up to unique isomorphism.

The independence of the presentation is straightforward and is left to the reader :

Lemma 3.4.4. Let $p_{i}: \mathrm{X}_{i} \rightarrow \mathscr{X}, i=1,2$ two presentations as above. There exists a canonical, functorial isomorphism $\Omega_{\mathscr{X}}\left(p_{1}\right) \stackrel{\sim}{\rightarrow} \Omega_{\mathscr{X}}\left(p_{2}\right)$.

Definition 3.4.5. The dualizing complex of $\mathscr{X}$ is the "essential" value $\Omega_{\mathscr{X}} \in \mathrm{D}^{b}\left(\mathscr{X}_{\text {lis-ét }}\right)$ of $\Omega_{\mathscr{X}}(p)$, where $p$ runs over presentations of $\mathscr{X}$. It is well defined up to canonical functorial

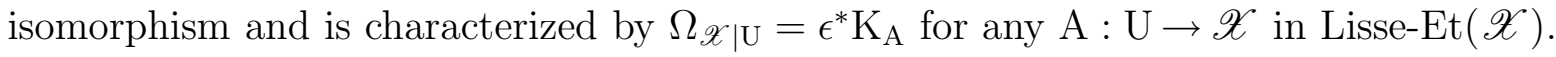

3.5. Biduality. For A, B any abelian complexes of some topos, there is a biduality morphism

$$
\mathrm{A} \rightarrow \mathscr{R h o m}(\mathscr{R h o m}(\mathrm{A}, \mathrm{B}), \mathrm{B})
$$

(replace B by some homotopically injective complex isomorphic to it in the derived catgory).

In general, it is certainly not an isomorphism.

Lemma 3.5.1. Let $u: \mathrm{U} \rightarrow \mathrm{S}$ be a separated $\mathrm{S}$-scheme (or algebraic space) of finite type and $\mathrm{A} \in \mathrm{D}_{c}\left(\mathrm{U}_{\text {ét }}, \Lambda\right)$. Then the biduality morphism

$$
\mathrm{A} \rightarrow \mathscr{R h o m}\left(\mathscr{R h o m}\left(\mathrm{A}, \mathrm{K}_{\mathrm{U}}\right), \mathrm{K}_{\mathrm{U}}\right)
$$

is an isomorphism (where $\mathrm{K}_{\mathrm{U}}$ is -up to shift and twist- the dualizing complex of $\mathrm{U}_{\text {ét }}$ ).

Proof: If A is moreover bounded, it is the usual theorem of [9]. Let us denote by $\tau_{n}$ the two-sides truncation functor

$$
\tau_{\geq-n} \tau_{\leq n}
$$

We know that $\mathrm{K}_{\mathrm{U}}$ is a dualizing complex ([5], exp. I), and is of finite injective dimension (3.2.2); the homology in degree $n$ of the biduality morphism $\mathrm{A} \rightarrow \mathrm{DD}(\mathrm{A})$ is therefore the same as the homology in degree $n$ of the biduality morphism $\tau_{m} \mathrm{~A} \rightarrow \mathrm{DD}\left(\tau_{m} \mathrm{~A}\right)$ for $m$ large enough and the lemma follows. 
We will be interested in a commutative diagram

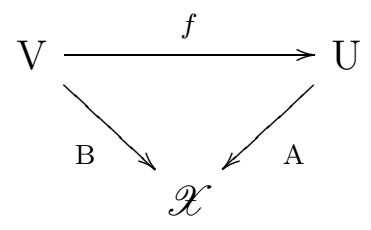

as above.

Lemma 3.5.2. Let $\mathscr{F} \in \mathrm{D}_{c}\left(\mathscr{X}_{\text {lis-ét }}\right)$ and let $\mathscr{F}_{\mathrm{U}} \in \mathrm{D}_{c}\left(\mathrm{U}_{\text {ét }}\right)$ be the object obtained by restriction.

(i) One has f* $\mathscr{R h o m}\left(\mathscr{F}_{\mathrm{U}}, \mathrm{K}_{\mathrm{A}}\right)=\mathscr{R h o m}\left(f^{*} \mathscr{F}_{\mathrm{U}}, f^{*} \mathrm{~K}_{\mathrm{A}}\right)=\mathscr{R h o m}\left(f^{*} \mathscr{F}_{\mathrm{U}}, \mathrm{K}_{\mathrm{B}}\right)$.

(ii) Moreover, $\mathscr{R} h o m\left(\mathscr{F}_{\mathrm{U}}, \mathrm{K}_{\mathrm{A}}\right)$ is constructible.

Proof: Let's prove $(i)$. By 3.2.1, one has $f^{*} \mathrm{~K}_{\mathrm{A}}=\mathrm{K}_{\mathrm{B}}$, therefore one has a morphism

$$
f^{*} \mathscr{R h o m}\left(\mathscr{F}_{\mathrm{U}}, \mathrm{K}_{\mathrm{A}}\right) \rightarrow \mathscr{R h o m}\left(f^{*} \mathscr{F}_{\mathrm{U}}, \mathrm{K}_{\mathrm{B}}\right) .
$$

To prove that it is an isomorphism, consider first the case when $f$ is smooth. Because both $\mathrm{K}_{\mathrm{A}}$ and $\mathrm{K}_{\mathrm{B}}$ are of finite injective dimension (3.2.2), one can assume that $\mathrm{F}$ is bounded where it is obviously true by reduction to F the constant sheaf (or use [5], I.7.2). Therefore the result holds when $f$ is smooth.

From the case of a smooth morphism, one reduces the proof in general to the case when $\mathscr{X}$ is a scheme. Let $\mathscr{F}_{\mathscr{X}} \in \mathrm{D}_{c}\left(\mathscr{X}_{\text {et }}\right)$ denote the complex obtained by restricting $\mathscr{F}$. By the smooth case already considered, we have

$$
\begin{aligned}
f^{*} \mathscr{R h o m}\left(\mathscr{F}_{\mathrm{U}}, \mathrm{K}_{\mathrm{A}}\right) & \simeq f^{*} \mathrm{~A}^{*} \mathscr{R h o m}\left(\mathscr{F}_{\mathscr{X}}, \mathrm{K}_{\mathscr{X}}\right) \\
& =\mathrm{B}^{*} \mathscr{R h o m}\left(\mathscr{F}_{\mathscr{X}}, \mathrm{K}_{\mathscr{X}}\right) \\
& \simeq \mathscr{R h o m}\left(\mathrm{B}^{*} \mathscr{F}_{\mathrm{X}}, \mathrm{B}^{*} \mathrm{~K}_{\mathscr{X}}\right) \\
& \simeq \mathscr{R h o m}\left(f^{*} \mathscr{F}_{\mathrm{U}}, f^{*} \mathrm{~K}_{\mathrm{A}}\right) .
\end{aligned}
$$

For $(i i)$, one can also assume $\mathscr{F}$ bounded and one uses [5], I.7.1.

Lemma 3.5.3. Let $\mathscr{F} \in \mathrm{D}_{c}\left(\mathscr{X}_{\text {lis-ét }}\right)$. Then,

$$
\epsilon^{*} \mathscr{R h o m}_{\mathrm{U}_{\text {ét }}}\left(\mathscr{F} \mathrm{U}, \mathrm{K}_{\mathrm{A}}\right)=\mathscr{R h o m}\left(\mathscr{F}, \Omega_{\mathscr{X}}\right)_{\mid \mathrm{U}}
$$

where $\mathscr{F}_{\mathrm{U}}=\epsilon_{*} \mathscr{F}_{\mid \mathrm{U}}$ is the restriction of $\mathscr{F}$ to Étale $(\mathrm{U})$.

Proof: By definition of constructibility, $\mathscr{H}^{i}(\mathscr{F})$ are cartesian sheaves. In other words, $\epsilon_{*}$ being exact, the adjunction morphism

$$
\epsilon^{*} \mathscr{F}_{\mathrm{U}}=\epsilon^{*} \epsilon_{*} \mathscr{F}_{\mid \mathrm{U}} \rightarrow \mathscr{F}_{\mid \mathrm{U}}
$$


is an isomorphism. We therefore have

$$
\begin{aligned}
\mathscr{R h o m}(\mathscr{F}, \Omega)_{\mid \mathrm{U}} & =\mathscr{R h o m}\left(\mathscr{F}_{\mid \mathrm{U}}, \Omega_{\mid \mathrm{U}}\right) \\
& =\mathscr{R h o m}\left(\epsilon^{*} \mathscr{F}_{\mathrm{U}}, \epsilon^{*} \mathrm{~K}_{\mathrm{A}}\right)
\end{aligned}
$$

Therefore, we get a morphism

$$
\epsilon^{*} \mathscr{R} h o m_{\mathrm{U}_{\mathrm{et}}}\left(\mathscr{F}_{\mathrm{U}}, \mathrm{K}_{\mathrm{A}}\right) \rightarrow \mathscr{R h o m}\left(\epsilon^{*} \mathscr{F}_{\mathrm{U}}, \epsilon^{*} \mathrm{~K}_{\mathrm{A}}\right)=\mathscr{R} \operatorname{hom}\left(\mathscr{F}, \Omega_{\mathscr{X}}\right)_{\mid \mathrm{U}} .
$$

By 3.4.1, one has

$$
\mathscr{E} x t^{i}\left(\epsilon^{*} \mathscr{F}_{\mathrm{U}}, \epsilon^{*} \mathrm{~K}_{\mathrm{A}}\right)_{\mathrm{V}}=\mathscr{E} x t_{\mathrm{V}_{\text {ét }}^{i}}^{i}\left(f^{*} \mathscr{F}_{\mathrm{U}}, f^{*} \mathrm{~K}_{\mathrm{A}}\right)
$$

But, one has

$$
\left.\mathscr{H}^{i}\left(\epsilon^{*} \mathscr{R} h o m_{\mathrm{U}_{\hat{e t}}}\left(\mathscr{F}_{\mathrm{U}}, \mathrm{K}_{\mathrm{A}}\right)\right)_{\mathrm{V}}=f^{*} \mathscr{E} x t_{\mathrm{U}_{\mathrm{ett}}}^{i}\left(\mathscr{F}_{\mathrm{U}}, \mathrm{K}_{\mathrm{A}}\right)\right)
$$

and the lemma follows from 3.5 .2 .

One gets immediately (cf. [5], I.1.4)

Corollary 3.5.4. $\Omega_{\mathscr{X}}$ is of finite quasi-injective dimension.

Remark 3.5.5. It seems over-optimistic to think that $\Omega_{\mathscr{X}}$ would be of finite injective dimension even if $\mathscr{X}$ is a scheme.

Lemma 3.5.6. If $\mathrm{A} \in \mathrm{D}_{c}(\mathscr{X})$, then $\mathscr{R} h o m\left(\mathrm{~A}, \Omega_{\mathscr{X}}\right) \in \mathrm{D}_{c}(\mathscr{X})$.

Proof: Immediate consequence of 3.5 .2 and 3.5 .3 .

Corollary 3.5.7. The (contravariant) functor

$$
\mathrm{D}_{\mathscr{X}}:\left\{\begin{array}{clc}
\mathrm{D}_{c}(\mathscr{X}) & \rightarrow \mathrm{D}_{c}(\mathscr{X}) \\
\mathscr{F} & \mapsto \mathscr{R} h o m\left(\mathscr{F}, \Omega_{\mathscr{X}}\right)
\end{array}\right.
$$

is an involution. More precisely, the morphism

$$
\iota: \mathrm{Id} \rightarrow \mathrm{D}_{\mathscr{X}} \circ \mathrm{D}_{\mathscr{X}}
$$

induced by 3.5.0.1 is an isomorphism.

Proof: We have to prove that the cone $\mathrm{C}$ of the biduality morphism is zero in the derived category, that is to say

$$
\mathrm{C}_{\mathrm{U}}=\epsilon_{*} \mathrm{C}_{\mid \mathrm{U}}=0 \text { in } \mathrm{D}_{c}\left(\mathrm{U}_{\text {ét }}\right) \text {. }
$$


But we have

$$
\begin{aligned}
& \epsilon_{*}\left(\mathscr{R h o m}\left(\mathscr{R h o m}\left(\mathscr{F}, \Omega_{\mathscr{X}}\right), \Omega_{\mathscr{X}}\right)\right)_{\mid \mathrm{U}}=\epsilon_{*} \mathscr{R h o m}\left(\mathscr{R h o m}\left(\mathscr{F}, \Omega_{\mathscr{X}}\right)_{\mid \mathrm{U}}, \Omega_{\mathscr{X} \mid \mathrm{U}}\right) \\
& \text { 3.5.3 } \epsilon_{*} \mathscr{R h o m}\left(\epsilon^{*} \mathscr{R h o m}\left(\mathscr{F}_{\mathrm{U}}, \mathrm{K}_{\mathrm{A}}\right), \Omega_{\mathscr{X} \mid \mathrm{U}}\right) \\
& =\mathscr{R h o m}\left(\mathscr{R} h o m\left(\mathscr{F}_{\mathrm{U}}, \mathrm{K}_{\mathrm{A}}\right), \epsilon_{*} \epsilon^{*} \mathrm{~K}_{\mathrm{A}}\right) \text { by trivial duality } \\
& =\mathscr{R h o m}\left(\mathscr{R h o m}\left(\mathscr{F}_{\mathrm{U}}, \mathrm{K}_{\mathrm{A}}\right), \mathrm{K}_{\mathrm{A}}\right) \\
& \text { 3.5.1 } \mathscr{F}_{\mathrm{U}}
\end{aligned}
$$

Remark 3.5.8. Verdier duality $\mathrm{D}_{\mathscr{X}}$ identifies $\mathrm{D}_{c}^{a}$ and $\mathrm{D}_{c}^{-a}$ with $a=\varnothing, \pm 1, b$ and the usual conventions $-\varnothing=\varnothing$ and $-b=b$.

Proposition 3.5.9. One has a canonical (bifunctorial) morphism

$$
\mathscr{R h o m}(\mathrm{A}, \mathrm{B})=\mathscr{R h o m}(\mathrm{D}(\mathrm{B}), \mathrm{D}(\mathrm{A}))
$$

for all $\mathrm{A}, \mathrm{B} \in \mathrm{D}_{c}(\mathscr{X})$.

Proof: Let us prove first a well-known formula

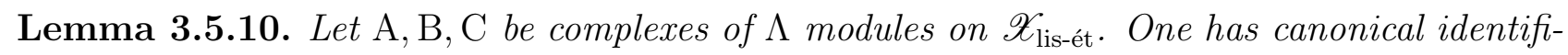
cations

$$
\mathscr{R h o m}(\mathrm{A}, \mathscr{R h o m}(\mathrm{B}, \mathrm{C}))=\mathscr{R h o m}(\mathrm{A} \stackrel{\mathrm{L}}{\otimes} \mathrm{B}, \mathrm{C})=\mathscr{R h o m}(\mathrm{B}, \mathscr{R h o m}(\mathrm{A}, \mathrm{C})) \text {. }
$$

Proof: One can assume A, B homotopically flat and C homotopically injective. Let X be an acyclic complex. One has

$$
\operatorname{Hom}(\mathrm{X}, \mathscr{H} \mathrm{om}(\mathrm{B}, \mathrm{C}))=\operatorname{Hom}(\mathrm{X} \otimes \mathrm{B}, \mathrm{C})
$$

Because $\mathrm{B}$ est homotopically flat, $\mathrm{X} \otimes \mathrm{B}$ is acyclic. Moreover, $\mathrm{C}$ being homotopically injective, the abelian complex $\operatorname{Hom}(\mathrm{X} \otimes \mathrm{B}, \mathrm{C})$ is acyclic. Therefore, $\mathscr{H} \mathrm{om}(\mathrm{B}, \mathrm{C})$ homotopically injective. One gets therefore

$$
\mathscr{R h o m}(\mathrm{A}, \mathscr{R h o m}(\mathrm{B}, \mathrm{C}))=\mathscr{H} \operatorname{om}(\mathrm{A}, \mathscr{H} \operatorname{om}(\mathrm{B}, \mathrm{C}))=\mathscr{H} \operatorname{om}(\mathrm{A} \otimes \mathrm{B}, \mathrm{C})=\mathscr{R h o m}(\mathrm{A} \stackrel{\mathrm{L}}{\otimes} \mathrm{B}, \mathrm{C}) .
$$

One gets then

$$
\mathscr{R h o m}(\mathrm{D}(\mathrm{B}), \mathrm{D}(\mathrm{A}))=\mathscr{R h o m}\left(\mathrm{D}(\mathrm{B}), \mathscr{R} h o m\left(\mathrm{~A}, \Omega_{\mathscr{X}}\right)\right) \stackrel{\text { 3.5.10 }}{=} \mathscr{R} h o m(\mathrm{~A}, \mathrm{D} \circ \mathrm{D}(\mathrm{B}))=\mathscr{R h o m}(\mathrm{A}, \mathrm{B}) \text {. }
$$




\section{The 6 operations}

4.1. The functor $\mathscr{R h o m}(-,-)$. Let $\mathscr{X}$ be an S-stack locally of finite type. As in any topos, one can define internal hom $\mathscr{R} h o m_{\mathscr{X}_{\text {lis-ét }}}(\mathrm{F}, \mathrm{G})$ for any $\mathrm{F} \in \mathrm{D}^{-}(\mathscr{X})$ and $\mathrm{G} \in \mathrm{D}^{+}(\mathscr{X})$.

Lemma 4.1.1. Let $\mathrm{F} \in \mathrm{D}_{c}^{-}(\mathscr{X})$ and $\mathrm{G} \in \mathrm{D}_{c}^{+}(\mathscr{X})$, and let $j$ be an integer. Then the restriction of the sheaf $\mathscr{H}^{j}\left(\mathscr{R} h m_{\mathscr{X}_{\text {lis-ét }}}(\mathrm{F}, \mathrm{G})\right)$ to the étale topos of any object $\mathrm{U} \in \operatorname{Lisse-\operatorname {Et}}(\mathscr{X})$ is canonically isomorphic to $\mathscr{E} x t_{\mathrm{U}_{\text {ét }}^{j}}^{j}\left(\mathrm{~F}_{\mathrm{U}}, \mathrm{G}_{\mathrm{U}}\right)$, where $\mathrm{F}_{\mathrm{U}}$ and $\mathrm{G}_{\mathrm{U}}$ denote the restrictions to $\mathrm{U}_{\text {ét }}$.

Proof: The sheaf $\mathscr{H}^{j}\left(\mathscr{R} h o m_{\mathscr{X}_{\text {lis-ét }}}(\mathrm{F}, \mathrm{G})\right)$ is the sheaf associated to the presheaf which to any smooth affine $\mathscr{X}$-scheme $\mathrm{U}$ associates $\operatorname{Ext}_{\mathscr{X}_{\text {lis-ét|U }}^{j}}(\mathrm{~F}, \mathrm{G})$, where $\mathscr{X}_{\text {lis-ét|U }}$ denotes the localized topos. Let $\epsilon: \mathscr{X}_{\text {lis-ét|U }} \rightarrow \mathrm{U}_{\text {ét }}$ be the morphism of topos induced by the inclusion of Étale(U) into Lisse- $\operatorname{Et}(\mathscr{X})_{\mid \mathrm{U}}$. Then since $\mathrm{F}$ and $\mathrm{G}$ have constructible cohomology, the natural maps $\epsilon^{*} \epsilon_{*} \mathrm{~F} \rightarrow \mathrm{F}$ and $\epsilon^{*} \epsilon_{*} \mathrm{G} \rightarrow \mathrm{G}$ are isomorphisms in $\mathrm{D}\left(\mathscr{X}_{\text {lis-ét } \mid \mathrm{U}}\right)$. By the projection formula it follows that

$$
\operatorname{Ext}_{\mathscr{X}_{\text {lis-ét|U }}^{j}}^{j}(F, G) \simeq \operatorname{Ext}_{\mathscr{X}_{\text {lis-ét|U }}^{j}}^{j}\left(\epsilon^{*} \epsilon_{*} \mathrm{~F}, \epsilon^{*} \epsilon_{*} \mathrm{G}\right) \simeq \operatorname{Ext}_{\mathrm{U}_{\text {ét }}}^{j}\left(\epsilon_{*} \mathrm{~F}, \epsilon_{*} \mathrm{G}\right)
$$

Sheafifying this isomorphism we obtain the isomorphism in the lemma.

Corollary 4.1.2. If $\mathrm{F} \in \mathrm{D}_{c}^{-}(\mathscr{X})$ and $\mathrm{G} \in \mathrm{D}_{c}^{+}(\mathscr{X})$, the complex $\mathscr{R} h_{\mathscr{X}_{\text {lis-ét }}}(\mathrm{F}, \mathrm{G})$ lies in $\mathrm{D}_{c}^{+}(\mathscr{X})$.

Proof: By the previous lemma and the constructibility of the cohomology sheaves of $\mathrm{F}$ and $\mathrm{G}$, it suffices to prove the following statement: Let $f: \mathrm{V} \rightarrow \mathrm{U}$ be a smooth morphism of schemes of finite type over $\mathrm{S}$, and let $\mathrm{F} \in \mathrm{D}_{c}^{-}\left(\mathrm{U}_{\text {ét }}\right)$ and $\mathrm{G} \in \mathrm{D}_{c}^{+}\left(\mathrm{U}_{\text {ét }}\right)$. Then the natural map $f^{*} \mathscr{R} h o m_{\mathrm{U}_{\text {ét }}}(\mathrm{F}, \mathrm{G}) \rightarrow \mathscr{R} h o m_{\mathrm{V}_{\text {ét }}}\left(f^{*} \mathrm{~F}, f^{*} \mathrm{G}\right)$ is an isomorphism as we saw in the proof of 3.5 .2 (see [5], I.7.2).

Proposition 4.1.3. Let $\mathrm{X} / \mathrm{S}$ be an $\mathrm{S}$-scheme locally of finite type and $\mathrm{X} \rightarrow \mathscr{X}$ be a smooth surjection. Let $\mathrm{X}_{\bullet} \rightarrow \mathscr{X}$ be the resulting strictly simplicial space. Then for $\mathrm{F} \in \mathrm{D}_{c}^{-}\left(\mathscr{X}_{\text {lis-ét }}\right)$ and $\mathrm{G} \in \mathrm{D}_{c}^{+}\left(\mathscr{X}_{\text {lis-ét }}\right)$ there is a canonical isomorphism

$$
\left.\mathscr{R h o m} \mathscr{X}_{\text {lis-ét }}(\mathrm{F}, \mathrm{G})\right|_{\mathrm{X} \bullet \text {,ét }} \simeq \mathscr{R} h o m_{\mathrm{X}}{ }_{\bullet \text { ét }}\left(\left.\mathrm{F}\right|_{\mathrm{x}_{\bullet}, \text { ét }},\left.\mathrm{G}\right|_{\mathrm{X}_{\bullet} \text { ét }}\right) .
$$

In particular, $\mathscr{R} h o m_{\mathrm{X}_{\bullet} \text { ét }}\left(\left.\mathrm{F}\right|_{\mathrm{X}_{\bullet}, \text { ét }},\left.\mathrm{G}\right|_{\mathrm{X}_{\bullet} \text {,ét }}\right)$ maps under the equivalence of categories $\mathrm{D}_{c}\left(\mathrm{X}_{\bullet}\right.$,ét $) \simeq$ $\mathrm{D}_{c}\left(\mathscr{X}_{\text {lis-ét }}\right)$ to $\mathscr{R} h o m_{\mathscr{X}_{\text {lis-ét }}}(\mathrm{F}, \mathrm{G})$. 
Proof: Let $\mathscr{X}_{\text {lis-ét|X. }}$ denote the strictly simplicial localized topos and consider the morphisms of topos

$$
\mathscr{X}_{\text {lis-ét }} \stackrel{\pi}{\longleftarrow} \mathscr{X}_{\text {lis-ét } \mid \mathrm{X}} \stackrel{\epsilon}{\longrightarrow} \mathrm{X}_{\bullet} \text {,ét } .
$$

Let $\mathrm{F}_{\text {ét }}:=\epsilon_{*} \pi^{*} \mathrm{~F}$ and $\mathrm{G}_{\text {ét }}:=\epsilon_{*} \pi^{*} \mathrm{G}$. Since $\mathrm{F}, \mathrm{G} \in \mathrm{D}_{c}\left(\mathscr{X}_{\text {lis-ét }}\right)$, the natural maps $\mathrm{F} \simeq \mathrm{R} \pi_{*} \epsilon^{*} \mathrm{~F}_{\text {ét }}$ and $\mathrm{G} \simeq \mathrm{R} \pi_{*} \epsilon^{*} \mathrm{G}$ are isomorphisms (2.2.3). Using the projection formula we then obtain

$$
\begin{aligned}
& \left.\mathscr{R} h o m_{\mathscr{X}_{\text {lis-ét }}}(\mathrm{F}, \mathrm{G})\right|_{\mathrm{X}} \text {,ét } \simeq \epsilon_{*} \pi^{*} \mathscr{R} h o m_{\mathscr{X}_{\text {lis-ét }}}(\mathrm{F}, \mathrm{G}) \\
& \simeq \epsilon_{*} \pi^{*} \pi_{*} \mathscr{R} h o m_{\mathscr{X}_{\text {lis-ét|x. }}}\left(\epsilon^{*} \mathrm{~F}_{\text {ét }}, \epsilon^{*} \mathrm{G}_{\text {ét }}\right) \\
& \simeq \epsilon_{*} \mathscr{R} h o m_{\mathscr{X}_{\text {lis-ét } \mid \mathrm{X}}}\left(\epsilon^{*} \mathrm{~F}_{\text {ét }}, \epsilon^{*} \mathrm{G}_{\text {ét }}\right) \\
& \simeq \mathscr{R} h o m_{\mathrm{X}_{\bullet}, \text { ét }}\left(\mathrm{F}_{\text {ét }}, \mathrm{G}_{\text {ét }}\right) \text {. }
\end{aligned}
$$

4.2. The functor $f^{*}$. The lisse-étale site is not functorial (cf. [6], 5.3.12): a morphism of stacks does not induce a general a morphism between corresponding lisse-étale topos. In [19], a functor $f^{*}$ is constructed on $\mathrm{D}_{c}^{+}$using cohomological descent. Using the results of 2.2 .3 which imply that we have cohomological descent also for unbounded complexes, the construction of [19] can be used to define $f^{*}$ on the whole category $\mathscr{D}_{c}$.

Let us review the construction here. Let $f: \mathscr{X} \rightarrow \mathscr{Y}$ be a morphism of algebraic S-stacks locally of finite type. Choose a commutative diagram

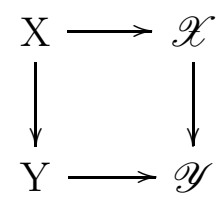

where the horizontal lines are presentations inducing a commutative diagram of strict simplicial spaces

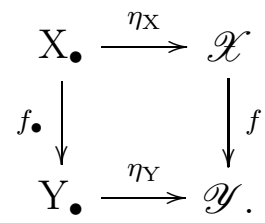

We get a diagram of topos 


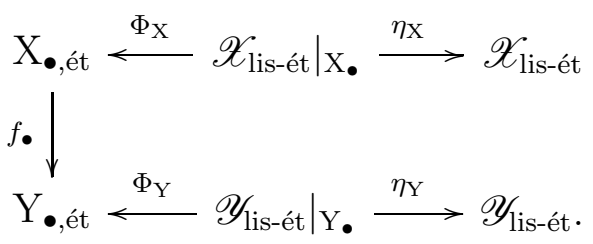

By 2.2.6 the horizontal morphisms induce equivalences of topos

$$
\mathrm{D}_{c}\left(\mathscr{X}_{\text {lis-ét }}\right) \simeq \mathrm{D}_{c}\left(\mathrm{X}_{\bullet} \text {,ét }\right), \quad \mathrm{D}_{c}\left(\mathscr{Y}_{\text {lis-ét }}\right) \simeq \mathrm{D}_{c}\left(\mathrm{Y}_{\bullet} \text {,ét }\right)
$$

We define the functor $f^{*}: \mathrm{D}_{c}\left(\mathscr{Y}_{\text {lis-ét }}\right) \rightarrow \mathrm{D}_{c}\left(\mathscr{X}_{\text {lis-ét }}\right)$ to be the composite

$$
\mathrm{D}_{c}\left(\mathscr{Y}_{\text {lis-ét }}\right) \simeq \mathrm{D}_{c}\left(\mathrm{Y}_{\bullet}, \text { ét }\right) \stackrel{f_{\bullet}^{*}}{\longrightarrow} \mathrm{D}_{c}\left(\mathrm{X}_{\bullet}, \text { ét }\right) \simeq \mathrm{D}_{c}\left(\mathscr{X}_{\text {lis-ét }}\right)
$$

where $f_{\bullet}^{*}$ denotes the derived pullback functor induced by the morphism of topos $f_{\bullet}: \mathrm{X}_{\bullet}$,ét $\rightarrow$ $Y_{\bullet}$ ét. Note that $f^{*}$ takes distinguished triangles to distinguished triangles since this is true for $f_{\bullet}^{*}$.

Proposition 4.2.1. Let $\mathrm{A} \in \mathrm{D}_{c}^{-}(\mathscr{Y})$ and let $\mathrm{B} \in \mathrm{D}_{c}^{+}(\mathscr{X})$. Then there is a canonical isomorphism

$$
f_{*} \mathscr{R h o m}\left(f^{*} \mathrm{~A}, \mathrm{~B}\right) \simeq \mathscr{R h o m}\left(\mathrm{A}, f_{*} \mathrm{~B}\right) .
$$

where we write $f_{*}$ for $\mathrm{R} f_{*}$.

Proof: By 4.1.3 and [19], we have

$$
\left.\mathrm{R} f_{*} \mathscr{R h o m}\left(f^{*} \mathrm{~A}, \mathrm{~B}\right)\right|_{\mathrm{Y}_{\bullet, \mathrm{e} t}} \simeq \mathrm{R} f_{\bullet *} \mathscr{R h o m} \mathrm{X}_{\bullet, \text { ét }}\left(\left.f_{\bullet}^{*} \mathrm{~A}\right|_{\mathrm{Y}_{\bullet}, \text { ét }},\left.\mathrm{B}\right|_{\mathrm{X}_{\bullet, \text { ét }}}\right) \text {. }
$$

The result therefore follows from the usual adjunction

$$
\mathrm{R} f_{\bullet *} \mathscr{R} h o m_{\mathrm{X} \bullet \text { ét }}\left(f_{\bullet}^{*}\left(\left.\mathrm{~A}\right|_{\mathrm{Y}_{\bullet} \text { ét }}\right),\left.\mathrm{B}\right|_{\mathrm{X} \bullet \text { ét }}\right) \simeq \mathscr{R} h o m_{\mathrm{Y}_{\bullet \text { ét }}}\left(\left.\mathrm{A}\right|_{\bullet, \text { ét }},\left.f_{*} \mathrm{~B}\right|_{\mathrm{X}} \text { ét }\right) .
$$

Remark 4.2.2. Its definitely hopeless to generalize 4.2 .1 to $\mathrm{B} \in \mathrm{D}_{c}(\mathscr{X})$ because in general $\mathrm{R} f_{*}$ does not map $\mathrm{D}_{c}$ to itself (for example consider $\mathrm{B} \mathbb{G}_{m} \rightarrow \operatorname{Spec}(k)$ and $\mathrm{B}=\oplus_{i \geq 0} \Lambda[i]$ ).

Remark 4.2.3. One can even show that 4.2.1 still holds for arbitrary $\mathrm{A} \in \mathrm{D}_{c}(\mathscr{Y})$, but the geometric significance is unclear because it is an equality of non constructible complexes. 
4.3. Definition of $\mathrm{R} f_{!}, f^{!}$. Let $f: \mathscr{X} \rightarrow \mathscr{Y}$ be a morphism of stacks (locally of finite type over $\mathrm{S})$ of finite type. Recall ([16], corollaire 18.4 .4$)$ that $\mathrm{R} f_{*}$ maps $\mathrm{D}_{c}^{+}\left(\mathscr{X}_{\text {lis-ét }}\right)$ to $\mathrm{D}_{c}^{+}\left(\mathscr{Y}_{\text {lis-ét }}\right)$.

Definition 4.3.1. We define

$$
\mathrm{R} f_{!}: \mathrm{D}_{c}^{-}\left(\mathscr{X}_{\text {lis-ét }}\right) \rightarrow \mathrm{D}_{c}^{-}\left(\mathscr{Y}_{\text {lis-ét }}\right)
$$

by the formula

$$
\mathrm{R} f_{!}=\mathrm{D}_{\mathscr{Y}} \circ \mathrm{R} f_{*} \circ \mathrm{D}_{\mathscr{X}},
$$

and

$$
f^{!}: \mathrm{D}_{c}^{-}\left(\mathscr{Y}_{\text {lis-ét }}\right) \rightarrow \mathrm{D}_{c}^{-}\left(\mathscr{X}_{\text {lis-ét }}\right)
$$

by the formula

$$
f^{!}=\mathrm{D}_{\mathscr{X}} \circ f^{*} \circ \mathrm{D}_{\mathscr{Y}} .
$$

By construction, one has

$$
f^{!} \Omega_{\mathscr{Y}}=\Omega_{\mathscr{X}} .
$$

Proposition 4.3.2. Let $\mathrm{A} \in \mathrm{D}_{c}^{-}\left(\mathscr{X}_{\text {lis-ét }}\right)$ and $\mathrm{B} \in \mathrm{D}_{c}^{-}\left(\mathscr{Y}_{\text {lis-ét }}\right)$. Then there is a (functorial) adjunction formula

$$
\mathrm{R} f_{*} \mathscr{R h o m}\left(\mathrm{A}, f^{!} \mathrm{B}\right)=\mathscr{R} h o m\left(\mathrm{R} f_{!} \mathrm{A}, \mathrm{B}\right)
$$

Proof: We write $\mathrm{D}$ for $\mathrm{D}_{\mathscr{X}}, \mathrm{D}_{\mathscr{Y}}$ and $\mathrm{A}^{\prime}=\mathrm{D}(\mathrm{A}) \in \mathrm{D}_{c}^{+}(\mathscr{X})$. One has

$$
\begin{aligned}
\mathscr{R h o m}\left(\mathrm{R} f_{!} \mathrm{D}\left(\mathrm{A}^{\prime}\right), \mathrm{B}\right) & =\mathscr{R h o m}\left(\mathrm{D}\left(\mathrm{R} f_{*} \mathrm{~A}^{\prime}\right), \mathrm{B}\right) \\
& =\mathscr{R} h o m\left(\mathrm{D}(\mathrm{B}), \mathrm{R} f_{*} \mathrm{~A}^{\prime}\right) \text { (3.5.9) } \\
& =\mathrm{R} f_{*} \mathscr{R} h o m\left(f^{*} \mathrm{D}(\mathrm{B}), \mathrm{A}^{\prime}\right) \\
& =\mathrm{R} f_{*} \mathscr{R} h o m\left(\mathrm{D}\left(\mathrm{A}^{\prime}\right), f^{!} \mathrm{B}\right)
\end{aligned}
$$

\subsection{Projection formula.}

Lemma 4.4.1. Let $\mathrm{A}, \mathrm{B} \in \mathrm{D}_{c}(\mathscr{X})$.

(i) One has

$$
\mathscr{R h o m}(\mathrm{A}, \mathrm{B})=\mathrm{D}_{\mathscr{X}}\left(\mathrm{A} \stackrel{\mathrm{L}}{\otimes} \mathrm{D}_{\mathscr{X}}(\mathrm{B})\right) .
$$

(ii) If $\mathrm{A}, \mathrm{B} \in \mathrm{D}_{c}^{-}(\mathscr{X})$, then $\mathrm{A} \stackrel{\mathrm{L}}{\otimes} \mathrm{B} \in \mathrm{D}_{c}^{-}(\mathscr{X})$.

(iii) If $\mathrm{A} \in \mathrm{D}_{c}^{-}(\mathscr{X}), \mathrm{B} \in \mathrm{D}_{c}^{+}(\mathscr{X})$, then $\mathscr{R h o m}(\mathrm{A}, \mathrm{B}) \in \mathrm{D}_{c}^{+}(\mathscr{X})$. 
THE SIX OPERATIONS FOR SHEAVES ON ARTIN STACKS I: FINITE COEFFICIENTS

Proof: Let $\Omega_{\mathscr{X}}$ be the dualizing complex of $\mathscr{X}$.

$$
\begin{aligned}
\mathscr{R h o m}(\mathrm{A}, \mathrm{B}) & =\mathscr{R h o m}\left(\mathrm{D}_{\mathscr{X}}(\mathrm{B}), \mathscr{R} h o m\left(\mathrm{~A}, \Omega_{\mathscr{X}}\right)\right) \\
& =\mathscr{R} \operatorname{l3.5.9}) \\
& \left.=\mathrm{D}_{\mathscr{X}}\left(\mathrm{A} \stackrel{\mathrm{L}}{\otimes} \mathrm{D}_{\mathscr{X}}(\mathrm{B}) \stackrel{\mathrm{L}}{\otimes} \mathrm{A}, \Omega_{\mathscr{X}}\right)\right)
\end{aligned}
$$

proving (i). For (ii), using truncations, one can assume that A, B are sheaves : the result is obvious in this case. Statement (iii) follows from the two previous points.

Corollary 4.4.2. Let $f: \mathscr{X} \rightarrow \mathscr{Y}$ be a morphism as in 4.3 , and let $\mathrm{B} \in \mathrm{D}_{c}^{-}(\mathscr{Y}), \mathrm{A} \in \mathrm{D}_{c}^{-}(\mathscr{X})$. One has the projection formula

$$
\mathrm{R} f_{!}\left(\mathrm{A} \stackrel{\mathrm{L}}{\otimes} f^{*} \mathrm{~B}\right)=\mathrm{R} f_{!} \mathrm{A} \stackrel{\mathrm{L}}{\otimes} \mathrm{B} .
$$

Proof: Notice that the left-hand side is well defined by 4.4.1. One has

$$
\begin{aligned}
\mathrm{R} f_{!}\left(\mathrm{A} \otimes f^{*} \mathrm{~B}\right) & =\mathrm{D}_{\mathscr{Y}} \circ \mathrm{R} f_{*} \circ \mathrm{D} \mathscr{X}\left(\mathrm{A} \stackrel{\mathrm{L}}{\mathrm{D}} \mathscr{X}^{\prime} f_{\mathscr{Y}} \mathrm{B}\right) \\
& =\mathrm{D}_{\mathscr{Y}} \circ \mathrm{R} f_{*}\left(\mathscr{R} h o m\left(\mathrm{~A}, f^{!} \mathrm{D}_{\mathscr{Y}} \mathrm{B}\right)\right) \text { (4.4.1) } \\
& =\mathrm{D}_{\mathscr{Y}}\left(\mathscr{R} h o m\left(\mathrm{R} f_{!} \mathrm{A}, \mathrm{D}_{\mathscr{Y}} \mathrm{B}\right)\right)(4.3 .2) \\
& =\mathrm{R} f_{!} \mathrm{A} \stackrel{\mathrm{L}}{\otimes} \mathrm{B}(\text { (4.4.1) and (3.5.7). }
\end{aligned}
$$

Corollary 4.4.3. For all $\mathrm{A} \in \mathrm{D}_{c}^{+}(\mathscr{Y}), \mathrm{B} \in \mathrm{D}_{c}^{-}(\mathscr{Y})$, one has $f^{!} \mathscr{R} h o m(\mathrm{~A}, \mathrm{~B})=\mathscr{R} h o m\left(f^{*} \mathrm{~A}, f^{!} \mathrm{B}\right)$.

Proof: By lemma 4.4.1 and biduality, the formula reduces to the formula

$$
f^{*}(\mathrm{~A} \stackrel{\mathbf{L}}{\otimes} \mathrm{D}(\mathrm{B}))=f^{*} \mathrm{~A} \stackrel{\mathbf{L}}{\otimes} f^{*} \mathrm{D}(\mathrm{B}) .
$$

Using suitable presentation, one is reduced to the obvious formula

$$
f_{\bullet}^{*}\left(\mathrm{~A}_{\bullet} \stackrel{\mathrm{L}}{\otimes} \mathrm{B}_{\bullet}\right)=f_{\bullet}^{*} \mathrm{~A}_{\bullet} \stackrel{\mathrm{L}}{\otimes} f_{\bullet}^{*} \mathrm{~B}_{\bullet}
$$

for a morphism $f_{\bullet}$ of stricltly simplicial étale topos.

4.5. Computation of $f^{!}$for $f$ smooth. Let $f: \mathscr{X} \rightarrow \mathscr{Y}$ be a smooth morphism of stacks of relative dimension $d$. Using 2.3.4, one gets immediately the formula

$$
f^{*} \Omega_{\mathscr{Y}}=\Omega_{\mathscr{X}}\langle-d\rangle
$$

(choose a presentation of $\mathrm{Y} \rightarrow \mathscr{Y}$ and then a presentation $\mathrm{X} \rightarrow \mathscr{X}_{\mathrm{Y}}$; the morphism $\mathrm{X} \rightarrow \mathrm{Y}$ being smooth, one checks that these two complexes coincide on $\mathscr{X}_{\text {lis-ét|X }}$ and have zero negative $\mathscr{E} x t$ 's). 
Lemma 4.5.1. Let $\mathrm{A} \in \mathrm{D}_{c}(\mathscr{Y})$. Then, the canonical morphism

$$
f^{*} \mathscr{R h o m}\left(\mathrm{A}, \Omega_{\mathscr{Y}}\right) \rightarrow \mathscr{R h o m}\left(f^{*} \mathrm{~A}, f^{*} \Omega_{\mathscr{Y}}\right)
$$

is an isomorphism.

Proof: Using 3.4.1, one is reduced to the usual statement for étale sheaves on algebraic spaces. Because, in this case, both $\Omega_{\mathscr{Y}}$ and $f^{*} \Omega_{\mathscr{Y}}$ are of finite injective dimension, one can assume that $\mathrm{A}$ is bounded or even a sheaf. The assertion is well-known in this case (by dévissage, one reduces to $\mathrm{A}=\Lambda_{\mathscr{Y}}$ in which case the assertion is trivial, cf. [5], exp. I).

Corollary 4.5.2. Let $f: \mathscr{X} \rightarrow \mathscr{Y}$ be a smooth morphism of stacks of relative dimension $d$. One has $f^{!}=f^{*}\langle d\rangle$.

Let $j: \mathscr{U} \rightarrow \mathscr{X}$ be an open immersion. Let us denote for a while $j_{i}$ the extension by zero functor : it is an exact functor on the category sheaves preserving constructibility and therefore passes to the derive category $\mathrm{D}_{c}$.

Proposition 4.5.3. One has $j^{!}=j^{*}$ and $j_{!}=j_{i}$.

Proof: The first equality is a particular case of 4.5.2. Because $j^{*}$ has a left adjoint $j_{i}$ which is exact, it preserves (homotopical) injectivity. Let A, B be constructible complexes on $\mathscr{U}, \mathscr{X}$ respectively and assume that $\mathrm{B}$ is homotopically injective. One has

$$
\begin{aligned}
\operatorname{Rhom}\left(j_{i} \mathrm{~A}, \mathrm{~B}\right) & =\operatorname{Hom}\left(j_{i} \mathrm{~A}, \mathrm{~B}\right) \\
& =\operatorname{Hom}\left(\mathrm{A}, j^{*} \mathrm{~B}\right) \text { (adjunction) } \\
& =\operatorname{Rhom}\left(\mathrm{A}, j^{*} \mathrm{~B}\right)
\end{aligned}
$$

Taking $\mathscr{H}^{0}$, one obtains that $j^{*}$ is the right adjoint of $j_{i}$ proving the lemma because $j^{!}=j^{*}$ is the right adjoint of $j !$

4.6. Computation of $\mathrm{R} i_{1}$ for $i$ a closed immersion. Let $i: \mathscr{X} \hookrightarrow \mathscr{Y}$ be a closed immersion and $\mathscr{U}=\mathscr{Y}-\mathscr{X} \hookrightarrow \mathscr{Y}$ the open immersion of the complement : both are representable. We define the cohomology with support on $\mathscr{X}$ for any $\mathrm{F} \in \mathscr{X}_{\text {lis-ét }}$ as follows. First, for any $\mathrm{Y} \rightarrow \mathscr{Y}$ in Lisse-Et($(\mathscr{Y})$, the pull-back $\mathrm{Y}_{\mathscr{U}} \rightarrow \mathscr{U}$ is in Lisse-Et($(\mathscr{U})$ and $\mathrm{Y}_{\mathscr{U}} \rightarrow \mathscr{U} \rightarrow \mathscr{Y}$ is in $\operatorname{Lisse-Et}(\mathscr{Y})$. Then, we define $\underline{\mathrm{H}}_{\mathscr{X}}^{0}(\mathrm{~F})$

$$
\Gamma\left(\mathrm{Y}, \underline{\mathrm{H}}_{\mathscr{X}}^{0}(\mathrm{~F})\right)=\operatorname{ker}\left(\Gamma(\mathrm{Y}, \mathrm{F}) \rightarrow \Gamma\left(\mathrm{Y}_{\mathscr{U}}, \mathrm{F}\right)\right)
$$

and $\mathrm{R} \Gamma_{\mathscr{X}}$ is the total derived functor of the left exact functor $\mathscr{H}_{\mathscr{X}}^{0}$. 
Lemma 4.6.1. One has $\Omega_{\mathscr{X}}=i^{*} \mathrm{R} \Gamma_{\mathscr{X}}\left(\Omega_{\mathscr{Y}}\right)$.

Proof: If $i$ is a closed immersion of schemes (or algebraic spaces), one has a canonical (and functorial) isomorphism, simply because $i^{*} \underline{H}_{\mathscr{X}}^{0}$ is the right adjoint of $i_{*}$. If $\mathrm{K}$ denotes one of the objects on the two sides of the equality to be proven, one has therefore $\mathscr{E} x t^{i}(\mathrm{~K}, \mathrm{~K})=0$ for $i<0$. Therefore, these isomorphisms glue (use theorem 3.2.2 of [7] as before).

Proposition 4.6.2. The functor $\mathrm{B} \mapsto i^{*} \mathrm{R} \Gamma_{\mathscr{X}}(\mathrm{B})$ is the right adjoint of $i_{*}$, and therefore coincides with $i^{!}$. More generally, one has

$$
\mathscr{R h o m}\left(i_{*} \mathrm{~A}, \mathrm{~B}\right)=i_{*} \mathscr{R h o m}\left(\mathrm{A}, i^{*} \underline{\mathrm{H}}_{\mathscr{X}}^{0}(\mathrm{~B})\right)
$$

for all $\mathrm{A} \in \mathrm{D}(\mathscr{X}), \mathrm{B} \in \mathrm{D}(\mathscr{Y})$. Moreover, one has one has $i_{!}=i_{*}$ and has a right adjoint, the sections with support on $\mathscr{X}$.

Proof: If A, B are sheaves, one has the usual adjunction formula

$$
\operatorname{hom}\left(i_{*} \mathrm{~A}, \mathrm{~B}\right)=i_{*} \operatorname{hom}\left(\mathrm{A}, i^{*} \underline{\mathrm{H}}_{\mathscr{X}}^{0}(\mathrm{~B})\right) .
$$

Because $i_{*}$ is exact, it's right adjoint sends homotopically injective complexes to homotopically injective complexes. The derived version follows. One gets therefore

$$
\begin{aligned}
i_{!} \mathrm{A} & =\mathscr{R h o m}\left(i_{*} \mathscr{R h o m}\left(\mathrm{A}, \Omega_{\mathscr{X}}\right), \Omega_{\mathscr{Y}}\right) \\
& =i_{*} \mathscr{R h o m}\left(\mathscr{R h o m}\left(\mathrm{A}, \Omega_{\mathscr{X}}\right), i^{*} \mathrm{RH}_{\mathscr{X}}^{0}\left(\Omega_{\mathscr{Y}}\right)\right) \\
& =i_{*} \mathscr{R h o m}\left(\mathscr{R h o m}\left(\mathrm{A}, \Omega_{\mathscr{X}}\right), \Omega_{\mathrm{X}}\right) \\
& =i_{*} \mathrm{~A}
\end{aligned}
$$

4.7. Computation of $f^{!}$for a universal homeomorphism. By universal homeomorphism we mean a representable, radiciel and surjective morphism. By Zariski's main theorem, such a morphism is finite.

In the schematic situation, we know that such a morphism induces an isomorphism of the étale topos ([3], VIII.1.1). In particular, $f^{*}$ is also a right adjoint of $f_{*}$. Being exact, one gets in this case an identification $f^{*}=f^{!}$. In particular, $f^{*}$ identifies the corresponding dualizing complexes. Exactly as in the proof of 4.6.1, one gets

Lemma 4.7.1. Let $f: \mathscr{X} \rightarrow \mathscr{Y}$ be a universal homeomorphism of stacks. One has $f^{*} \Omega_{\mathscr{X}}=\Omega_{\mathscr{Y}}$.

One gets therefore 
Corollary 4.7.2. Let $f: \mathscr{X} \rightarrow \mathscr{Y}$ be a universal homeomorphism of stacks. One has one has $f^{!}=f^{*}$ and $\mathrm{R} f_{!}=\mathrm{R} f_{*}$.

Proof: One has

$$
\begin{aligned}
f^{!} \mathrm{A} & =\mathscr{R h o m}\left(f^{*} \mathscr{R h o m}\left(\mathrm{A}, \Omega_{\mathscr{Y}}\right), \Omega_{\mathscr{X}}\right) \\
& =\mathscr{R h o m}\left(\mathscr{R h o m}\left(f^{*} \mathrm{~A}, f^{*} \Omega_{\mathscr{Y}}\right), \Omega_{\mathscr{X}}\right) \\
& =\mathscr{R h o m}\left(\mathscr{R h o m}\left(f^{*} \mathrm{~A}, \Omega_{\mathscr{X}}\right), \Omega_{\mathscr{X}}\right) \\
& =f^{*} \mathrm{~A}
\end{aligned}
$$

The last formula follows by adjunction.

4.8. Computation of $\mathrm{R} f$ ! via hypercovers. Let $\mathrm{Y}$ be an $\mathrm{S}$-scheme of finite type and $f$ : $\mathscr{X} \rightarrow \mathrm{Y}$ a morphism of finite type from an algebraic stack $\mathscr{X}$. Let $\mathrm{X} \bullet \rightarrow \mathscr{X}$ be a smooth hypercover by algebraic spaces, and for each $n$ let $d_{n}$ denote the locally constant function on $\mathrm{X}_{n}$ which is the relative dimension over $\mathscr{X}$. By the construction, the restriction of the dualizing complex $\Omega_{\mathscr{X}}$ to each $\mathrm{X}_{n \text {,ét }}$ is canonically isomorphic to the dualizing complex $\mathrm{K}_{\mathrm{X}_{n}}=\Omega_{\mathscr{X}_{n}}\left\langle-d_{n}\right\rangle$ of $\mathrm{X}_{n}$. Let $\mathrm{K}_{\mathrm{X}}$. denote the restriction of $\Omega_{\mathscr{X}}$ to $\mathrm{X}_{\bullet}$,ét.

Let $\mathrm{L} \in \mathrm{D}_{c}^{-}(\mathscr{X})$, and let $\left.\mathrm{L}\right|_{\mathrm{X}}$. denote the restriction of $\mathrm{L}$ to $\mathrm{X}_{\bullet}$,ét. Then $\left.\mathrm{D}_{\mathscr{X}}(\mathrm{L})\right|_{\mathrm{x}}$. is isomorphic to $\mathrm{D}_{\mathrm{X}_{\bullet}}\left(\left.\mathrm{L}\right|_{\mathrm{X}}\right):=\mathscr{R} \operatorname{hom}_{\mathrm{X}_{\bullet} \text { ét }}\left(\left.\mathrm{L}\right|_{\mathrm{X}_{\bullet}}, \mathrm{K}_{\mathrm{X}} \cdot\right)$. In particular, the restriction of $\mathrm{R} f_{!} \mathrm{L}$ to $\mathrm{Y}_{\text {ét }}$ is canonically isomorphic to

$$
\mathscr{R h o m} \mathrm{Y}_{\text {ét }}\left(\mathrm{R} f_{\bullet *} \mathrm{D}_{\mathrm{X}} \cdot\left(\left.\mathrm{L}\right|_{\mathrm{X}},\right), \mathrm{K}_{\mathrm{Y}}\right) \in \mathrm{D}_{c}\left(\mathrm{Y}_{\text {ét }}\right),
$$

where $f_{\bullet}: \mathrm{X}_{\text {ét }} \rightarrow \mathrm{Y}_{\text {ét }}$ denotes the morphism of topos induced by $f$.

Let $\mathrm{Y}_{\bullet}$,ét denote the simplicial topos obtained by viewing $\mathrm{Y}$ as a constant simplicial scheme. Let $\epsilon: \mathrm{Y}_{\bullet}$,ét $\rightarrow \mathrm{Y}_{\text {ét }}$ denote the canonical morphism of topos, and let $\tilde{f}: \mathrm{X}_{\bullet}$,ét $\rightarrow \mathrm{Y}_{\bullet}$,ét be the morphism of topos induced by $f$. We have $f_{\bullet}=\epsilon \circ \tilde{f}$. As in [19], 2.7, it follows that there is a canonical spectral sequence

$$
\mathrm{E}_{1}^{p q}=\mathrm{R}^{q} f_{p *} \mathrm{D}_{\mathrm{X}_{p}}\left(\left.\mathrm{~L}\right|_{\mathrm{X}_{p}}\right) \Longrightarrow \mathrm{R}^{p+q} f_{\bullet *} \mathrm{D}_{\mathrm{X}}\left(\mathrm{L}_{\mathrm{X}} \cdot\right)
$$

On the other hand, we have

$$
\mathrm{R}^{q} f_{p *} \mathrm{D}_{\mathrm{X}_{p}}\left(\left.\mathrm{~L}\right|_{\mathrm{X}_{p}}\right)=\mathrm{R}^{q} f_{p *} \mathscr{R} h o m\left(\left.\mathrm{~L}\right|_{\mathrm{X}_{p}}, \Omega_{\mathrm{X}_{p}}\left\langle-d_{p}\right\rangle\right) \simeq \mathscr{H}^{q}\left(\mathrm{D}_{\mathrm{Y}}\left(\mathrm{R} f_{p !}\left(\left.\mathrm{L}\right|_{\mathrm{X}_{p}}\left\langle d_{p}\right\rangle\right)\right)\right.
$$

where the second isomorphism is by biduality 3.5.7. Combining all this we obtain

Proposition 4.8.1. There is a canonical spectral sequence

$$
\mathrm{E}_{1}^{p q}=\mathscr{H}^{q}\left(\mathrm{D}_{\mathrm{Y}_{\mathrm{et}}}\left(\left.\mathrm{R} f_{p !} \mathrm{L}\right|_{\mathrm{X}_{p}}\left\langle d_{p}\right\rangle\right)\right) \Longrightarrow \mathscr{H}^{p+q}\left(\mathrm{D}_{\mathrm{Y}_{\mathrm{et}}}\left(\left.\mathrm{R} f_{!} \mathrm{L}\right|_{\mathrm{Y}_{\mathrm{et}}}\right)\right) .
$$


Example 4.8.2. Let $k$ be an algebraically closed field and $\mathrm{G}$ a finite group. We can then compute $\mathrm{H}_{c}^{*}(\mathrm{BG}, \Lambda)$ as follows. We first compute $\mathscr{R} h o m\left(\mathrm{R} \Gamma_{!}(\mathrm{BG}, \Lambda), \Lambda\right)$. Let $\operatorname{Spec}(k) \rightarrow \mathrm{BG}$ be the surjection corresponding to the trivial $\mathrm{G}$-torsor, and let $\mathrm{X} . \rightarrow \mathrm{BG}$ be the 0 -coskeleton. Note that each $\mathrm{X}_{n}$ isomorphic to $\mathrm{G}^{n}$ and in particular is a discrete collection of points. Therefore $\mathrm{R} f_{! p} \Lambda \simeq \operatorname{Hom}\left(\mathrm{G}^{n}, \Lambda\right)$. From this it follows that $\mathscr{R} h o m\left(\mathrm{R} \Gamma_{!}(\mathrm{BG}, \Lambda), \Lambda\right)$ is represented by the standard cochain complex computing the group cohomology of $\Lambda$, and hence $R \Gamma_{!}(\mathrm{BG}, \Lambda)$ is the dual of this complex. In particular, this can be nonzero in infinitely many negative degrees. For example if $G=\mathbb{Z} / \ell$ for some prime $\ell$ and $\Lambda=\mathbb{Z} / \ell$ since in this case the group cohomology $\mathrm{H}^{i}(\mathrm{G}, \mathbb{Z} / \ell) \simeq \mathbb{Z} / \ell$ for all $i \geq 0$.

Example 4.8.3. Let $k$ be an algebraically closed field and $\mathrm{P}$ the affine line $\mathbb{A}^{1}$ with the origin doubled. By definition $\mathrm{P}$ is equal to two copies of $\mathbb{A}^{1}$ glued along $\mathbb{G}_{m}$ via the standard inclusions $\mathbb{G}_{m} \subset \mathbb{A}^{1}$. We can then compute $\mathrm{R} \Gamma_{!}(\mathrm{P}, \Lambda)$ as follows. Let $j_{i}: \mathbb{A}^{1} \hookrightarrow \mathrm{P}(i=1,2)$ be the two open immersions, and let $h: \mathbb{G}_{m} \hookrightarrow \mathrm{P}$ be the inclusion of the overlaps. We then have an exact sequence

$$
0 \rightarrow h_{!} \Lambda \rightarrow j_{1 !} \Lambda \oplus j_{2 !} \Lambda \rightarrow \Lambda \rightarrow 0 .
$$

From this we obtain a long exact sequence

$$
\cdots \rightarrow \mathrm{H}_{c}^{i}\left(\mathbb{G}_{m}, \Lambda\right) \rightarrow \mathrm{H}_{c}^{i}\left(\mathbb{A}^{1}, \Lambda\right) \oplus \mathrm{H}_{c}^{i}\left(\mathbb{A}^{1}, \Lambda\right) \rightarrow \mathrm{H}_{c}^{i}(\mathrm{P}, \Lambda) \rightarrow \cdots .
$$

From this sequence one deduces that $\mathrm{H}_{c}^{0}(\mathrm{P}, \Lambda) \simeq \Lambda, \mathrm{H}_{c}^{2}(\mathrm{P}, \Lambda) \simeq \Lambda(1)$, and all other cohomology groups vanish. In particular, the cohomology of $\mathrm{P}$ is isomorphic to the cohomology of $\mathbb{P}^{1}$.

4.9. Purity and the fundamental distinguished triangle. We consider the usual situation of a closed immersion $i: \mathscr{X} \rightarrow \mathscr{Y}$ of stacks, the open immersion of the complement of $\mathscr{Y}$ being $j: \mathscr{U}=\mathscr{Y}-\mathscr{X} \rightarrow \mathscr{Y}$. For any (complex) of sheaves A on $\mathscr{Y}$, one has the exact sequence

$$
0 \rightarrow j_{!} j^{*} \mathrm{~A} \rightarrow \mathrm{A} \rightarrow i_{*} i^{*} \mathrm{~A} \rightarrow 0 .
$$

Therefore, for any $\mathrm{A} \in \mathrm{D}_{c}(\mathscr{Y})$, one has the distinguished triangle (4.5.3)

$$
j ! j^{*} \mathrm{~A} \rightarrow \mathrm{A} \rightarrow i_{*} i^{*} \mathrm{~A}
$$

which by duality gives the distinguished triangle

$$
i_{*} i^{!} \mathrm{A} \rightarrow \mathrm{A} \rightarrow j_{*} j^{*} \mathrm{~A} .
$$

Recall (4.6.2) the formula $i^{!}=\mathrm{R}_{\mathscr{H}}^{0}$. The usual purity theorem for S-schemes gives 
Proposition 4.9.1 (Purity). Assume moreover that $i$ is a closed immersion of smooth S-stacks of codimension c (a locally constant function on $\mathscr{Y}$ ). Then, one has $i^{!} \mathrm{A}=i^{*} \mathrm{~A}(-c)[-2 c]$.

Proof: Let $d$ be the relative dimension of $\mathscr{Y} \rightarrow \mathrm{S}$ and $s$ the dimension of $\mathrm{S}$. The relative dimension of $\mathscr{X}$ is (the restriction to $\mathscr{X}$ of) $d-c$. By 4.5.2, one has

$$
\Omega_{\mathscr{Y}}=\Lambda(d+s)[2 d+2 s] \text { and } \Omega_{\mathscr{X}}=\Lambda(d-c+s)[2 d-2 c-2 e] .
$$

The identity $i ! \Omega_{\mathscr{Y}}=\Omega_{\mathscr{X}}$ gives therefore the formula

$$
i^{!} \Lambda=\Lambda(-c)[-2 c]
$$

By 4.3.2, one has

$$
i_{*} \mathscr{R h o m}\left(i^{!} \Lambda, i^{!} \mathrm{A}\right)=\mathscr{R h o m}\left(i_{!} i^{!} \Lambda, \mathrm{A}\right)
$$

which by adjunction for $i_{*}$ gives a map

$$
i^{*} \mathscr{R h o m}\left(i_{i} i^{!} \Lambda, \mathrm{A}\right) \rightarrow \mathscr{R h o m}\left(i^{!} \Lambda, i^{!} \mathrm{A}\right) .
$$

But the adjunction map (for $i_{!}$) $i_{!} i^{!} \Lambda \rightarrow \Lambda$ dualizes to

$$
\mathscr{R h o m}(\Lambda, \mathrm{A}) \rightarrow \mathscr{R h o m}\left(i_{!} i ! \Lambda, \mathrm{A}\right)
$$

which gives by composition a morphism

$$
i^{*} \mathrm{~A}=i^{*} \mathscr{R h o m}(\Lambda, \mathrm{A}) \rightarrow \mathscr{R h o m}\left(i^{!} \Lambda, i^{!} \mathrm{A}\right)=i^{!} \mathrm{A}(c)[2 c]
$$

which is the usual morphism for closed immersion of schemes. This morphism is compatible with the duality in an obvious sense. The usual purity theorem gives then the proposition, at least for $\mathrm{A} \in \mathrm{D}_{c}^{+}(\mathscr{Y})$. By duality, one gets the proposition for $\mathrm{A} \in \mathrm{D}_{c}^{-}(\mathscr{Y})$, and therefore for $\mathrm{A} \in \mathrm{D}_{c}(\mathscr{Y})$ using the distinguished triangle $\tau_{>0} \mathrm{~A} \rightarrow \mathrm{A} \rightarrow \tau_{\leq 0} \mathrm{~A}$.

\section{BASE CHANGE}

We start with a cartesian diagram of stacks

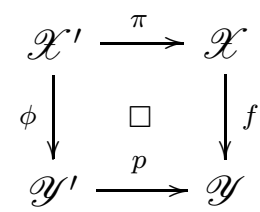

and we would like to prove a natural base change isomorphism

$$
p^{*} \mathrm{R} f_{!}=\mathrm{R} \phi ! \pi^{*}
$$


of functors $\mathrm{D}_{c}(\mathscr{X}) \rightarrow \mathrm{D}_{c}\left(\mathscr{Y}^{\prime}\right)$. Though technically not needed, before proving the general base change Theorem we consider first some simpler cases where one can prove a dual version:

$$
p^{!} \mathrm{R} f_{*}=\mathrm{R} \phi_{*} \pi^{!}
$$

5.1. Smooth base change. In this subsection we prove the base change isomorphism in the case when $p$ (and hence also $\pi$ ) is smooth.

Proof: Because the relative dimension of $p$ and $\pi$ are the same, by 4.5.2, one reduces the formula 5.0 .1 .3 to

$$
p^{*} \mathrm{R} f_{*}=\mathrm{R} \phi_{*} \pi^{*}
$$

By adjunction, one has a morphism $p^{*} \mathrm{R} f_{*} \rightarrow \mathrm{R} \phi_{*} \pi^{*}$ which we claim is an isomorphism (for complexes bounded below this follows immediately from the smooth base change theorem). To prove that this map is an isomorphism, we consider first the case when $\mathscr{Y}^{\prime}$ is algebraic space and show that our morphism restricts to an isomorphism on $\mathscr{Y}_{\text {ét }}^{\prime}$. Since $p$ is representable $\mathscr{X}^{\prime}$

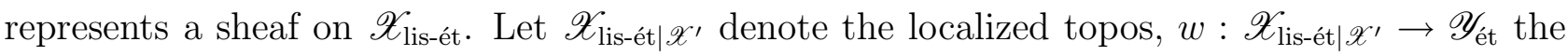
projection, and let $\mathrm{A} \in \mathrm{D}_{c}(\mathscr{X})$ be a complex. Let $\mathrm{X} \rightarrow \mathscr{X}$ be a smooth surjection with $\mathrm{X}$ a scheme, and let $\mathrm{X} \bullet \rightarrow \mathscr{X}$ denote the associated simplicial space. Let $\mathrm{X}_{\bullet}^{\prime}$ denote the base change of $\mathrm{X}_{\bullet}$ to $\mathscr{Y}^{\prime}$. Then $\mathrm{X}_{\bullet}^{\prime}$ defines a hypercover of the initial object in the topos $\mathscr{X}_{\text {lis-ét } \mid \mathscr{X}^{\prime}}$ and hence we have an equivalence of topos $\mathscr{X}_{\text {lis-ét, } \mathscr{X}^{\prime}} \simeq \mathscr{X}_{\text {lis-ét,X'. }}$. Let $w_{\bullet}: \mathscr{X}_{\text {lis-ét|X } \mathbf{\bullet}^{\prime}} \rightarrow \mathscr{Y}_{\text {ét }}^{\prime}$ be the projection. Since the restriction functor from $\mathscr{X}_{\text {lis-ét }}$ to $\mathscr{X}_{\text {lis-ét|x' takes homotopically }}$ injective complexes to homotopically injective complexes (since it has an exact left adjoint),

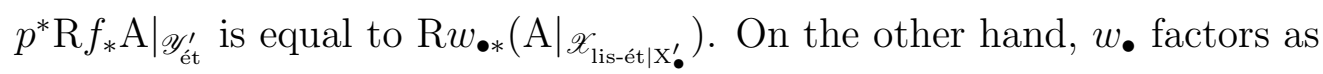

$$
\mathscr{X}_{\text {lis-ét|X'. }} \stackrel{\alpha}{\longrightarrow} \mathrm{X}_{\bullet, \text { ét }}^{\prime} \stackrel{\phi \bullet}{\longrightarrow} \mathscr{Y}_{\text {ét }}^{\prime},
$$

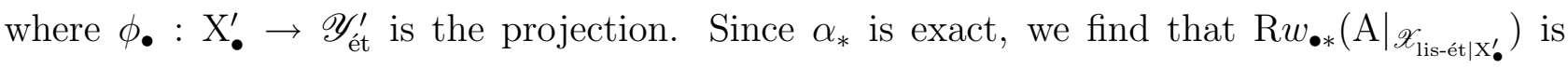
isomorphic to $\mathrm{R} \phi_{\bullet *}\left(\left.\mathrm{~A}\right|_{\mathrm{X}_{\bullet}^{\prime} \text { ét }}\right)$. Similarly, factoring the morphism of topos $\mathscr{X}_{\text {lis-ét }}^{\prime} \simeq \mathscr{X}_{\text {lis-ét } \mid \mathrm{X} \bullet_{\bullet}^{\prime}}^{\prime} \rightarrow \mathscr{Y}_{\text {ét }}^{\prime}$ as

$$
\mathscr{X}_{\text {lis-ét } \mid \mathrm{X}_{\bullet}^{\prime}} \longrightarrow \mathrm{X}_{\bullet, \text { ét }}^{\prime} \stackrel{\phi_{\bullet}}{\longrightarrow} \mathscr{Y}_{\text {êt }}^{\prime}
$$

we see that $\mathrm{R} \phi_{*}\left(\left.\mathrm{~A}\right|_{\mathscr{X}_{\text {lis-ét }}^{\prime}}\right)$ is isomorphic to $\mathrm{R} \phi_{\bullet *}\left(\left.\mathrm{~A}\right|_{\mathrm{X}_{\bullet}^{\prime} \text { ét }}\right)$. We leave to the reader that the resulting isomorphism $\left.\left.p^{*} \mathrm{R} f_{*}(\mathrm{~A})\right|_{\mathscr{Y} \text { ét }} ^{\prime} \rightarrow \mathrm{R} \phi_{*} \pi^{*} \mathrm{~A}\right|_{\mathscr{Y} \text { ét }^{\prime}}$ agrees with the morphism defined above. Thus this proves the case when $p$ is representable. 
For the general case, let $\mathrm{Y}^{\prime} \rightarrow \mathscr{Y}^{\prime}$ denote a smooth surjection with $\mathrm{Y}^{\prime}$ a scheme, so we have a commutative diagram

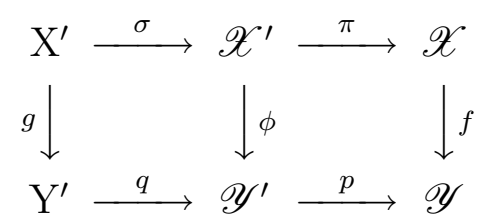

with cartesian squares. Let $\mathrm{A} \in \mathrm{D}_{c}(\mathscr{X})$. To prove that the morphism $p^{*} \mathrm{R} f_{*} \mathrm{~A} \rightarrow \mathrm{R} \phi_{*} \pi^{*} \mathrm{~A}$ is an isomorphism, it suffices to show that the induced morphism $q^{*} p^{*} \mathrm{R} f_{*} \mathrm{~A} \rightarrow q^{*} \mathrm{R} \phi_{*} \pi^{*} \mathrm{~A}$ is an isomorphism. By the representable case, $q^{*} \mathrm{R} \phi_{*} \pi^{*} \mathrm{~A} \simeq \mathrm{R} g_{*} \sigma^{*} \pi^{*} \mathrm{~A}$ so it suffices to prove that the composite

$$
q^{*} p^{*} \mathrm{R} f_{*} \mathrm{~A} \rightarrow \mathrm{R} g_{*} \sigma^{*} \pi^{*} \mathrm{~A}
$$

is an isomorphism. By the construction, this map is equal to the base change morphism for the diagram

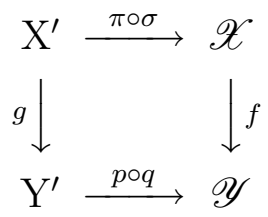

and hence it is an isomorphism by the representable case.

\subsection{Computation of $\mathrm{R} f_{*}$ for proper representable morphisms.}

Proposition 5.2.1. Let $f: \mathscr{X} \rightarrow \mathscr{Y}$ be a proper representable morphism of $\mathrm{S}-$ stacks. Then the functor $\mathrm{R} f_{!}: \mathrm{D}_{c}^{-}(\mathscr{X}) \rightarrow \mathrm{D}_{c}^{-}(\mathscr{Y})$ is canonically isomorphic to $\mathrm{R} f_{*}: \mathrm{D}_{c}^{-}(\mathscr{X}) \rightarrow \mathrm{D}_{c}^{-}(\mathscr{Y})$.

Proof: The key point is the following lemma.

Lemma 5.2.2. There is a canonical morphism $\mathrm{R} f_{*} \Omega_{\mathscr{X}} \rightarrow \Omega_{\mathscr{Y}}$.

Proof: Using 2.3.4 and smooth base change, it suffices to construct a functorial morphism in the case of schemes, and to show that $\mathscr{E} x t^{i}\left(\mathrm{R} f_{*} \Omega_{\mathscr{X}}, \Omega_{\mathscr{Y}}\right)=0$ for $i<0$. Now if $\mathscr{X}$ and $\mathscr{Y}$ are schemes, we have $\Omega_{\mathscr{X}}=f^{!} \Omega_{\mathscr{Y}}$ so we obtain by adjunction and the fact that $\mathrm{R} f_{!}=\mathrm{R} f_{*}$ a morphism $\mathrm{R} f_{*} \Omega_{\mathscr{X}} \rightarrow \Omega_{\mathscr{Y}}$. For the computation of $\mathscr{E} x t$ 's note that

$$
\mathscr{R h o m}\left(\mathrm{R} f_{*} \Omega_{\mathscr{X}}, \Omega_{\mathscr{Y}}\right)=\mathscr{R h o m}\left(\Omega_{\mathscr{X}}, f^{!} \Omega_{\mathscr{Y}}\right)=\Lambda
$$


We define a map $\mathrm{R} f_{*} \circ \mathrm{D}_{\mathscr{X}} \rightarrow \mathrm{D}_{\mathscr{Y}} \circ f_{*}$ by taking the composite

$$
\mathrm{R} f_{*} \mathscr{R h o m}\left(-, \Omega_{\mathscr{X}}\right) \rightarrow \mathscr{R h o m}\left(\mathrm{R} f_{*}(-), \mathrm{R} f_{*} \Omega_{\mathscr{X}}\right) \rightarrow \mathscr{R h o m}\left(\mathrm{R} f_{*}(-), \Omega_{\mathscr{Y}}\right)
$$

To verify that this map is an isomorphism we may work locally on $\mathscr{Y}$. This reduces the proof to the case when $\mathscr{X}$ and $\mathscr{Y}$ are algebraic spaces in which case the result is standard.

5.3. Base change by an immersion. In this subsection we consider the case when $p$ is an immersion.

By replacing $\mathscr{Y}$ by a suitable open substack, one is reduced to the case when $p$ is a closed immersion. Then, 5.0.1.2 follows from the projection formula 4.4.2 as in [10], p.81. Let us recall the argument. Let $\mathrm{A} \in \mathrm{D}_{c}(\mathscr{X})$. Because $p$ is a closed immersion, one has $p^{*} p_{*}=\mathrm{Id}$. One has (projection formula 4.4 .2 for $p$ )

$$
p_{*} p^{*} \mathrm{R} f_{!} \mathrm{A}=p_{*} \Lambda \stackrel{\mathrm{L}}{\otimes} \mathrm{R} f_{!} \mathrm{A}
$$

One has then

$$
\mathrm{R} f_{!} \mathrm{A} \stackrel{\mathrm{L}}{\otimes} p_{*} \Lambda=\mathrm{R} f_{!}\left(\mathrm{A} \stackrel{\mathbf{L}}{\otimes} f^{*} p_{*} \Lambda\right)
$$

(projection formula 4.4 .2 for $f$ ). But, we have trivially the base change for $p$, namely

$$
f^{*} p_{*}=\pi_{*} \phi^{*}
$$

Therefore, one gets

$$
\begin{aligned}
\mathrm{R} f_{!}\left(\mathrm{A} \stackrel{\mathrm{L}}{\otimes} f^{*} p_{*} \Lambda\right) & =\mathrm{R} f_{!}\left(\mathrm{A} \stackrel{\mathbf{L}}{\otimes} \pi_{*} \phi^{*} \Lambda\right) \\
& =\mathrm{R} f_{!} \pi_{*}\left(\pi^{*} \mathrm{~A} \stackrel{\mathrm{L}}{\otimes} \phi^{*} \Lambda\right) \text { projection for } \pi \\
& =p_{*} \phi_{!} \pi^{*} \mathrm{~A} \text { because } \pi_{*}=\pi_{!} \text {(5.2.1). }
\end{aligned}
$$

Applying $p^{*}$ gives the base change isomorphism.

Remark 5.3.1. One can prove [5.0.1.3, at least for A bounded below, more directly as follows. Start with $\mathrm{A}$ on $\mathscr{X}$ an injective complex. Because $\mathrm{R}^{0} f_{*} \mathrm{~A}_{i}$ is flasque, it is $\Gamma_{\mathscr{Y}^{\prime}}$-acyclic. Then, $p^{!} \mathrm{R} f_{*} \mathrm{~A}$ can be computed using the complex $\mathscr{H}_{\mathscr{Y}^{\prime}}^{0}\left(\mathrm{R}^{0} f_{*} \mathrm{~A}_{i}\right)$. On the other hand, $\pi^{!} \mathrm{A}$ can be computed by the complex $\mathscr{H}_{\mathscr{X}^{\prime}}^{0}\left(\mathrm{~A}_{i}\right)$ which is a flasque complex (formal, or [3], V.4.11). Therefore, the direct image by $\phi$ is just $\mathrm{R}^{0} \phi_{*} \mathscr{H}_{\mathscr{X}^{\prime}}^{0}\left(\mathrm{~A}_{i}\right)$. One is reduced to the formula

$$
\mathrm{R}^{0} \phi_{*} \mathscr{H}_{\mathscr{X}^{\prime}}^{0}=\mathscr{H}_{\mathscr{Y}^{\prime}}^{0}\left(\mathrm{R}^{0} f_{*}\right)
$$


5.4. Base change by a universal homeomorphism. If $p$ is a universal homeomorphism, then $p^{!}=p^{*}$ and $\pi^{!}=\pi^{*}$. Thus in this case [5.0.1.3 is equivalent to an isomorphism $p^{*} \mathrm{R} f_{*} \rightarrow$ $\mathrm{R} \phi_{*} \pi^{*}$. We define such a morphism by taking the usual base change morphism (adjunction).

Let $\mathrm{A} \in \mathrm{D}_{c}(\mathscr{X})$. Using a hypercover of $\mathscr{X}$ as in 5.1. one sees that to prove that the map $p^{*} \mathrm{R} f_{*} \mathrm{~A} \rightarrow \mathrm{R} \phi_{*} \pi^{*} \mathrm{~A}$ is an isomorphism it suffices to consider the case when $\mathscr{X}$ is a scheme. Furthermore, by the smooth base change formula already shown, it suffices to prove that this map is an isomorphism after making a smooth base change $\mathrm{Y} \rightarrow \mathscr{Y}$. We may therefore assume that $\mathscr{Y}$ is also a scheme in which case the result follows from the classical corresponding result for étale topology (see [1], IV.4.10).

5.5. Base change morphism in general. Before defining the base change morphism we need a general construction of strictly simplicial schemes and algebraic spaces.

Fix an algebraic stack $\mathscr{X}$. In the following construction all schemes and morphisms are assumed over $\mathscr{X}$ (so in particular products are taken over $\mathscr{X}$ ).

Let $\mathrm{X}$. be a strictly simplicial scheme, $[n] \in \Delta^{+}$an object, and $a: \mathrm{V} \rightarrow \mathrm{X}_{n}$ a surjective morphism. We then construct a strictly simplicial scheme $\mathrm{M}\left(\mathrm{X}_{\bullet}, a\right)$ (sometimes written $\mathrm{M}_{\mathscr{X}}\left(\mathrm{X}_{\bullet}, a\right)$ if we want to make clear the reference to $\mathscr{X})$ with a morphism $\mathrm{M}\left(\mathrm{X}_{\bullet}, a\right) \rightarrow \mathrm{X}_{\bullet}$ such that the following hold:

(i) For $i<n$ the morphism $\mathrm{M}\left(\mathrm{X}_{\bullet}, a\right)_{i} \rightarrow \mathrm{X}_{i}$ is an isomorphism.

(ii) $\mathrm{M}\left(\mathrm{X}_{\bullet}, a\right)_{n}$ is equal to $\mathrm{V}$ with the projection to $\mathrm{X}_{n}$ given by $a$.

The construction of $\mathrm{M}\left(\mathrm{X}_{\bullet}, a\right)$ is a standard application of the skeleton and coskeleton functors ([3], exp. Vbis). Let us review some of this because the standard references deal only with simplicial spaces whereas we consider strictly simplicial spaces.

To construct $\mathrm{M}\left(\mathrm{X}_{\bullet}, a\right)$, let $\Delta_{n}^{+} \subset \Delta^{+}$denote the full subcategory whose objects are the finite sets with cardinality $\leq n$. Denote by $\mathrm{Sch}_{n}^{\Delta_{n}^{\text {opp }}}$ the category of functors from $\Delta_{n}^{+ \text {opp }}$ to schemes (so $\mathrm{Sch}^{\Delta^{+o p p}}$ is the category of strictly simplicial schemes). Restriction from $\Delta^{+ \text {opp }}$ to $\Delta_{n}^{+ \text {opp }}$ defines a functor (the $n$-skeleton functor)

$$
\mathrm{Sq}_{n}: \mathrm{Sch}^{\Delta^{+\mathrm{opp}}} \rightarrow \mathrm{Sch}^{\Delta_{n}^{+\mathrm{opp}}}
$$

which has a right adjoint

$$
\operatorname{cosq}_{n}: \operatorname{Sch}^{\Delta_{n}^{+o p p}} \rightarrow \operatorname{Sch}^{\Delta^{+o p p}}
$$


called the $n$-th coskeleton functor. For $\mathrm{X}_{\bullet} \in \mathrm{Sch}_{n}^{\Delta_{n}^{+o p p}}$, the coskeleton $\operatorname{cosq}_{n} \mathrm{X}$ in degree $i$ is equal to

$$
\left(\operatorname{cosq}_{n} \mathrm{X}\right)_{i}=\lim _{\substack{[k] \rightarrow[i] \\ k \leq n}} \mathrm{X}_{k},
$$

where the limit is taken over the category of morphisms $[k] \rightarrow[i]$ in $\Delta^{+}$with $k \leq n$.

Note in particular that for $i \leq n$ we have $\left(\operatorname{cosq}_{n} \mathrm{X}\right)_{i}=\mathrm{X}_{i}$ since the category of morphisms $[k] \rightarrow[i]$ has an initial object id $:[i] \rightarrow[i]$.

Lemma 5.5.1. For any $\mathrm{X}_{\bullet} \in \mathrm{Sch}^{\Delta_{n}^{+o p p}}$ and $i>n$ the morphism

$$
\left(\operatorname{cosq}_{n} \mathrm{X}\right)_{i} \rightarrow\left(\operatorname{cosq}_{i-1} \mathrm{sq}_{i-1} \operatorname{cosq}_{n} \mathrm{X}\right)_{i}
$$

is an isomorphism.

Proof: Using the formula 5.5.0.3 the morphism can be identified with the natural map

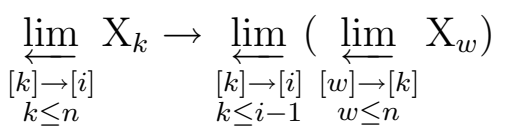

which is clearly an isomorphism.

Lemma 5.5.2. The functors $\mathrm{sq}_{n}$ and $\operatorname{cosq}_{n}$ commute with fiber products.

Proof: The functor $\mathrm{sq}_{n}$ commutes with fiber products by construction, and the functor $\operatorname{cosq}_{n}$ commutes with fiber products by adjunction.

To construct $\mathrm{M}\left(\mathrm{X}_{\bullet}, a\right)$, we first construct an object $\mathrm{M}^{\prime}\left(\mathrm{X}_{\bullet}, a\right) \in \mathrm{Sch}_{n}^{\Delta_{n}^{\text {opp }}}$. The restriction of $\mathrm{M}^{\prime}\left(\mathrm{X}_{\bullet}, a\right)$ to $\Delta_{n-1}^{+ \text {opp }}$ will be equal to $\mathrm{sq}_{n-1} \mathrm{X}$, and $\mathrm{M}^{\prime}\left(\mathrm{X}_{\bullet}, a\right)_{n}$ is defined to be $\mathrm{V}$. For $0 \leq j \leq n$ define $\delta_{j}: \mathrm{M}^{\prime}\left(\mathrm{X}_{\bullet}, a\right)_{n} \rightarrow \mathrm{M}^{\prime}\left(\mathrm{X}_{\bullet}, a\right)_{n-1}=\mathrm{X}_{n-1}$ to be the composite

$$
\mathrm{V} \stackrel{a}{\longrightarrow} \mathrm{X}_{n} \stackrel{\delta_{\mathrm{X}, j}}{\longrightarrow} \mathrm{X}_{n-1}
$$

where $\delta_{j, \mathrm{X}}$ denotes the map obtained from the strictly simplicial structure on $\mathrm{X}_{\bullet}$. There is an obvious morphism

$$
\left.\mathrm{M}^{\prime}\left(\mathrm{X}_{\bullet}, a\right) \rightarrow \mathrm{sq}_{n}\left(\mathrm{X}_{\bullet}\right) \text { inducing } \operatorname{cosq}_{n} \mathrm{M}^{\prime}\left(\mathrm{X}_{\bullet}, a\right)\right) \rightarrow \operatorname{cosq}_{n} \mathrm{sq}_{n} \mathrm{X}_{\bullet}
$$

We then define

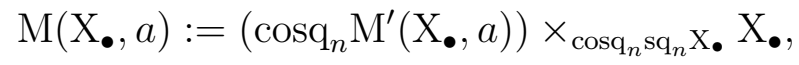


where the map $\mathrm{X}_{\bullet} \rightarrow \operatorname{cosq}_{n} \mathrm{sq}_{n} \mathrm{X}_{\bullet}$ is the adjunction morphism. The map $\mathrm{M}\left(\mathrm{X}_{\bullet}, a\right) \rightarrow \mathrm{X}_{\bullet}$ is defined to be the projection. The properties (i) and (ii) follow immediately from the construction.

Proposition 5.5.3. Let $\mathscr{X}$ be an algebraic stack and $\mathrm{X} \bullet \rightarrow \mathscr{X}$ a hypercover by schemes. Let $n$ be a natural number and $a: \mathrm{V} \rightarrow \mathrm{X}_{n}$ a surjection. Then $\mathrm{M}_{\mathscr{X}}\left(\mathrm{X}_{\bullet}, a\right) \rightarrow \mathscr{X}$ is also a hypercover. If $\mathrm{X}_{\bullet}$ is a smooth hypercover and $a$ is smooth and surjective, then $\mathrm{M}_{\mathscr{X}}\left(\mathrm{X}_{\bullet}, a\right)$ is also a smooth hypercover.

Proof: By definition of a hypercover, we must verify that for all $i$ the map

$$
\mathrm{M}\left(\mathrm{X}_{\bullet}, a\right)_{i} \rightarrow\left(\operatorname{cosq}_{i-1} \mathrm{sq}_{i-1} \mathrm{M}\left(\mathrm{X}_{\bullet}, a\right)\right)_{i}
$$

is surjective. Note that this is immediate for $i \leq n$. For $i>n$ we compute

$$
\begin{aligned}
\left(\operatorname{cosq}_{i-1} \mathrm{sq}_{i-1} \mathrm{M}\left(\mathrm{X}_{\bullet}, a\right)\right)_{i} & \simeq\left(\operatorname { c o s q } _ { i - 1 } \mathrm { sq } _ { i - 1 } \left(\operatorname{cosq}_{n} \mathrm{M}^{\prime}\left(\mathrm{X}_{\bullet}, a\right) \times_{\left.\left.\operatorname{cosq}_{n} \mathrm{sq}_{n} \mathrm{x}_{\bullet} \mathrm{X}_{\bullet}\right)\right)_{i}}\right.\right. \\
& \simeq\left(\operatorname{cosq}_{i-1} \mathrm{sq}_{i-1}\left(\operatorname{cosq}_{n} \mathrm{M}^{\prime}\left(\mathrm{X}_{\bullet}, a\right)\right)\right)_{i} \times\left(\operatorname{cosq}_{i} \mathrm{sq}_{i-1} \operatorname{cosq}_{n} \mathrm{sq}_{n} \mathrm{x}_{\bullet}\right)_{i}\left(\operatorname{cosq}_{i-1} \mathrm{sq}_{i-1} \mathrm{X}_{\bullet}\right)_{i} \\
& \simeq\left(\operatorname{cosq}_{n} \mathrm{M}^{\prime}\left(\mathrm{X}_{\bullet}, a\right)\right)_{i} \times\left(\operatorname{cosq}_{n} \mathrm{sq}_{n} \mathrm{x}_{\bullet}\right)_{i}\left(\operatorname{cosq}_{i-1} \mathrm{sq}_{i-1} \mathrm{X}_{\bullet}\right)_{i}
\end{aligned}
$$

Here the second isomorphism is because $\mathrm{sq}_{n}$ and $\operatorname{cosq}_{n}$ commute with products, and the third isomorphism is by 5.5.1. Hence it suffices to show that the natural map

$$
\mathrm{X}_{i} \rightarrow\left(\operatorname{cosq}_{i-1} \mathrm{sq}_{i-1} \mathrm{X}_{\bullet}\right)_{i}
$$

is surjective, which is true since $\mathrm{X}_{\bullet}$ is a hypercover. This also proves that if $\mathrm{X}_{\bullet}$ is a smooth hypercover and $a$ is smooth, then $\mathrm{M}_{\mathscr{X}}\left(\mathrm{X}_{\bullet}, a\right)$ is a smooth hypercover.

The construction of $\mathrm{M}_{\mathscr{X}}\left(\mathrm{X}_{\bullet}, a\right)$ is functorial. Precisely, let $f: \mathscr{X} \rightarrow \mathscr{Y}$ be a morphism of algebraic stacks, $\mathrm{X}_{\bullet} \rightarrow \mathscr{X}$ a strictly simplicial scheme over $\mathscr{X}, \mathrm{Y}_{\bullet} \rightarrow \mathscr{Y}$ a strictly simplicial scheme over $\mathscr{Y}$, and $f_{\bullet}: \mathrm{X}_{\bullet} \rightarrow \mathrm{Y}_{\bullet}$ a morphism over $f$. Then for any commutative diagram of schemes

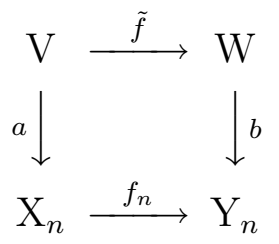

there is an induced morphism of strictly simplicial schemes $\mathrm{M}_{\mathscr{X}}\left(\mathrm{X}_{\bullet}, a\right) \rightarrow \mathrm{M}_{\mathscr{Y}}\left(\mathrm{Y}_{\bullet}, b\right)$ over $f_{\bullet}$.

Proposition 5.5.4. Let $f: \mathscr{X} \rightarrow \mathscr{Y}$ be a morphism of finite type between algebraic S-stacks locally of finite type. Then there exists smooth hypercovers $p: \mathrm{X}_{\bullet} \rightarrow \mathscr{X}$ and $q: \mathrm{Y}_{\bullet} \rightarrow \mathscr{Y}$ by 
schemes and a commutative diagram

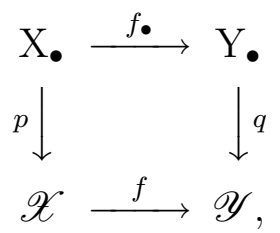

where each morphism $f_{n}: \mathrm{X}_{n} \rightarrow \mathrm{Y}_{n}$ is a closed immersion.

Proof: We construct inductively hypercovers $\mathrm{X}_{\bullet}^{(n)} \rightarrow \mathscr{X}$ and $\mathrm{Y}_{\bullet}^{(n)} \rightarrow \mathscr{Y}$ and a commutative diagram

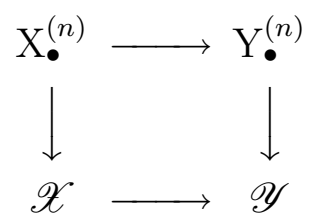

together with a commutative diagram

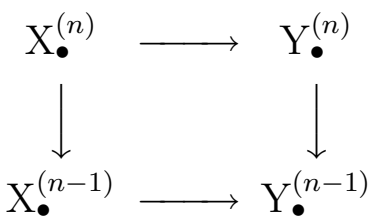

over $f$. We further arrange so that the following hold:

(i) For $i<n$ the maps $\mathrm{X}_{i}^{(n)} \rightarrow \mathrm{X}_{i}^{(n-1)}$ and $\mathrm{Y}_{i}^{(n)} \rightarrow \mathrm{Y}_{i}^{(n-1)}$ are isomorphisms.

(ii) For $i \leq n$ the maps $\mathrm{X}_{i}^{(n)} \rightarrow \mathrm{Y}_{i}^{(n)}$ are closed immersions.

This suffices for we can then take $\mathrm{X}_{\bullet}=\lim _{\longleftarrow} \mathrm{X}^{(n)}$ and $\mathrm{Y}_{\bullet}=\lim _{\longleftarrow} \mathrm{Y}^{(n)}$.

For the base case $n=0$, choose any 2 -commutative diagram

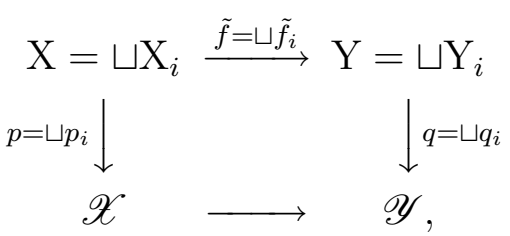

with $p_{i}$ and $q_{i}$ smooth, surjective, and of finite type, and $\mathrm{X}_{i}$ and $\mathrm{Y}_{i}$ affine schemes. Then $\tilde{f}_{i}$ are also of finite type, so there exists a closed immersion $\mathrm{X}_{i} \hookrightarrow \mathbb{A}_{\mathrm{Y}_{i}}^{r_{i}}$ for some integer $r$ over $\mathrm{X}_{i} \rightarrow \mathrm{Y}_{i}$. Replacing $\mathrm{Y}_{i}$ by $\mathbb{A}_{\mathrm{Y}_{i}}^{r_{i}}$ we may assume that $\tilde{f}$ is a closed immersions. We then obtain $\mathrm{X}_{\bullet}^{(0)} \rightarrow \mathrm{Y}_{\bullet}^{(0)}$ by taking the coskeletons of $p$ and $q$.

Now assume that $\mathrm{X}_{\bullet}^{(n-1)} \rightarrow \mathrm{Y}_{\bullet}^{(n-1)}$ has been constructed. Choose a commutative diagram

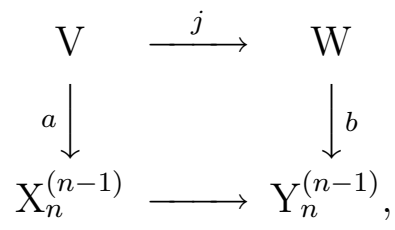


with $a$ and $b$ smooth and surjective, and $j$ a closed immersion. Then define $\mathrm{X}_{\bullet}^{(n)} \rightarrow \mathrm{Y}_{\bullet}^{(n)}$ to be

$$
\mathrm{M}_{\mathscr{X}}\left(\mathrm{X}_{\bullet}^{(n-1)}, a\right) \rightarrow \mathrm{M}_{\mathscr{Y}}\left(\mathrm{Y}_{\bullet}^{(n-1)}, b\right)
$$

Remark 5.5.5. The same argument used in the proof shows that for any commutative diagram

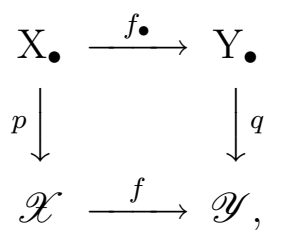

where $p$ and $q$ are smooth hypercovers, there exists a morphism of simplicial schemes $g: \widetilde{\mathrm{X}}_{\bullet} \rightarrow$ $\tilde{Y}_{\bullet}$ over $f_{\bullet}$ with each $g_{n}: \widetilde{\mathrm{X}}_{n} \rightarrow \widetilde{\mathrm{Y}}_{n}$ an immersion such that $\widetilde{\mathrm{X}}_{\bullet}$ (resp. $\tilde{\mathrm{Y}}_{\bullet}$ ) is a hypercover of $\mathscr{X}$ (resp. $\mathscr{Y}$ ). In other words, the category of diagrams 5.5.4.1 is connected.

Let $f: \mathscr{X} \rightarrow \mathscr{Y}$ be a morphism of algebraic stacks over $\mathrm{S}$. For $\mathrm{F} \in \mathrm{D}_{c}^{-}(\mathscr{X})$ we can compute $\mathrm{R} f_{!} \mathrm{F}$ as follows. Let $\mathrm{Y}_{\bullet} \rightarrow \mathscr{Y}$ be a smooth hypercover, and let $\pi: \mathscr{X}_{\mathrm{Y}} \rightarrow \mathscr{X}$ be the base change of $\mathscr{X}$ to $\mathrm{Y}_{\bullet}$. Let $f_{\bullet}: \mathscr{X}_{\mathrm{Y}_{\bullet}} \rightarrow \mathrm{Y}_{\bullet}$ be the projection. Let $\omega_{\mathscr{X}_{\mathrm{Y}}}$ denote the pullback of the dualizing sheaf $\Omega_{\mathscr{X}}$ to $\mathscr{X}_{\mathrm{Y}_{\bullet}}$, and let $\mathrm{D}_{\mathscr{X}_{\mathrm{Y}}}$ denote the functor $\mathscr{R} h o m\left(-, \omega_{\mathscr{X}_{\mathbf{Y}}}\right)$. Similarly let $\omega_{\mathrm{Y}_{\bullet}}$ denote the pullback of $\Omega_{\mathscr{Y}}$ to $\mathrm{Y}_{\bullet}$, and let $\mathrm{D}_{\mathrm{Y}_{\bullet}}$ denote $\mathscr{R} h o m\left(-, \omega_{\mathrm{Y}_{\bullet}}\right)$.

If $d_{n}$ (resp. $d_{n}^{\prime}$ ) denotes the relative dimension of $\mathrm{Y}_{n}$ over $\mathscr{Y}$ (resp. $\mathrm{Y}_{n}^{\prime}$ over $\mathscr{Y}^{\prime}$ ), then $d_{n}$ (resp. $d_{n}^{\prime}$ ) is also equal to the relative dimension of $\mathscr{X}_{\mathrm{Y}_{n}}$ over $\mathscr{X}$ (resp. $\mathscr{X}_{\mathrm{Y}_{n}^{\prime}}^{\prime}$ over $\mathscr{X}^{\prime}$ ). From 4.5.2 it follows that the restriction of $\omega_{\mathscr{X}_{\mathrm{Y}}}$ to $\mathscr{X}_{\mathrm{Y}_{n}}$ is canonically isomorphic to $\Omega_{\mathscr{X}_{\mathrm{Y}_{n}}}\left\langle-d_{n}\right\rangle$. Similarly the restriction of $\omega_{\mathrm{Y}}$. to $\mathrm{Y}_{n}$ is canonically isomorphic to $\Omega_{\mathrm{Y}_{n}}\left\langle-d_{n}\right\rangle$. Note that this combined with 3.5.7 shows that $\mathrm{D}_{\mathrm{Y}_{\bullet}} \circ \mathrm{D}_{\mathrm{Y}_{\bullet}}=\mathrm{id}\left(\right.$ resp. $\mathrm{D}_{\mathscr{X}_{\mathrm{Y}_{\bullet}}} \circ \mathrm{D}_{\mathscr{X}_{\mathrm{Y}_{\bullet}}}=\mathrm{id}$ ) on the category $\mathrm{D}_{c}\left(\mathrm{Y}_{\bullet}\right)\left(\operatorname{resp} . \mathrm{D}_{c}\left(\mathscr{X}_{\mathrm{Y}_{\bullet}}\right)\right)$.

For $\mathrm{F} \in \mathrm{D}_{c}(\mathscr{X})$, we can then consider

$$
\mathrm{D}_{\mathrm{Y} \bullet} \mathrm{R} f_{\bullet *} \mathrm{D}_{\mathscr{X}_{\mathrm{Y}}}\left(\pi^{*} \mathrm{~F}\right) \in \mathrm{D}\left(\mathrm{Y}_{\bullet}, \text { ét }\right) .
$$

The sheaf $\mathrm{D}_{\mathscr{X}_{\mathrm{Y}}}\left(\pi^{*} \mathrm{~F}\right)$ is just the restriction of $\mathrm{D}_{\mathscr{X}}(\mathrm{F})$ to $\mathscr{X}_{\mathrm{Y}}$. It follows from this that $\mathrm{R} f_{\bullet *} \mathrm{D}_{\mathscr{X}_{\mathrm{Y}}}\left(\pi^{*} \mathrm{~F}\right)$ is equal to the restriction of $\mathrm{R} f_{*} \mathrm{D}_{\mathscr{X}}(\mathrm{F})$ to $\mathrm{Y}_{\bullet}$, and this in turn implies that $\mathrm{D}_{\mathrm{Y}} \cdot \mathrm{R} f_{\bullet *} \mathrm{D}_{\mathscr{X}_{\mathrm{Y}}}\left(\pi^{*} \mathrm{~F}\right)$ is isomorphic to the restriction of $\mathrm{R} f_{!} \mathrm{F}$ to $\mathrm{Y}_{\bullet}$,ét. From this we conclude that $\mathrm{R} f_{!} \mathrm{F}$ is equal to the sheaf obtained from $\mathrm{D}_{\mathrm{Y}} \mathrm{R} f_{\bullet *} \mathrm{D}_{\mathscr{X}_{\mathrm{Y}}}\left(\pi^{*} \mathrm{~F}\right)$ and the equivalence of categories (2.2.6) $\mathrm{D}_{c}(\mathscr{Y}) \simeq \mathrm{D}_{c}\left(\mathrm{Y}_{\bullet}\right)$. 
Theorem 5.5.6. Let

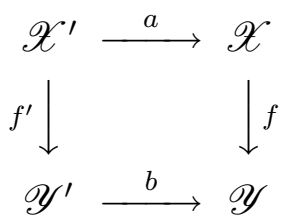

be a cartesian square of stacks over $\mathrm{S}$. Then there is a natural isomorphism of functors

$$
b^{*} \mathrm{R} f_{!} \rightarrow \mathrm{R} f_{!}^{\prime} a^{*}
$$

Proof: By 5.5.4 there exists a commutative diagram

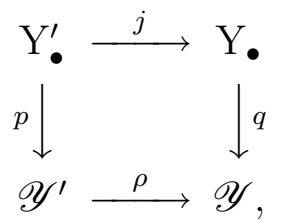

where $p$ and $q$ are smooth hypercovers and $j$ is a closed immersion.

Let $\mathscr{X}_{\mathrm{Y}_{\bullet}^{\prime}}^{\prime}$ denote the base change $\mathscr{X}^{\prime} \times_{\mathscr{Y}} \mathrm{Y}_{\bullet}^{\prime}$ and $\mathscr{X}_{\mathrm{Y}}$ the base change $\mathscr{X} \times \mathscr{Y} \mathrm{Y}_{\bullet}$. Then there is a cartesian diagram

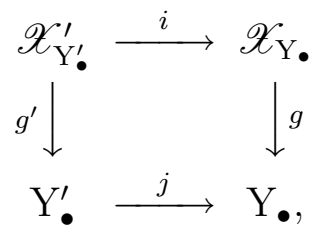

where $i$ and $j$ are closed immersions.

As before let $\omega_{\mathscr{X}_{\mathrm{Y}^{\prime}}^{\prime}}\left(\operatorname{resp} . \omega_{\mathscr{X}_{\mathrm{Y}}}, \omega_{\mathrm{Y}_{\bullet}^{\prime}}, \omega_{\mathrm{Y}}\right)$ denote the pullback of $\Omega_{\mathscr{X}^{\prime}}$ (resp. $\Omega_{\mathscr{X}}, \Omega_{\mathscr{Y}{ }^{\prime}}$, $\left.\Omega_{\mathscr{Y}}\right)$ to $\mathscr{X}_{\mathrm{Y}_{\bullet}^{\prime}}^{\prime}\left(\right.$ resp. $\left.\mathscr{X}_{\mathrm{Y}_{\bullet}}, \mathrm{Y}_{\bullet}^{\prime}, \mathrm{Y}_{\bullet}\right)$, and let $\mathrm{D}_{\mathscr{X}_{\mathrm{Y}_{\bullet}^{\prime}}^{\prime}}\left(\right.$ resp. $\left.\mathrm{D}_{\mathscr{X}_{\mathrm{Y}_{\bullet}}}, \mathrm{D}_{\mathrm{Y}_{\bullet}^{\prime}}, \mathrm{D}_{\mathrm{Y}_{\bullet}}\right)$ denote the functor $\mathscr{R h o m}\left(-, \omega_{\mathrm{X}_{\mathrm{Y}_{\bullet}^{\prime}}^{\prime}}\right)\left(\right.$ resp. $\left.\mathscr{R h o m}\left(-, \omega_{\mathscr{X}_{\mathrm{Y}}}\right), \mathscr{R h o m}\left(-, \omega_{\mathrm{Y}_{\bullet}^{\prime}}\right), \mathscr{R h o m}\left(-, \omega_{\mathrm{Y}_{\bullet}}\right)\right)$.

Lemma 5.5.7. Let $\mathscr{T}$ be a topos and $\Lambda$ a sheaf of rings in $\mathscr{T}$. Then for any $\mathrm{A}, \mathrm{B}, \mathrm{C} \in \mathrm{D}(\mathscr{T}, \Lambda)$ there is a canonical morphism

$$
\mathrm{A} \stackrel{\mathbf{L}}{\otimes} \mathscr{R h o m}(\mathrm{B}, \mathrm{C}) \rightarrow \mathscr{R h o m}(\mathscr{R} h o m(\mathrm{~A}, \mathrm{~B}), \mathrm{C}) .
$$

Proof: We have

$\operatorname{Rhom}(\mathrm{A} \stackrel{\mathrm{L}}{\otimes} \mathscr{R} h o m(\mathrm{~B}, \mathrm{C}), \mathscr{R} h o m(\mathscr{R} h o m(\mathrm{~A}, \mathrm{~B}), \mathrm{C})) \simeq \operatorname{Rhom}\left(\mathrm{A} \stackrel{\mathrm{L}}{\otimes} \mathscr{R} h o m(\mathrm{~B}, \mathrm{C})^{\mathrm{L}} \otimes \mathscr{R} h o m(\mathrm{~A}, \mathrm{~B}), \mathrm{C}\right)$.

Let

$$
a: \mathrm{A} \stackrel{\mathbf{L}}{\otimes} \mathscr{R} h o m(\mathrm{~A}, \mathrm{~B}) \rightarrow \mathrm{B}, \quad b: \stackrel{\mathrm{L}}{\otimes} \mathscr{R} h o m(\mathrm{~B}, \mathrm{C}) \rightarrow \mathrm{C}
$$


be the evaluation morphisms. Then the morphism

$$
\mathrm{A} \stackrel{\mathrm{L}}{\otimes} \mathscr{R} h o m(\mathrm{~B}, \mathrm{C}) \stackrel{\mathrm{L}}{\otimes} \mathscr{R} h o m(\mathrm{~A}, \mathrm{~B}) \stackrel{a}{\rightarrow} \mathrm{B} \stackrel{\mathrm{L}}{\otimes} \mathscr{R} h o m(\mathrm{~B}, \mathrm{C}) \stackrel{b}{\rightarrow} \mathrm{C}
$$

and the isomorphism 5.5 .7 .2 give the Lemma.

Let $\mathscr{F}$ denote the functor

$$
\mathrm{D}_{\mathrm{Y}_{\bullet}^{\prime}} j^{*} \mathrm{D}_{\mathrm{Y}_{\bullet}} g_{*} \mathrm{D}_{\mathscr{X}_{\bullet}} i_{*} \mathrm{D}_{\mathscr{X}_{\mathrm{Y}_{\bullet}^{\prime}}^{\prime}}: \mathrm{D}_{c}\left(\mathscr{X}_{\mathrm{Y}_{\bullet}^{\prime}}^{\prime}\right) \rightarrow \mathrm{D}\left(\mathrm{Y}_{\bullet}^{\prime}\right)
$$

Proposition 5.5.8. There is an isomorphism of functors $\mathscr{F} \simeq \mathrm{R} g_{*}^{\prime}$.

Proof: Consider first the functor $\mathscr{F}^{\prime}:=\mathscr{F} \circ g^{\prime *}$ and let $\mathrm{A} \in \mathrm{D}_{c}^{-}\left(\mathrm{Y}_{\bullet}^{\prime}\right)$. Then

$$
\begin{aligned}
& \mathscr{F}^{\prime}(\mathrm{A})=\mathrm{D}_{\mathrm{Y}_{\bullet}^{\prime}} j^{*} \mathrm{D}_{\mathrm{Y}_{\bullet}} g_{*} \mathscr{R h o m}\left(i_{*} \mathscr{R h o m}\left(g^{\prime *} \mathrm{~A}, \omega_{\mathscr{X}_{\mathrm{Y}_{\bullet}^{\prime}}^{\prime}}\right), \omega_{\mathscr{X}_{\mathrm{Y}_{\bullet}}}\right) \quad \text { (definition) } \\
& \simeq \mathrm{D}_{\mathrm{Y}_{\bullet}^{\prime}} j^{*} \mathrm{D}_{\mathrm{Y}_{\bullet}} g_{*} \mathscr{R h o m}\left(i_{*} \mathscr{R h o m}\left(i^{*} i_{*} g^{*} \mathrm{~A}, \omega_{\mathscr{X}_{\mathrm{Y}_{\bullet}^{\prime}}^{\prime}}\right), \omega_{\mathscr{X}_{\mathrm{Y}}}\right) \quad\left(i^{*} i_{*}=\mathrm{id}\right) \\
& \simeq \mathrm{D}_{\mathrm{Y}_{\bullet}^{\prime}} j^{*} \mathrm{D}_{\mathrm{Y}_{\bullet}} g_{*} \mathscr{R} h o m\left(\mathscr{R} h o m\left(i_{*} g^{\prime *} \mathrm{~A}, i_{*} \omega_{\mathscr{X}_{\mathrm{Y}_{\bullet}^{\prime}}^{\prime}}\right), \omega_{\mathscr{X}_{\mathrm{Y}_{\bullet}}}\right) \quad\left(\text { adjunction for }\left(i^{*}, i_{*}\right)\right) \\
& \leftarrow \mathrm{D}_{\mathrm{Y}_{\bullet}^{\prime}} j^{*} \mathrm{D}_{\mathrm{Y} \bullet} g_{*}\left(i_{*} g^{\prime *} \mathrm{~A} \stackrel{\mathrm{L}}{\otimes} \mathscr{R} h o m\left(i_{*} \omega_{\mathscr{X}_{\mathrm{Y}^{\prime}}^{\prime}}, \omega_{\mathscr{X}_{\mathrm{Y}}}\right)\right) \text { (15.5.7) } \\
& \simeq \mathrm{D}_{\mathrm{Y}_{\bullet}^{\prime}} j^{*} \mathrm{D}_{\mathrm{Y}_{\bullet}} g_{*}\left(g^{*} j_{*} \mathrm{~A} \stackrel{\mathrm{L}}{\otimes} \mathscr{R} h o m\left(i_{*} \omega_{\mathscr{X}_{\mathrm{Y}_{\bullet}^{\prime}}^{\prime}}, \omega_{\mathscr{X}_{\mathrm{Y}}}\right)\right) \quad\left(i_{*} g^{\prime *}=g^{*} j_{*} \text { by proper base change }\right) \\
& \simeq \mathrm{D}_{\mathrm{Y}_{\bullet}^{\prime}} j^{*} \mathrm{D}_{\mathrm{Y}_{\bullet}}\left(j_{*} \mathrm{~A} \stackrel{\mathrm{L}}{\otimes} \mathrm{R} g_{*} \mathscr{R} h o m\left(i_{*} \omega_{\mathscr{X}_{\mathrm{Y}_{\bullet}^{\prime}}^{\prime}}, \omega_{\mathscr{X}_{\mathrm{Y}}}\right)\right) \quad \text { (projection formula) } \\
& \simeq \mathscr{R h o m}\left(j^{*} \mathscr{R h o m}\left(j_{*} \mathrm{~A} \stackrel{\mathrm{L}}{\otimes} \operatorname{R} g_{*} \mathscr{R} h o m\left(i_{*} \omega_{\mathscr{X}_{\mathrm{Y}_{\bullet}^{\prime}}^{\prime}}, \omega_{\mathscr{X}_{\mathrm{Y}}}\right), \omega_{\mathrm{Y}_{\bullet}}\right), \omega_{\mathrm{Y}_{\bullet}^{\prime}}\right) \quad \text { (definition) } \\
& \simeq j^{*} j_{*} \mathscr{R h o m}\left(j^{*} \mathscr{R h o m}\left(j_{*} \mathrm{~A} \stackrel{\mathrm{L}}{\otimes} \mathrm{R} g_{*} \mathscr{R h o m}\left(i_{*} \omega_{\mathscr{X}_{\mathrm{Y}_{\bullet}^{\prime}}^{\prime}}, \omega_{\mathscr{X}_{\mathrm{Y}}}\right), \omega_{\mathrm{Y}_{\bullet}}\right), \omega_{\mathrm{Y}_{\bullet}^{\prime}}\right) \quad\left(j^{*} j_{*}=\mathrm{id}\right) \\
& \left.\simeq j^{*} \mathscr{R h o m}\left(\mathscr{R h o m}\left(j_{*} \mathrm{~A} \stackrel{\mathbf{L}}{\otimes} \mathrm{R} g_{*} \mathscr{R h o m}\left(i_{*} \omega_{\mathscr{X}_{\mathrm{Y}_{\bullet}^{\prime}}^{\prime}}, \omega_{\mathscr{X}_{\mathrm{Y}}}\right), \omega_{\mathrm{Y}_{\bullet}}\right), j_{*} \omega_{\mathrm{Y}_{\bullet}^{\prime}}\right) \quad \text { (adjunction for }\left(j^{*}, j_{*}\right)\right) \\
& \simeq j^{*} \mathscr{R h o m}\left(\mathscr{R h o m}\left(j_{*} \mathrm{~A}, \mathscr{R h o m}\left(\operatorname{R} g_{*} \mathscr{R h o m}\left(i_{*} \omega_{\mathscr{Y}_{\mathrm{Y}_{\bullet}^{\prime}}^{\prime}}, \omega_{\mathscr{X}_{\mathrm{Y}}}\right), \omega_{\mathrm{Y}_{\bullet}}\right)\right), j_{*} \omega_{\mathrm{Y}_{\bullet}^{\prime}}\right) \\
& \leftarrow \mathrm{A} \stackrel{\mathbf{L}}{\otimes} j^{*} \mathscr{R h o m}\left(\mathscr{R h o m}\left(\mathrm{R} g_{*} \mathscr{R h o m}\left(i_{*} \omega_{\mathscr{X}_{\mathrm{Y}_{\bullet}^{\prime}}^{\prime}}, \omega_{\mathscr{X}_{\mathrm{Y}}}\right), \omega_{\mathrm{Y}}\right), j_{*} \omega_{\mathrm{Y}_{\bullet}^{\prime}}\right) \text { (15.5.7). }
\end{aligned}
$$

The following Lemma therefore shows that there is a canonical morphism $\mathrm{A} \rightarrow \mathscr{F}^{\prime}(\mathrm{A})$ functorial in $\mathrm{A}$.

Lemma 5.5.9. For all $s \in \mathbb{Z}$ there is a canonical isomorphism

$$
\mathscr{H}^{s}\left(j^{*} \mathscr{R h o m}\left(\mathscr{R h o m}\left(\mathrm{R} g_{*} \mathscr{R h o m}\left(i_{*} \omega_{\mathscr{X}_{\mathrm{Y}_{\bullet}^{\prime}}^{\prime}}, \omega_{\mathscr{X}_{\mathrm{Y}_{\bullet}}}\right), \omega_{\mathrm{Y}_{\bullet}}\right), j_{*} \omega_{\mathrm{Y}_{\bullet}^{\prime}}\right) \simeq \mathrm{R}^{s} g_{*}^{\prime} \Lambda\right. \text {. }
$$

In particular, $\tau_{\leq 0} j^{*} \mathscr{R h o m}\left(\mathscr{R h o m}\left(\mathrm{R} g_{*} \mathscr{R h o m}\left(i_{*} \omega_{\mathscr{X}_{\mathrm{Y}_{\bullet}^{\prime}}^{\prime}}, \omega_{\mathscr{X}_{\mathrm{Y}_{\bullet}}}\right), \omega_{\mathrm{Y}_{\bullet}}\right), j_{*} \omega_{\mathrm{Y}_{\bullet}^{\prime}}\right) \simeq g_{*}^{\prime} \Lambda$, so the composite

$$
\Lambda \rightarrow g_{*}^{\prime} \Lambda \simeq \tau_{\leq 0} j^{*} \mathscr{R h o m}\left(\mathscr{R h o m}\left(\mathrm{R} g_{*} \mathscr{R h o m}\left(i_{*} \omega_{\mathscr{X}_{\mathrm{Y}_{\bullet}^{\prime}}^{\prime}}, \omega_{\mathscr{X}_{\mathrm{Y}}}\right), \omega_{\mathrm{Y}_{\bullet}}\right), j_{*} \omega_{\mathrm{Y}_{\bullet}^{\prime}}\right)
$$


induces a canonical morphism

$$
\Lambda \rightarrow j^{*} \mathscr{R h o m}\left(\mathscr{R h o m}\left(\mathrm{R} g_{*} \mathscr{R h o m}\left(i_{*} \omega_{\mathscr{X}_{\mathrm{Y}_{\bullet}^{\prime}}^{\prime}}, \omega_{\mathscr{X}_{\mathrm{Y}}}\right), \omega_{\mathrm{Y}_{\bullet}}\right), j_{*} \omega_{\mathrm{Y}_{\bullet}^{\prime}}\right)
$$

Proof: It suffices to construct such a canonical isomorphism over each $\mathrm{Y}_{n}^{\prime}$. Let $d_{n}$ (resp. $\left.d_{n}^{\prime}\right)$ denote the relative dimension of $\mathrm{Y}_{n}\left(\right.$ resp. $\left.\mathrm{Y}_{n}^{\prime}\right)$ over $\mathscr{Y}\left(\right.$ resp. $\left.\mathscr{Y}^{\prime}\right)$. Note that $d_{n}\left(\right.$ resp. $\left.d_{n}^{\prime}\right)$ is also equal to the relative dimension of $\mathscr{X}_{\mathrm{Y}_{n}}$ (resp. $\left.\mathscr{X}_{\mathrm{Y}_{n}^{\prime}}^{\prime}\right)$ over $\mathscr{X}$ (resp. $\mathscr{X}^{\prime}$ ). As mentioned above we therefore have

$$
\omega_{\mathscr{X}_{Y_{n}^{\prime}}^{\prime}} \simeq \Omega_{\mathscr{X}_{Y_{n}^{\prime}}^{\prime}}\left\langle-d_{n}^{\prime}\right\rangle, \quad \omega_{\mathscr{X}_{\mathrm{Y}_{n}}} \simeq \Omega_{\mathscr{X}_{\mathrm{Y}_{n}}}\left\langle-d_{n}\right\rangle, \quad \omega_{\mathrm{Y}_{n}} \simeq \Omega_{\mathrm{Y}_{n}}\left\langle-d_{n}\right\rangle, \quad \omega_{\mathrm{Y}_{n}^{\prime}} \simeq \Omega_{\mathrm{Y}_{n}^{\prime}}\left\langle-d_{n}^{\prime}\right\rangle .
$$

From this and an elementary manipulation using the identity

$$
\mathscr{R h o m}(\mathrm{A}\langle n\rangle, \mathrm{B}\langle m\rangle) \simeq \mathscr{R} h o m(\mathrm{~A}, \mathrm{~B})\langle m-n\rangle
$$

we get

$$
\begin{gathered}
j^{*} \mathscr{R h o m}\left(\mathscr{R h o m}\left(\operatorname{R} g_{*} \mathscr{R h o m}\left(i_{*} \omega_{\mathscr{X}_{\mathrm{Y}_{n}^{\prime}}^{\prime}}, \omega_{\mathscr{X}_{\mathrm{Y}_{n}}}\right), \omega_{\mathrm{Y}_{n}}\right), j_{*} \omega_{\mathrm{Y}_{n}^{\prime}}\right) \\
\simeq j^{*} \mathscr{R h o m}\left(\mathscr{R h o m}\left(\operatorname{R} g_{*} \mathscr{R h o m}\left(i_{*} \Omega_{\mathscr{X}_{\mathrm{Y}_{n}^{\prime}}^{\prime}}\left\langle-d_{n}^{\prime}\right\rangle, \Omega_{\mathscr{X}_{\mathrm{Y}_{n}}}\left\langle-d_{n}\right\rangle\right), \Omega_{\mathrm{Y}_{n}}\left\langle-d_{n}\right\rangle\right), j_{*} \Omega_{\mathrm{Y}_{n}^{\prime}}\left\langle-d_{n}^{\prime}\right\rangle\right) \\
\simeq \quad j^{*} \mathscr{R h o m}\left(\mathscr{R h o m}\left(\operatorname{Rg}_{*} \mathscr{R h o m}\left(i_{*} \Omega_{\mathscr{X}_{\mathrm{Y}_{n}^{\prime}}^{\prime}}, \Omega_{\mathscr{X}_{\mathrm{Y}_{n}}}\right), \Omega_{\mathrm{Y}_{n}}\right), j_{*} \Omega_{\mathrm{Y}_{n}^{\prime}}\right) .
\end{gathered}
$$

We then get

$$
\begin{aligned}
\mathscr{R h o m}\left(i_{*} \Omega_{\mathscr{X}_{\mathrm{Y}_{n}^{\prime}}^{\prime}}, \Omega_{\mathscr{X}_{\mathrm{Y}_{n}}}\right) & \simeq \mathscr{R h o m}\left(i_{1} \Omega_{\mathscr{X}_{\mathrm{Y}_{n}^{\prime}}^{\prime}}, \Omega_{\mathscr{X}_{\mathrm{Y}_{n}}}\right) \\
& \simeq i_{*} \mathscr{R h o m}\left(\Omega_{\mathscr{X}_{\mathrm{Y}_{n}^{\prime}}^{\prime}}, i^{!} \Omega_{\mathscr{X}_{\mathrm{Y}_{n}}}\right) \\
& \simeq i_{*} \mathscr{R h o m}\left(\Omega_{\mathscr{X}_{\mathrm{Y}_{n}^{\prime}}^{\prime}}, \Omega_{\mathscr{X}_{\mathrm{Y}_{n}^{\prime}}^{\prime}}\right) \quad\left(i \cdot \Omega_{\mathscr{X}_{\mathrm{Y}_{n}}}=\Omega_{\mathscr{X}_{\mathrm{Y}_{n}^{\prime}}^{\prime}}\right) \\
& \simeq i_{*} \Lambda \text { (3.5.7). }
\end{aligned}
$$

Therefore 5.5.9.1 is equal to

$$
j^{*} \mathscr{R h o m}\left(\mathscr{R h o m}\left(\mathrm{R}_{*} i_{*} \Lambda, \Omega_{\mathrm{Y}_{n}}\right), j_{*} \Omega_{\mathrm{Y}_{n}^{\prime}}\right) \simeq j^{*} \mathscr{R h o m}\left(\mathscr{R h o m}\left(j_{*} \operatorname{R} g_{*}^{\prime} \Lambda, \Omega_{\mathrm{Y}_{n}}\right), j_{*} \Omega_{\mathrm{Y}_{n}^{\prime}}\right) \text {. }
$$

Then

$$
\begin{aligned}
j^{*} \mathscr{R h o m}\left(\mathscr{R h o m}\left(j_{*} \operatorname{R} g_{*}^{\prime} \Lambda, \Omega_{\mathrm{Y}_{n}}\right), j_{*} \Omega_{\mathrm{Y}_{n}^{\prime}}\right) & \simeq j^{*} \mathscr{R h o m}\left(j_{*} \mathscr{R h o m}\left(\mathrm{R} g_{*}^{\prime} \Lambda, j^{\prime} \Omega_{\mathrm{Y}_{n}}\right), j_{*} \Omega_{\mathrm{Y}_{n}^{\prime}}\right) \\
& \simeq j^{*} j_{*} \mathscr{R h o m}\left(j^{*} j_{*} \mathscr{R h o m}\left(\mathrm{R} g_{*}^{\prime} \Lambda, \Omega_{\mathrm{Y}_{n}^{\prime}}\right), \Omega_{\mathrm{Y}_{n}^{\prime}}\right) \\
& \simeq \mathscr{R h o m}\left(\mathscr{R h o m}\left(\mathrm{R}_{*}^{\prime} \Lambda, \Omega_{\mathrm{Y}_{n}^{\prime}}\right), \Omega_{\mathrm{Y}_{n}^{\prime}}\right) \quad\left(j^{*} j_{*}=\mathrm{id}\right) \\
& \simeq \operatorname{R} g_{*}^{\prime} \Lambda
\end{aligned}
$$


The functor id $\rightarrow \mathscr{F}^{\prime}$ induces for any $\mathrm{A} \in \mathrm{D}_{c}\left(\mathrm{Y}_{\bullet}^{\prime}\right)$ and $\mathrm{B} \in \mathrm{D}_{c}\left(\mathscr{X}_{\mathrm{Y}_{\bullet}^{\prime}}^{\prime}\right)$ a morphism $\mathscr{R h o m}\left(\mathrm{A}, \mathrm{R} g_{*}^{\prime} \mathrm{B}\right) \rightarrow \mathscr{R h o m}\left(\mathrm{A}, \mathscr{F}^{\prime}\left(\mathrm{R} g_{*}^{\prime} \mathrm{B}\right)\right) \simeq \mathscr{R h o m}\left(\mathrm{A}, \mathscr{F}\left(g^{\prime *} \operatorname{Rg}_{*}^{\prime} \mathrm{B}\right)\right) \rightarrow \mathscr{R h o m}(\mathrm{A}, \mathscr{F}(\mathrm{B}))$ where the last morphism is induced by adjunction $g^{\prime *} \mathrm{R} g_{*}^{\prime} \mathrm{B} \rightarrow \mathrm{B}$. This map is functorial in A, so by Yoneda's Lemma we get a canonical morphism $\mathrm{R} g_{*}^{\prime} \mathrm{B} \rightarrow \mathscr{F}(\mathrm{B})$. To prove 5.5 .8 we show that this map is an isomorphism for all $\mathrm{B} \in \mathrm{D}_{c}\left(\mathscr{X}_{\mathrm{Y}_{\bullet}^{\prime}}^{\prime}\right)$.

For this we can restrict the map to any $\mathscr{X}_{\mathrm{Y}_{n}^{\prime}}^{\prime}$. Noting that the shifts and Tate twists cancel as in 5.5.9.1, we get

$$
\begin{aligned}
\left.\mathscr{F}(\mathrm{B})\right|_{\mathrm{Y}_{n}^{\prime}} & \simeq \mathscr{R h o m}\left(j^{*} \mathscr{R h o m}\left(\mathrm{R} g_{*} \mathscr{R h o m}\left(i_{*} \mathscr{R h o m}\left(\mathrm{B}, \Omega_{\mathscr{X}_{\mathrm{Y}_{n}^{\prime}}^{\prime}}\right), \Omega_{\mathscr{X}_{\mathrm{Y}_{n}}}\right), \Omega_{\mathrm{Y}_{n}}\right), \Omega_{\mathrm{Y}_{n}^{\prime}}\right) \\
& \simeq \mathscr{R h o m}\left(j^{*} \mathscr{R h o m}\left(\mathrm{R} g_{*} i_{*} \mathscr{R h o m}\left(\mathscr{R} h o m\left(\mathrm{~B}, \Omega_{\mathscr{X}_{\mathrm{Y}_{n}^{\prime}}^{\prime}}\right), \mathrm{R} i^{!} \Omega_{\mathscr{X}_{\mathrm{Y}_{n}}}\right), \Omega_{\mathrm{Y}_{n}}\right), \Omega_{\mathrm{Y}_{n}^{\prime}}\right) \quad \text { (4.3.2) } \\
& \simeq \mathscr{R h o m}\left(j^{*} \mathscr{R h o m}\left(j_{*} \mathrm{R} g_{*}^{\prime} \mathrm{B}, \Omega_{\mathrm{Y}_{n}}\right), \Omega_{\mathrm{Y}_{n}^{\prime}}\right) \quad\left(\mathrm{R} i^{!} \Omega_{\mathscr{X}_{\mathrm{Y}_{n}}}=\Omega_{\mathscr{X}_{\mathrm{Y}_{n}^{\prime}}^{\prime}} \text { 3.5.7 and } j_{*} \mathrm{R} g_{*}^{\prime}=\mathrm{R} g_{*} i_{*}\right) \\
& \simeq \mathscr{R h o m}\left(j^{*} j_{*} \mathscr{R h o m}\left(\mathrm{R} g_{*}^{\prime} \mathrm{B}, \mathrm{R} j^{\prime} \Omega_{\mathrm{Y}_{n}}\right), \Omega_{\mathrm{Y}_{n}^{\prime}}\right) \quad(4.3 .2) \\
& \simeq \mathscr{R h o m}\left(\mathscr{R} h o m\left(\mathrm{R} g_{*}^{\prime} \mathrm{B}, \Omega_{\mathrm{Y}_{n}^{\prime}}\right), \Omega_{\mathrm{Y}_{n}^{\prime}}\right) \quad\left(j^{*} j_{*}=\mathrm{id}, j^{!} \Omega_{\mathrm{Y}_{n}}=\Omega_{\mathrm{Y}_{n}^{\prime}}\right) \\
& \simeq \operatorname{R} g_{*}^{\prime} \mathrm{B} \text { (3.5.7). }
\end{aligned}
$$

We leave to the reader the task of verifying that this isomorphism agrees with the map obtained by restriction from the morphism $\mathscr{F}(\mathrm{B}) \rightarrow \mathrm{R} g_{*}^{\prime} \mathrm{B}$ constructed above, thereby completing the proof of 5.5 .8

Let $\pi: \mathscr{X}_{\mathrm{Y}_{\bullet}} \rightarrow \mathscr{X}$ (resp. $\pi^{\prime}: \mathscr{X}_{\mathrm{Y}_{\bullet}^{\prime}}^{\prime} \rightarrow \mathscr{X}^{\prime}$ ) denote the projection. The isomorphism $\mathscr{F} \simeq \mathrm{R} g_{*}^{\prime}$ induces a morphism of functors

$$
\begin{aligned}
& j^{*} \mathrm{D}_{\mathrm{Y}} \cdot \mathrm{R}_{*} \mathrm{D}_{\mathscr{X}_{\mathrm{Y}}} \quad \rightarrow \quad j^{*} \mathrm{D}_{\mathrm{Y}} \cdot \mathrm{R}_{*} \mathrm{D}_{\mathscr{X}_{\mathrm{Y}}} i_{*} i^{*} \quad\left(\mathrm{id} \rightarrow i_{*} i^{*}\right) \\
& \simeq \mathrm{D}_{\mathrm{Y}_{\bullet}^{\prime}} \mathrm{D}_{\mathrm{Y}_{\bullet}^{\prime}} j^{*} \mathrm{D}_{\mathrm{Y}} \mathrm{R}_{*} \mathrm{D}_{\mathscr{X}_{\mathrm{Y}}} i_{*} \mathrm{D}_{\mathscr{X}_{\mathrm{Y}_{\bullet}^{\prime}}^{\prime}} \mathrm{D}_{\mathscr{X}_{\mathrm{Y}^{\prime}}^{\prime}} i^{*} \\
& \simeq \quad \mathrm{D}_{\mathrm{Y}_{\bullet}^{\prime}} \mathscr{F} \mathrm{D}_{\mathscr{X}_{\mathrm{Y}^{\prime}}^{\prime}} i^{*} \quad \text { (definition) } \\
& \simeq \quad \mathrm{D}_{\mathrm{Y}_{\bullet}^{\prime}} \mathrm{R}_{*}^{\prime} \mathrm{D}_{\mathscr{X}_{\mathrm{Y}^{\prime}}^{\prime}} i^{*} \text { (5.5.8). }
\end{aligned}
$$

This map induces a morphism

$$
\begin{aligned}
& \rho^{*} \mathrm{R} f_{!} \simeq \rho^{*} \mathrm{R} q_{*} \mathrm{D}_{\mathrm{Y}} \cdot \mathrm{R} g_{*} \mathrm{D}_{\mathscr{X}_{\mathrm{Y}}} \pi^{*} \quad \text { (cohomological descent) } \\
& \rightarrow \mathrm{R} p_{*} j^{*} \mathrm{D}_{\mathrm{Y}_{\bullet}} \mathrm{R} g_{*} \mathrm{D}_{\mathscr{X}_{\mathbf{Y}}} \pi^{*} \quad \text { (base change morphism) } \\
& \rightarrow \quad \mathrm{R} p_{*} \mathrm{D}_{\mathrm{Y}_{\bullet}^{\prime}} \mathrm{R} g_{*}^{\prime} \mathrm{D}_{\mathscr{X}_{\mathrm{Y}_{\bullet}^{\prime}}^{\prime}} i^{*} \pi^{*} \quad \text { (5.5.9.2) } \\
& \simeq \quad \mathrm{R} p_{*} \mathrm{D}_{\mathrm{Y}_{\bullet}^{\prime}} \mathrm{R} g_{*}^{\prime} \mathrm{D}_{\mathscr{X}_{\mathrm{Y}_{\bullet}^{\prime}}^{\prime}} \pi^{\prime *} a^{*} \quad\left(i^{*} \pi^{*}=\pi^{\prime *} a^{*}\right) \\
& \simeq \quad \mathrm{R} f_{!}^{\prime} a^{*} \text { (cohomological descent). }
\end{aligned}
$$

which we call the base change morphism. 
By construction this morphism is compatible with smooth base change on $\mathscr{Y}$ and $\mathscr{Y}^{\prime}$. It follows that in order to verify that 5.5.9.3 is an isomorphism it suffices to consider the case when $\mathscr{Y}^{\prime}$ and $\mathscr{Y}$ are schemes. Furthermore, by construction if $\mathrm{X}_{\bullet} \rightarrow \mathscr{X}$ is a smooth hypercover and $\mathrm{X}^{\prime}$ the base change to $\mathscr{Y}^{\prime}$, then the base change arrow 5.5.9.3 is compatible with the spectral sequences 4.8.1. It follows that to verify that 5.5.9.3 is an isomorphism it suffices to consider the case of schemes which is 4], XVII, 5.2.6. Finally the independence of the choices follows by a standard argument from 5.5.5. This completes the proof of 5.5.6.

5.6. Equivalence of different definitions of base change morphism. In this subsection we show that the base changed morphism defined in the previous subsection agrees with the morphism defined earlier for smooth morphisms, immersions, and universal homeomorphisms.

5.6.1. The case when $\rho$ is smooth. Choose a diagram as in 5.5.6.3, and let $d$ denote the locally constant function on $\mathscr{Y}^{\prime}$ which is the relative dimension of $\rho$. For any morphism $\mathscr{Z} \rightarrow \mathscr{Y}^{\prime}$ we also write $d$ for the pullback of the function $d$ to $\mathscr{Z}$. Note that

$$
j^{*} \omega_{\mathrm{Y}_{\bullet}} \simeq \omega_{\mathrm{Y}_{\bullet}^{\prime}}\langle-d\rangle, \quad i^{*} \omega_{\mathscr{X}_{\mathrm{Y}_{\bullet}}} \simeq \omega_{\mathscr{X}_{\mathrm{Y}_{\bullet}^{\prime}}^{\prime}}\langle-d\rangle
$$

Lemma 5.6.2. For any $\mathrm{A} \in \mathrm{D}_{c}\left(\mathscr{X}_{\mathrm{Y}_{\bullet}}\right)$ (resp. $\mathrm{B} \in \mathrm{D}_{c}\left(\mathrm{Y}_{\bullet}\right)$ ) there is a natural isomorphism $\mathrm{D}_{\mathscr{X}_{\mathrm{Y}_{\bullet}^{\prime}}^{\prime}}\left(i^{*} \mathrm{~A}\langle d\rangle\right) \simeq i^{*} \mathrm{D}_{\mathscr{X}_{\bullet}}(\mathrm{A})\left(\operatorname{resp} . \mathrm{D}_{\mathrm{Y}_{\bullet}^{\prime}} j^{*}(\mathrm{~B}\langle d\rangle) \simeq j^{*} \mathrm{D}_{\mathrm{Y}}(\mathrm{B})\right)$.

Proof: Consider the natural map

$$
\begin{aligned}
i^{*} \mathscr{R h o m}\left(\mathrm{A}, \omega_{\mathscr{X}_{\mathrm{Y}_{\bullet}}}\right) & \rightarrow \mathscr{R h o m}\left(i^{*} \mathrm{~A}, i^{*} \omega_{\mathscr{X}_{\mathrm{Y}_{\bullet}}}\right) \\
& \simeq \mathscr{R h o m}\left(i^{*} \mathrm{~A}, \omega_{\mathscr{X}_{\mathrm{Y}^{\prime}}^{\prime}}\right)\langle-d\rangle \\
& \simeq \mathscr{R h o m}\left(i^{*} \mathrm{~A}\langle d\rangle, \omega_{\mathscr{X}_{\mathrm{Y}_{\bullet}^{\prime}}^{\prime}}\right) .
\end{aligned}
$$

We claim that this map is an isomorphism. This can be verified over each $\mathscr{X}_{\mathrm{Y}_{n}^{\prime}}^{\prime}$. Let $\pi_{n}$ : $\mathscr{X}_{\mathrm{Y}_{n}} \rightarrow \mathscr{X}$ (resp. $\pi_{n}^{\prime}: \mathscr{X}_{\mathrm{Y}_{n}^{\prime}}^{\prime} \rightarrow \mathscr{X}^{\prime}$ ) be the projection. By the equivalence of triangulated categories $\mathrm{D}_{c}\left(\mathscr{X}_{\mathrm{Y}_{\bullet}}\right) \simeq \mathrm{D}_{c}(\mathscr{X})$, there exists an object $\mathrm{A}^{\prime} \in \mathrm{D}_{c}(\mathscr{X})$ so that the restriction of $\mathrm{A}$ to $\mathscr{X}_{\mathrm{Y}_{n}}$ is isomorphic to $\pi_{n}^{*} \mathrm{~A}^{\prime}$. The morphism 5.6.2.1 is then identified with the isomorphism

$$
\begin{aligned}
i^{*} \mathscr{R h o m}\left(\mathrm{A}, \omega_{\mathscr{X}_{\bullet}}\right) & \simeq i^{*} \pi_{n}^{*} \mathscr{R h o m}\left(\mathrm{A}^{\prime}, \Omega_{\mathscr{X}}\right) \\
& \simeq \pi_{n}^{\prime *} \rho^{*} \mathscr{R h o m}\left(\mathrm{A}^{\prime}, \Omega_{\mathscr{X}}\right) \\
& \simeq \pi_{n}^{* *} \mathscr{R h o m}\left(\rho^{*} \mathrm{~A}^{\prime}, \rho^{*} \Omega_{\mathscr{X}}\right) \\
& \simeq \mathscr{R h o m}\left(\pi_{n}^{*} \rho^{*} \mathrm{~A}^{\prime}, \pi_{n}^{*} \rho^{*} \Omega_{\mathscr{X}}\right) \\
& \simeq \mathscr{R} h o m\left(i^{*} \mathrm{~A}\langle d\rangle, \omega_{\mathscr{X}_{\mathrm{Y}_{\bullet}^{\prime}}^{\prime}}\right) .
\end{aligned}
$$

The same argument proves the statement $\mathrm{D}_{\mathrm{Y}_{\bullet}^{\prime}} j^{*}(\mathrm{~B}\langle d\rangle) \simeq j^{*} \mathrm{D}_{\mathrm{Y}}(\mathrm{B})$. 
For any $\mathrm{A} \in \mathrm{D}_{c}\left(\mathrm{Y}_{\bullet}\right)$, let $\alpha_{\mathrm{A}}$ denote the isomorphism

$$
\begin{aligned}
j^{*} \mathrm{~A}\langle d\rangle & \simeq j^{*} \mathrm{D}_{\mathrm{Y}_{\bullet}} \mathrm{D}_{\mathrm{Y}}(\mathrm{A})\langle d\rangle \\
& \simeq \mathrm{D}_{\mathrm{Y}_{\bullet}^{\prime}} j^{*} \mathrm{D}_{\mathrm{Y}_{\bullet}}(\mathrm{A}) .
\end{aligned}
$$

For $\mathrm{B} \in \mathrm{D}_{c}\left(\mathscr{X}_{\mathrm{Y}}\right)$ let $\beta_{\mathrm{B}}$ denote the isomorphism

$$
\begin{aligned}
i^{*} \mathrm{~B}\langle d\rangle & \simeq i^{*} \mathrm{D}_{\mathscr{X}_{\mathrm{Y}}} \mathrm{D}_{\mathscr{X}_{\mathrm{Y}}}(\mathrm{B}) \\
& \simeq \mathrm{D}_{\mathscr{X}_{\mathrm{Y}_{\bullet}^{\prime}}^{\prime}} i^{*} \mathrm{D}_{\mathscr{X}_{\mathrm{Y}}}(\mathrm{B})
\end{aligned}
$$

Also for $\mathrm{C} \in \mathrm{D}_{c}\left(\mathscr{X}_{\mathrm{Y}_{\bullet}^{\prime}}^{\prime}\right)$ let $\gamma_{\mathrm{C}}$ be the isomorphism

$$
\begin{aligned}
\mathrm{C}\langle-d\rangle & \simeq \mathrm{D}_{\mathscr{X}_{\mathrm{Y}_{\bullet}^{\prime}}^{\prime}} \mathrm{D}_{\mathscr{X}_{\mathrm{Y}_{\bullet}^{\prime}}^{\prime}}(\mathrm{C}\langle-d\rangle) \\
& \simeq \mathrm{D}_{\mathscr{X}_{\mathrm{Y}_{\bullet}^{\prime}}^{\prime}} i^{*} i_{*} \mathrm{D}_{\mathscr{X}_{\mathrm{Y}_{\bullet}^{\prime}}^{\prime}}(\mathrm{C}\langle-d\rangle) \\
& \simeq i^{*} \mathrm{D}_{\mathscr{X}_{\mathrm{Y}_{\bullet}}} i_{*} \mathrm{D}_{\mathscr{X}_{\mathrm{Y}_{\bullet}^{\prime}}^{\prime}}(\mathrm{C}),
\end{aligned}
$$

and let $\gamma^{\prime}: \mathrm{D}_{\mathscr{X}_{\bullet}} i_{*} \mathrm{D}_{\mathscr{X}_{\mathrm{Y}^{\prime}}^{\prime}}(\mathrm{C}) \rightarrow i_{*} \mathrm{C}\langle-d\rangle$ denote the map obtained by adjunction. This map also induces for every $\mathrm{E} \in \dot{\mathrm{D}}_{c}\left(\mathscr{X}_{\mathrm{Y}}\right)$ a morphism $\delta_{\mathrm{E}}$ given by

$$
\mathrm{D}_{\mathscr{X}_{\mathrm{Y}}} i_{*} i^{*} \mathrm{D}_{\mathscr{X}_{\mathrm{Y}}}(\mathrm{E}) \longrightarrow \mathrm{D}_{\mathscr{X}_{\mathrm{Y}}} i_{*} \mathrm{D}_{\mathscr{X}_{\mathrm{Y}_{\bullet}^{\prime}}^{\prime}}\left(i^{*} \mathrm{E}\right)\langle d\rangle \stackrel{\gamma^{\prime}}{\longrightarrow} i_{*} i^{*} \mathrm{E}
$$

The map $\alpha_{\mathrm{A}}$ is a special case of a more general class of morphisms. For $\mathrm{A}, \mathrm{M} \in \mathrm{D}_{c}\left(\mathrm{Y}_{\bullet}\right)$ let $\mathrm{S}_{\mathrm{A}, \mathrm{M}}: j^{*} \mathrm{~A} \stackrel{\mathrm{L}}{\otimes} \mathrm{D}_{\mathrm{Y}_{\bullet}^{\prime}} j^{*} \mathrm{D}_{\mathrm{Y}}(\mathrm{M}) \rightarrow \mathrm{D}_{\mathrm{Y}_{\bullet}^{\prime}} j^{*} \mathrm{D}_{\mathrm{Y} \bullet}(\mathrm{A} \stackrel{\mathrm{L}}{\otimes} \mathrm{M})$ denote the composite

$$
\begin{aligned}
j^{*} \mathrm{~A} \otimes \mathscr{L} \operatorname{hom}\left(j^{*} \operatorname{Rhom}\left(\mathrm{M}, \omega_{\mathrm{Y}_{\bullet}}\right), \omega_{\mathrm{Y}_{\bullet}^{\prime}}\right) & \simeq j^{*}\left(\mathrm{~A} \stackrel{\mathrm{L}}{\otimes} \mathscr{R h o m}\left(\mathscr{R h o m}\left(\mathrm{M}, \omega_{\mathrm{Y}_{\bullet}}\right), j_{*} \omega_{\mathrm{Y}_{\bullet}^{\prime}}\right)\right. \\
& \rightarrow j^{*} \mathscr{R h o m}\left(\mathscr{R h o m}\left(\mathrm{A}, \mathscr{R h o m}\left(\mathrm{M}, \omega_{\mathrm{Y}_{\bullet}}\right)\right), j_{*} \omega_{\mathrm{Y}_{\bullet}^{\prime}}\right) \\
& \simeq j^{*} \mathscr{R h o m}\left(\mathscr{R} h o m\left(\mathrm{~A} \otimes \mathrm{L} \mathbf{M}, \omega_{\mathrm{Y}_{\bullet}}\right), j_{*} \omega_{\mathrm{Y}_{\bullet}^{\prime}}\right) \\
& \simeq \mathrm{D}_{\mathrm{Y}_{\bullet}^{\prime}} j^{*} \mathrm{D}_{\mathrm{Y}_{\bullet}}(\mathrm{A} \stackrel{\mathbf{L}}{\otimes} \mathrm{M}) .
\end{aligned}
$$

Here the second morphism is given by 5.5.7 and the third morphism is by the adjunction property of $\otimes$.

Lemma 5.6.3. For any $\mathrm{A} \in \mathrm{D}_{c}\left(\mathrm{Y}_{\bullet}\right)$ the map $\alpha_{\mathrm{A}}$ is equal to the composite

$$
j^{*} \mathrm{~A}\langle d\rangle \simeq j^{*} \mathrm{~A} \stackrel{\mathrm{L}}{\otimes} j^{*} \Lambda\langle d\rangle \stackrel{\alpha_{\Lambda}}{\longrightarrow} j^{*} \mathrm{~A} \stackrel{\mathrm{L}}{\otimes} \mathscr{R} h o m\left(j^{*} \operatorname{Rhom}\left(\Lambda, \omega_{\mathrm{Y}_{\bullet}}\right), \omega_{\mathrm{Y}_{\bullet}^{\prime}}\right) \stackrel{\mathrm{S}_{\mathrm{A}, \Lambda}}{\longrightarrow} \mathrm{D}_{\mathrm{Y}_{\bullet}^{\prime}} j^{*} \mathrm{D}_{\mathrm{Y}_{\bullet}}(\mathrm{A}) .
$$

Proof: This follows from the definitions. 
Lemma 5.6.4. For any $\mathrm{A}, \mathrm{B}, \mathrm{M} \in \mathrm{D}_{c}\left(\mathrm{Y}_{\bullet}\right)$, the diagram

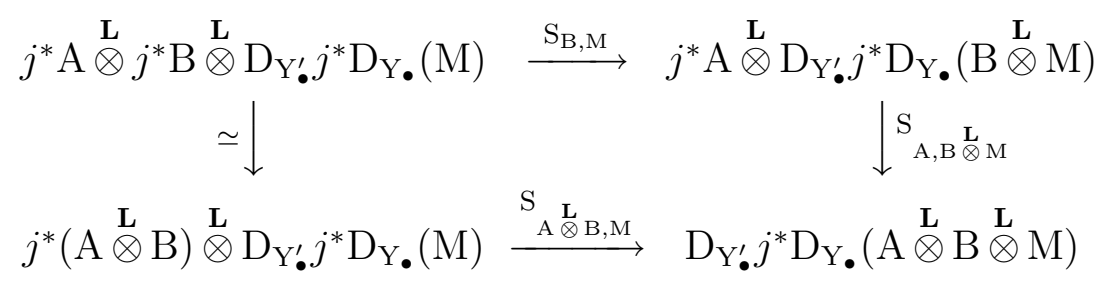

commutes.

Proof: Consider the diagram

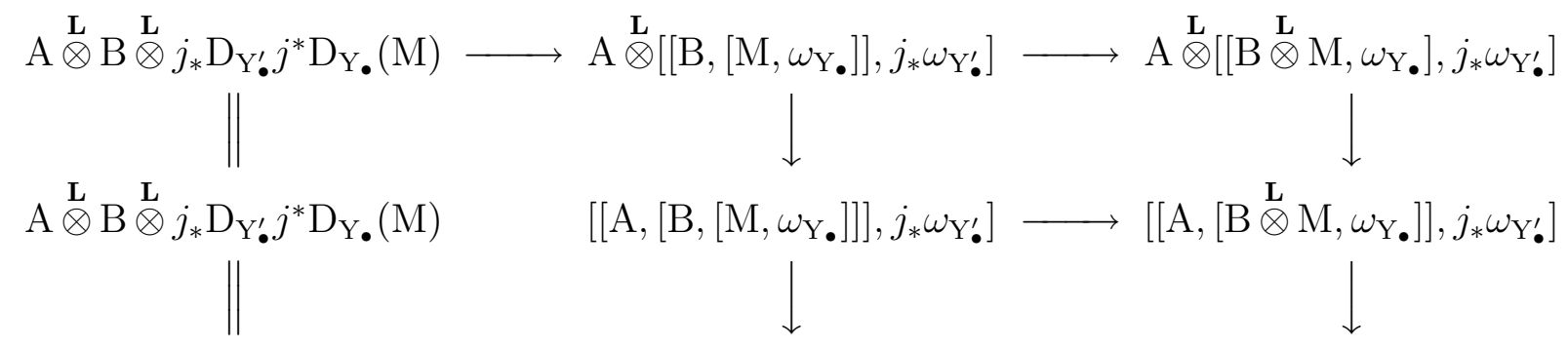

$\mathrm{A} \stackrel{\mathrm{L}}{\otimes} \mathrm{B} \stackrel{\mathrm{L}}{\otimes} j_{*} \mathrm{D}_{\mathrm{Y}_{\bullet}^{\prime}} j^{*} \mathrm{D}_{\mathrm{Y}_{\bullet}}(\mathrm{M}) \longrightarrow\left[\left[\mathrm{A} \stackrel{\mathrm{L}}{\otimes} \mathrm{B},\left[\mathrm{M}, \omega_{\mathrm{Y}_{\bullet}}\right]\right], j_{*} \omega_{\mathrm{Y}_{\bullet}^{\prime}}\right] \longrightarrow\left[\left[\mathrm{A} \stackrel{\mathrm{L}}{\otimes} \stackrel{\mathrm{B}}{\otimes} \mathrm{M}, \omega_{\mathrm{Y}_{\bullet}}\right], j_{*} \omega_{\mathrm{Y}_{\bullet}^{\prime}}\right]$

where to ease the notation we write simply $[-,-]$ for $\mathscr{R} h o m(-,-)$. An elementary verification shows that each of the small inside diagrams commute, and hence the big outside rectangle also commutes. Applying $j^{*}$ we obtain the lemma.

Similarly, for $\mathrm{A}, \mathrm{M} \in \mathrm{D}_{c}\left(\mathscr{X}_{\mathrm{Y}_{\bullet}^{\prime}}^{\prime}\right)$, let $\mathrm{R}_{\mathrm{A}, \mathrm{M}}: i_{*} \mathrm{~A} \stackrel{\mathrm{L}}{\otimes} \mathrm{D}_{\mathscr{X}_{\mathrm{Y}}} i_{*} \mathrm{D}_{\mathscr{X}_{\mathrm{Y}^{\prime}}^{\prime}}(\mathrm{M}) \rightarrow \mathrm{D}_{\mathscr{X}_{\mathrm{Y}}} i_{*} \mathrm{D}_{\mathscr{X}_{\mathrm{Y}^{\prime}}^{\prime}}(\mathrm{A} \stackrel{\mathrm{L}}{\otimes} \mathrm{M})$ be the map

$$
\begin{aligned}
& i_{*} \mathrm{~A} \stackrel{\mathrm{L}}{\otimes} \mathrm{D}_{\mathscr{X}_{\mathrm{Y}_{\bullet}}} i_{*} \mathrm{D}_{\mathscr{X}_{\mathrm{Y}_{\bullet}^{\prime}}^{\prime}}(\mathrm{M}) \simeq i_{*} \mathrm{~A} \stackrel{\mathrm{L}}{\otimes} \mathscr{R} h o m\left(i_{*} \mathscr{R h o m}\left(\mathrm{M}, \omega_{\mathscr{X}_{\mathrm{Y}_{\bullet}^{\prime}}^{\prime}}\right), \omega_{\mathscr{X}_{\mathrm{Y}}}\right) \\
& \simeq i_{*} \mathrm{~A} \stackrel{\mathbf{L}}{\otimes} \mathscr{R h o m}\left(\mathscr{R h o m}\left(i_{*} \mathrm{M}, i_{*} \omega_{\mathscr{X}_{\mathrm{Y}_{\bullet}^{\prime}}^{\prime}}\right), \omega_{\mathscr{X}_{\mathrm{Y}}}\right) \\
& \rightarrow \mathscr{R h o m}\left(\mathscr{R h o m}\left(i_{*} \mathrm{~A}, \mathscr{R h o m}\left(i_{*} \mathrm{M}, i_{*} \omega_{\mathscr{X}_{\mathrm{Y}_{\bullet}^{\prime}}^{\prime}}\right)\right), \omega_{\mathscr{X}_{\mathrm{Y}}}\right) \\
& \simeq \mathscr{R h o m}\left(\mathscr{R h o m}\left(i_{*}(\mathrm{~A} \stackrel{\mathrm{L}}{\otimes} \mathrm{M}), i_{*} \omega_{\mathscr{X}_{\mathrm{Y}_{\bullet}^{\prime}}^{\prime}}\right), \omega_{\mathscr{X}_{\mathrm{Y}}}\right) \\
& \simeq \mathscr{R h o m}\left(i_{*} \mathscr{R h o m}\left(\mathrm{A} \stackrel{\mathrm{L}}{\otimes} \mathrm{M}, \omega_{\mathscr{X}_{\mathrm{Y}_{\bullet}^{\prime}}^{\prime}}\right), \omega_{\mathscr{X}_{\mathrm{Y}_{\bullet}}}\right) \\
& =\mathrm{D}_{\mathscr{X}_{\mathrm{Y}}} i_{*} \mathrm{D}_{\mathscr{X}_{\mathrm{Y}_{\bullet}^{\prime}}^{\prime}}(\mathrm{A} \stackrel{\mathrm{L}}{\otimes} \mathrm{M}),
\end{aligned}
$$


where the third morphism is provided by 5.5.7. As above, one verifies that for A, B, M $\in$ $\mathrm{D}_{c}\left(\mathscr{X}_{\mathrm{Y}_{\bullet}^{\prime}}^{\prime}\right)$ the diagram

$$
\begin{aligned}
& i_{*} \mathrm{~A} \stackrel{\mathrm{L}}{\otimes} i_{*} \mathrm{~B} \stackrel{\mathrm{L}}{\otimes} \mathrm{D}_{\mathscr{X}_{\mathrm{Y}}} i_{*} \mathrm{D}_{\mathscr{X}_{\mathrm{Y}_{\bullet}^{\prime}}^{\prime}}(\mathrm{M}) \stackrel{\mathrm{R}_{\mathrm{B}, \mathrm{M}}}{\longrightarrow} \quad i_{*} \mathrm{~A} \stackrel{\mathrm{L}}{\otimes} \mathrm{D}_{\mathscr{X}_{\mathrm{Y}}} i_{*} \mathrm{D}_{\mathscr{X}_{\mathrm{Y}_{\bullet}^{\prime}}^{\prime}}(\mathrm{B} \stackrel{\mathbf{L}}{\otimes} \mathrm{M}) \\
& \simeq \downarrow \quad \mathrm{R}_{\mathrm{A}, \mathrm{B} \otimes \mathrm{M}} \\
& i_{*}(\mathrm{~A} \stackrel{\mathrm{L}}{\otimes} \mathrm{B}) \stackrel{\mathrm{L}}{\otimes} \mathrm{D}_{\mathscr{X}_{\mathrm{Y}}} i_{*} \mathrm{D}_{\mathscr{X}_{\mathrm{Y}_{\bullet}^{\prime}}^{\prime}}(\mathrm{M}) \stackrel{\mathrm{R}}{\stackrel{\mathrm{A} \otimes \mathrm{L}, \mathrm{M}}{\longrightarrow}} \mathrm{D}_{\mathscr{X}_{\mathrm{Y}}} i_{*} \mathrm{D}_{\mathscr{X}_{\mathrm{Y}_{\bullet}^{\prime}}^{\prime}}(\mathrm{A} \stackrel{\mathrm{L}}{\otimes} \mathrm{B} \stackrel{\mathrm{L}}{\otimes} \mathrm{M})
\end{aligned}
$$

commutes.

From this it follows that if $\varphi_{\mathrm{A}}: \mathrm{A} \rightarrow \mathscr{F}^{\prime}(\mathrm{A})$ denotes the morphism constructed in the proof of 5.5.8, then the diagram

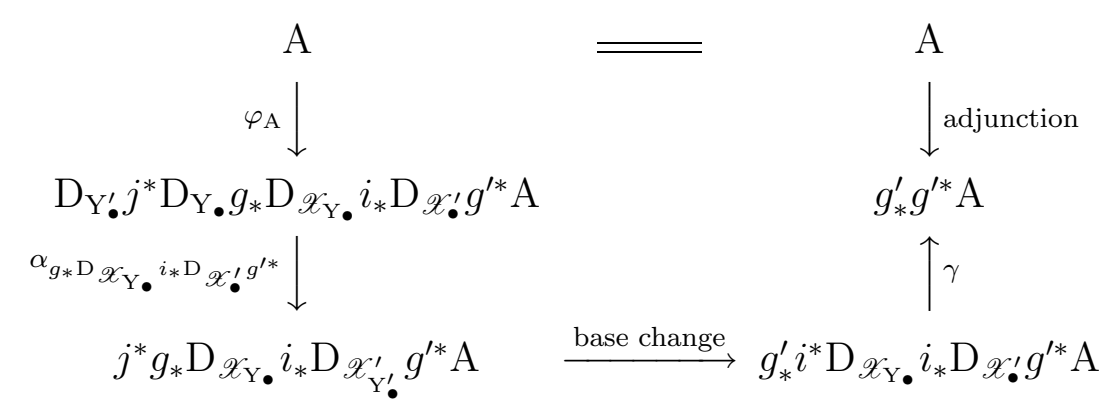

commutes.

Note that the base change morphism in the above diagram is an isomorphism. This can be verified over each $\mathscr{X}_{\mathrm{Y}_{n}^{\prime}}^{\prime}$. Here the functor $\mathrm{D}_{\mathscr{X}_{\mathrm{Y}_{n}}} i_{*} \mathrm{D}_{\mathscr{X}_{\mathrm{Y}_{n}^{\prime}}^{\prime}}$ is up to shift and Tate torsion isomorphic to $i_{!}=i_{*}$. The base change morphism is therefore induced by the isomorphism

$$
j^{*} g_{*} i_{*} \simeq j^{*} j_{*} g_{*}^{\prime} \simeq g_{*}^{\prime} i^{*} i_{*}
$$

By the definition of the morphism in 5.5.8 this implies that for any $\mathrm{A} \in \mathrm{D}_{c}\left(\mathscr{X}_{\mathrm{Y}_{\bullet}^{\prime}}^{\prime}\right)$ the diagram

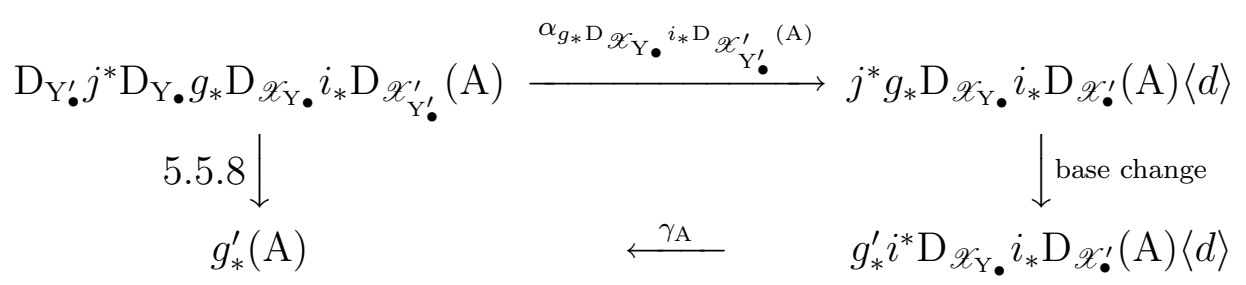

commutes. Combining the commutativity of this diagram with the commutativity of the diagram (verification left to the reader)

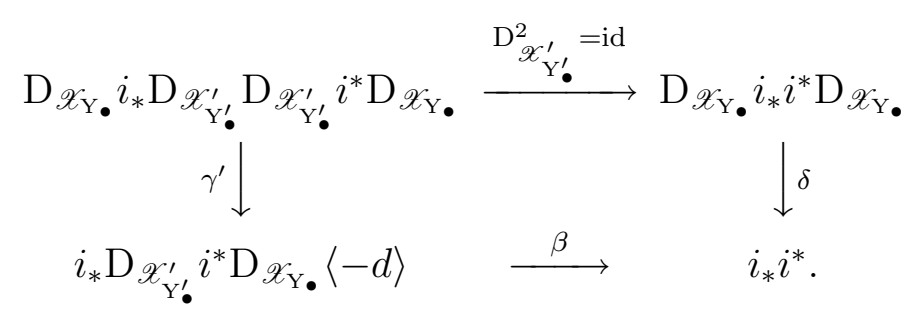


one sees that the diagram

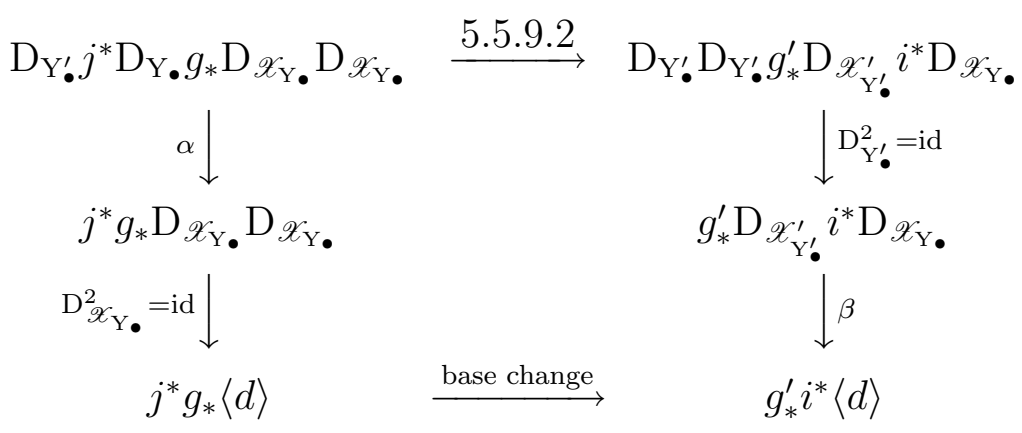

commutes.

We are now ready to prove the equivalences of the two definitions of the base change morphism. The morphism constructed in 5.5 is the composite

$$
\begin{aligned}
\rho^{*} f_{!} & =\rho^{*} q_{*} \mathrm{D}_{\mathrm{Y} \bullet} g_{*} \mathrm{D}_{\mathscr{X}_{\mathrm{Y}} \bullet} \pi^{*} \\
& \simeq p_{*} j^{*} \mathrm{D}_{\mathrm{Y}} g_{*} \mathrm{D}_{\mathscr{X}_{\mathrm{Y}}} \pi^{*} \\
& \rightarrow p_{*} \mathrm{D}_{\mathrm{Y}_{\bullet}^{\prime}} g_{*}^{\prime} \mathrm{D}_{\mathscr{X}_{\mathrm{Y}_{\bullet}^{\prime}}^{\prime}} i^{*} \pi^{*} \\
& \simeq p_{*} \mathrm{D}_{\mathrm{Y}_{\bullet}^{\prime}} g_{*}^{\prime} \mathrm{D}_{\mathscr{X}_{\mathrm{Y}_{\bullet}^{\prime}}^{\prime}} \pi^{\prime *} a^{*} \\
& \simeq f_{!}^{\prime} a^{*}
\end{aligned}
$$

The dual version of this morphism is given by

$$
\begin{aligned}
& \rho^{!} f_{*}=\mathrm{D}_{\mathscr{Y}} \rho^{*} q_{*} \mathrm{D}_{\mathrm{Y}} g_{*} \mathrm{D}_{\mathscr{X}_{\mathrm{Y}}} \pi^{*} \mathrm{D}_{\mathscr{X}} \\
& \simeq p_{*} \mathrm{D}_{\mathrm{Y}_{\bullet}^{\prime}} j^{*} \mathrm{D}_{\mathrm{Y}_{\bullet}} g_{*} \mathrm{D}_{\mathscr{X}_{\mathrm{Y}}} \mathrm{D}_{\mathscr{X}_{\mathrm{Y}}} \pi^{*} \\
& \rightarrow p_{*} \mathrm{D}_{\mathrm{Y}_{\bullet}^{\prime}} \mathrm{D}_{\mathrm{Y}_{\bullet}^{\prime}} g_{*}^{\prime} \mathrm{D}_{\mathscr{X}_{\mathrm{Y}^{\prime}}^{\prime}} i^{*} \mathrm{D}_{\mathscr{X}_{\mathrm{Y}}} \pi^{*} \\
& \simeq \quad p_{*} g_{*}^{\prime} i^{*} \pi^{*}\langle d\rangle \text {. } \\
& \simeq \quad f_{*}^{\prime} a^{!} \text {. }
\end{aligned}
$$

By the commutativity of 5.6.4.1 this is the same as the composite

$$
\begin{array}{rlrl}
\rho^{!} f_{*} & = & \mathrm{D}_{\mathscr{Y}^{\prime}} \rho^{*} q_{*} \mathrm{D}_{\mathrm{Y}_{\bullet}} g_{*} \mathrm{D}_{\mathscr{X}_{\mathrm{Y}}} \pi^{*} \mathrm{D}_{\mathscr{X}} & \\
& \simeq & p_{*} \mathrm{D}_{\mathrm{Y}_{\bullet}^{\prime}} j^{*} \mathrm{D}_{\mathrm{Y}_{\bullet}} g_{*} \mathrm{D}_{\mathscr{X}_{\mathrm{Y}}} \mathrm{D}_{\mathscr{X}_{\mathrm{Y}}} \pi^{*} & \\
& \simeq & p_{*} \mathrm{D}_{\mathrm{Y}_{\bullet}^{\prime}} j^{*} \mathrm{D}_{\mathrm{Y}_{\bullet}} g_{*} \pi^{*} & \mathrm{D}_{\mathscr{X}_{\mathrm{Y}} \bullet}^{2}=\mathrm{id} \\
& p_{*} j^{*} g_{*} \pi^{*}\langle d\rangle & \mathrm{D}_{\mathrm{Y}_{\bullet}^{\prime}} j^{*} \mathrm{D}_{\mathrm{Y}} \simeq j^{*}\langle d\rangle \\
& \rightarrow & p_{*} g_{*}^{\prime} i^{*} \pi^{*}\langle d\rangle & \text { (base change morphism ) } \\
\simeq & f_{*}^{\prime} a^{!} &
\end{array}
$$

From this it follows that the morphism defined in 5.1 agrees with the one defined in 5.5 
5.6.5. The case when $\rho$ is a universal homeomorphism. The same argument used in the previous section shows the agreement of the base change morphism in 5.5 with the base change morphism in 5.4. Indeed the only property of smooth morphisms used in the previous section is that the dualizing sheaves can be described as in 5.6.1.1] This also holds when $\rho$ is a universal homeomorphism (with $d=0$ ).

5.6.6. The case when $\rho$ is an immersion. With notation as in 5.3. note first that to prove that the two base change morphisms agree it suffices to show that they agree on sheaves of the form $\pi_{*} \mathrm{~A}$ with $\mathrm{A} \in \mathrm{D}_{c}\left(\mathscr{X}^{\prime}\right)$. Indeed for any $\mathrm{B} \in \mathrm{D}_{c}(\mathscr{X})$ either base change isomorphism factors as

$$
p^{*} f_{!} \mathrm{B} \stackrel{\mathrm{id} \rightarrow \pi_{*} \pi^{*}}{\longrightarrow} p^{*} f_{!} \pi_{*} \pi^{*} \mathrm{~B} \longrightarrow \phi_{!} \pi^{*} \pi_{*} \pi^{*} \mathrm{~B}=\phi_{!} \pi^{*} \mathrm{~B} .
$$

In order to prove that the two base change morphisms agree, it is useful to first give an alternate description of the morphism defined in 5.3 .

With notation as in 5.3, there is for any $\mathrm{A} \in \mathrm{D}_{c}\left(\mathscr{X}^{\prime}\right)$ a canonical isomorphism

$$
\begin{aligned}
\mathrm{D}_{\mathscr{X}}(\mathrm{A}) & \simeq \mathscr{R h o m}\left(\mathrm{A}, \pi^{!} \Omega_{\mathscr{X}}\right) \\
& \simeq \pi^{*} \pi_{*} \mathscr{R h o m}\left(\mathrm{A}, \pi^{!} \Omega_{\mathscr{X}}\right) \\
& \simeq \pi^{*} \mathscr{R h o m}\left(\pi_{*} \mathrm{~A}, \Omega_{\mathscr{X}}\right) \\
& \simeq \pi^{*} \mathrm{D}_{\mathscr{X}}\left(\pi_{*} \mathrm{~A}\right),
\end{aligned}
$$

and similarly $\mathrm{D}_{\mathscr{Y}} \simeq p^{*} \mathrm{D}_{\mathscr{Y}} p_{*}$. We can also write these isomorphisms as $\pi_{*} \mathrm{D}_{\mathscr{X}^{\prime}} \simeq \mathrm{D}_{\mathscr{X}} \pi_{*}$ and $p_{*} \mathrm{D}_{\mathscr{Y}} \simeq \mathrm{D}_{\mathscr{Y}} p_{*}$.

We therefore obtain a morphism

$$
\begin{aligned}
p^{*} \mathrm{R} f_{!}\left(\pi_{*} \mathrm{~A}\right) & =p^{*} \mathrm{D}_{\mathscr{Y}} f_{*} \mathrm{D}_{\mathscr{X}}\left(\pi_{*} \mathrm{~A}\right) \\
& \simeq p^{*} \mathrm{D}_{\mathscr{Y}} f_{*} \pi_{*} \mathrm{D}_{\mathscr{X}^{\prime}}(\mathrm{A}) \\
& \simeq p^{*} \mathrm{D}_{\mathscr{Y}} p_{*} \phi_{*} \mathrm{D}_{\mathscr{X}^{\prime}}(\mathrm{A}) \\
& \simeq p^{*} p_{*} \mathrm{D}_{\mathscr{Y}} \phi_{*} \mathrm{D}_{\mathscr{X}^{\prime}}(\mathrm{A}) \\
& \simeq \quad \phi_{!} \mathrm{A} .
\end{aligned}
$$

Lemma 5.6.7. This morphism agrees with the one defined in 5.3. In particular the morphism 5.6 .6 .1 is an isomorphism. 
THE SIX OPERATIONS FOR SHEAVES ON ARTIN STACKS I: FINITE COEFFICIENTS

Proof: Chasing through the definitions this amounts to the commutativity of the following diagram

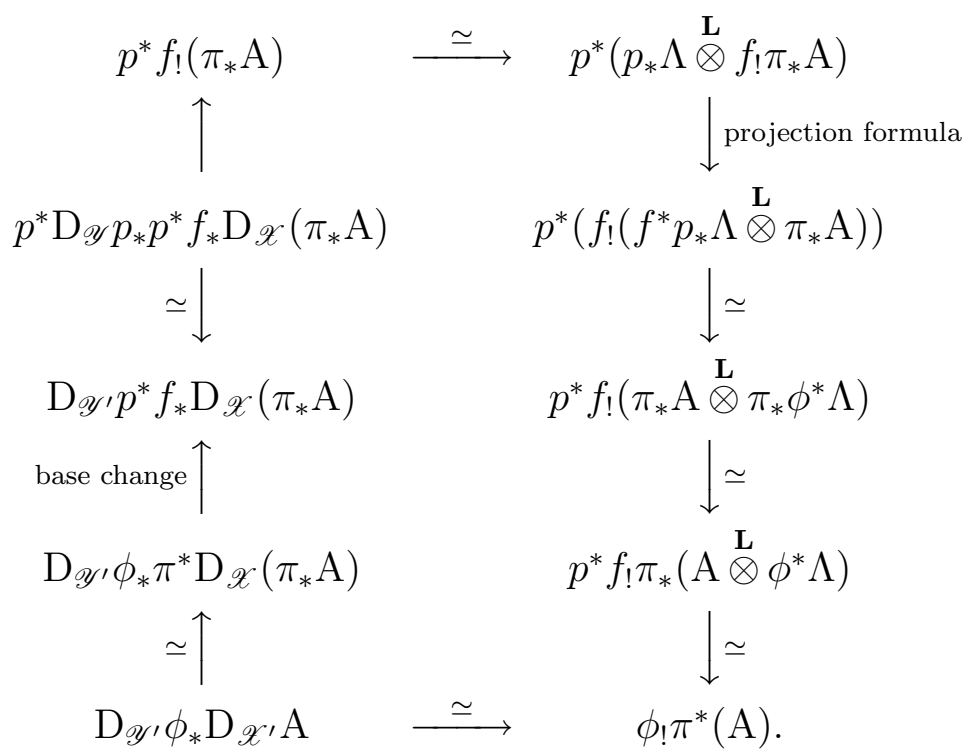

We leave to the reader this verification.

In particular, since the map 5.6.6.1 is an isomorphism we can define the base change morphism for $\mathrm{B} \in \mathrm{D}_{c}(\mathscr{X})$ as the composite

$$
p^{*} f_{!} \mathrm{B} \longrightarrow p^{*} f_{!}\left(\pi_{*} \pi^{*} \mathrm{~B}\right) \stackrel{5.6 .6 .1}{\longrightarrow} \phi_{!} \pi^{*} \mathrm{~B} .
$$

Using this alternate description of the base change morphism in 5.3. we can prove the equivalence with that given in 5.5. By a standard reduction it suffices to consider the case of a closed immersion. So fix the diagram 5.5.6.1 with $\rho$ a closed immersion, and choose a diagram as in 5.5.6.3. Since $\rho$ is a closed immersion we may without loss of generality assume that 5.5 .6 .3 is cartesian.

Lemma 5.6.8. The functors $j_{*}: \mathrm{D}\left(\mathrm{Y}_{\bullet}^{\prime}\right) \rightarrow \mathrm{D}\left(\mathrm{Y}_{\bullet}^{\prime}\right)$ and $i_{*}: \mathrm{D}\left(\mathscr{X}_{\mathrm{Y}_{\bullet}^{\prime}}^{\prime}\right) \rightarrow \mathrm{D}\left(\mathscr{X}_{\mathrm{Y}}\right)$ have right adjoints $j^{!}$and $i^{!}$respectively.

Proof: $\quad$ In fact $j^{!}=j^{*} \mathrm{R} \Gamma_{\mathrm{Y}_{\bullet}^{\prime}}$ and $i^{!}=i^{*} \mathrm{R} \Gamma_{\mathscr{X}_{\mathrm{Y}_{\bullet}^{\prime}}^{\prime}}$.

Note that for any $[n] \in \Delta$, the restriction of $j^{!}$(resp. $i^{!}$) to a functor $\mathrm{D}\left(\mathrm{Y}_{n}\right) \rightarrow \mathrm{D}\left(\mathrm{Y}_{n}^{\prime}\right)$ (resp. $\left.\mathrm{D}\left(\mathscr{X}_{\mathrm{Y}_{n}}\right) \rightarrow \mathrm{D}\left(\mathscr{X}_{\mathrm{Y}_{n}^{\prime}}^{\prime}\right)\right)$ agrees with the usual extraordinary inverse image. This follows for example from the explicit description of these functors in the proof of 5.6.8

Lemma 5.6.9. There are canonical isomorphisms $\omega_{\mathrm{Y}_{\bullet}^{\prime}} \simeq j^{!} \omega_{\mathrm{Y}_{\bullet}}$ and $\omega_{\mathscr{X}_{\mathrm{Y}_{\bullet}^{\prime}}^{\prime}} \simeq i^{!} \omega_{\mathscr{X}_{\mathrm{Y}}}$. 
Proof: By the glueing lemma 2.3.3, it suffices to construct an isomorphism over each $\mathrm{Y}_{n}^{\prime}$ (resp. $\left.\mathscr{X}_{\mathrm{Y}_{n}^{\prime}}^{\prime}\right)$. Let $d$ denote the relative dimension of $\mathrm{Y}_{n}$ over $\mathscr{Y}$. Then $d$ is also equal to the relative dimension of $\mathrm{Y}_{n}^{\prime}$ over $\mathscr{Y}^{\prime}$, the relative dimension of $\mathscr{X}_{\mathrm{Y}_{n}}$ over $\mathscr{X}$, and the relative dimension of $\mathscr{X}_{Y_{n}^{\prime}}^{\prime}$ over $\mathscr{X}^{\prime}$. We therefore have

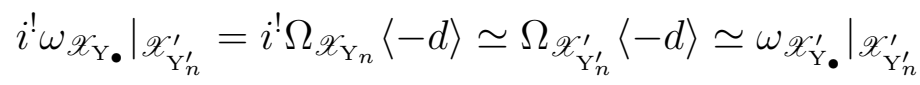

and

$$
\left.j ! \omega_{\mathrm{Y}} \cdot\right|_{\mathrm{Y}_{n} .}=\left.j ! \Omega_{\mathrm{Y}_{n}}\langle-d\rangle \simeq \Omega_{\mathrm{Y}_{n}^{\prime}}\langle-d\rangle \simeq \omega_{\mathrm{Y}^{\prime}}\right|_{\mathrm{Y}_{n}^{\prime}} .
$$

Lemma 5.6.10. For any $\mathrm{A} \in \mathrm{D}\left(\mathrm{Y}_{\bullet}^{\prime}\right)$ and $\mathrm{B} \in \mathrm{D}\left(\mathrm{Y}_{\bullet}\right)$ (resp. $\mathrm{C} \in \mathrm{D}\left(\mathscr{X}_{\mathrm{Y}_{\bullet}^{\prime}}^{\prime}\right)$ and $\mathrm{E} \in \mathrm{D}\left(\mathscr{X}_{\mathrm{Y}_{\bullet}}\right)$ ) we have

$$
j_{*} \mathscr{R h o m}\left(\mathrm{A}, j^{!} \mathrm{B}\right) \simeq \mathscr{R h o m}\left(j_{*} \mathrm{~A}, \mathrm{~B}\right), \quad i_{*} \mathscr{R h o m}\left(\mathrm{C}, i^{!} \mathrm{E}\right) \simeq \mathscr{R h o m}\left(i_{*} \mathrm{C}, \mathrm{E}\right) .
$$

Proof: Since $j^{\text {! }}$ is right adjoint to $j_{*}$, there is an adjunction morphism $j^{!} j_{*} \rightarrow$ id. This map induces a morphism

$$
j_{*} \mathscr{R h o m}\left(\mathrm{A}, j^{\prime} \mathrm{B}\right) \simeq \mathscr{R h o m}\left(j_{*} \mathrm{~A}, j_{*} j_{!} \mathrm{B}\right) \rightarrow \mathscr{R h o m}\left(j_{*} \mathrm{~A}, \mathrm{~B}\right) .
$$

That this map is an isomorphism can be verified after restricting to each $Y_{n}$ in which case it follows from the theory for schemes [4, XVIII, 3.1.10. The same argument gives the second isomorphism in the Lemma.

Corollary 5.6.11. For any $\mathrm{A} \in \mathrm{D}_{c}\left(\mathrm{Y}_{\bullet}^{\prime}\right)$ there is a natural isomorphism $\mathrm{D}_{\mathrm{Y}_{\bullet}} j_{*} \mathrm{~A} \simeq j_{*} \mathrm{D}_{\mathrm{Y}^{\prime}}(\mathrm{A})$, and for $\mathrm{B} \in \mathrm{D}_{c}\left(\mathscr{X}_{\mathrm{Y}_{\bullet}^{\prime}}^{\prime}\right)$ there is a canonical isomorphism $\mathrm{D}_{\mathscr{X}_{\bullet}} i_{*} \mathrm{~B} \simeq i_{*} \mathrm{D}_{\mathscr{Y}_{\mathrm{Y}_{\bullet}^{\prime}}^{\prime}} \mathrm{B}$.

For $\mathrm{A} \in \mathrm{D}_{c}\left(\mathrm{Y}_{\bullet}^{\prime}\right)$, let $\alpha_{\mathrm{A}}$ denote the isomorphism

$$
\begin{aligned}
j_{*} \mathrm{~A} & \simeq \mathrm{D}_{\mathrm{Y}_{\bullet}} j^{*} j_{*} \mathrm{D}_{\mathrm{Y}^{\prime}} \mathrm{A} \\
& \simeq \mathrm{D}_{\mathrm{Y}_{\bullet}} j^{*} \mathrm{D}_{\mathrm{Y}_{\bullet}} j_{*} \mathrm{~A},
\end{aligned}
$$

and for $\mathrm{B} \in \mathrm{D}_{\mathscr{X}_{\mathrm{Y}_{\bullet}^{\prime}}^{\prime}}$ let $\beta_{\mathrm{B}}$ denote the isomorphism

$$
\begin{aligned}
i_{*} \mathrm{~B} & \simeq i_{*} \mathrm{D}_{\mathscr{X}_{\mathrm{Y}^{\prime}}^{\prime}} \mathrm{D}_{\mathscr{X}_{\mathrm{Y}^{\prime}}^{\prime}}(\mathrm{B}) \\
& \simeq i_{*} \mathrm{D}_{\mathscr{X}_{\mathrm{Y}^{\prime}}^{\prime}} i^{*} i_{*} \mathrm{D}_{\mathscr{X}_{\mathrm{Y}^{\prime}}^{\prime}}(\mathrm{B}) \\
& \simeq i_{*} \mathrm{D}_{\mathscr{X}_{\mathrm{Y}_{\bullet}^{\prime}}^{\prime}} i^{*} \mathrm{D}_{\mathscr{X}_{\mathrm{Y}}}\left(i_{*} \mathrm{~B}\right) .
\end{aligned}
$$


Define $\gamma_{\mathrm{B}}^{\prime}$ to be the isomorphism

$$
\begin{aligned}
i_{*} \mathrm{~B} & \simeq \mathrm{D}_{\mathscr{X}_{\mathrm{Y}}} \mathrm{D}_{\mathscr{X}_{\mathrm{Y}}} i_{*} \mathrm{~B} \\
& \simeq \mathrm{D}_{\mathscr{X}_{\mathrm{Y}}} i_{*} \mathrm{D}_{\mathscr{X}_{\mathrm{Y}_{\bullet}^{\prime}}^{\prime}} \mathrm{B}
\end{aligned}
$$

and let $\gamma_{\mathrm{B}}: i^{*} \mathrm{D}_{\mathscr{X}_{\mathrm{Y}_{\bullet}}} i_{*} \mathrm{D}_{\mathscr{X}_{\mathrm{Y}_{\bullet}^{\prime}}^{\prime}}(\mathrm{B}) \rightarrow \mathrm{B}$ be the isomorphism obtained by adjunction.

Following the same outline used in 5.6.1 (replacing the $\alpha$ 's, $\beta$ 's, and $\gamma$ 's by the above defined morphisms), one sees that the morphism 5.5.9.2 in the case of a closed immersion is given by the composite

$$
\begin{aligned}
j^{*} \mathrm{D}_{\mathrm{Y}} g_{*} \mathrm{D}_{\mathscr{X}_{\mathrm{Y}}} i_{*} & \simeq j^{*} \mathrm{D}_{\mathrm{Y}_{\bullet}} g_{*} i_{*} \mathrm{D}_{\mathscr{X}_{\mathrm{Y}^{\prime}}^{\prime}} \\
& \simeq j^{*} \mathrm{D}_{\mathrm{Y}_{\bullet}} j_{*} g_{*}^{\prime} \mathrm{D}_{\mathscr{X}_{\mathrm{Y}^{\prime}}^{\prime}} \\
& \simeq j^{*} j_{*} \mathrm{D}_{\mathrm{Y}_{\bullet}^{\prime}} g_{*}^{\prime} \mathrm{D}_{\mathscr{X}_{\mathrm{Y}_{\bullet}^{\prime}}^{\prime}} \\
& \simeq \mathrm{D}_{\mathrm{Y}_{\bullet}^{\prime}} g_{*}^{\prime} \mathrm{D}_{\mathscr{X}_{\mathrm{Y}_{\bullet}^{\prime}}^{\prime}} .
\end{aligned}
$$

From this it follows that the sequence of morphisms in 5.5.9.3 is identified via cohomological descent with the sequence of morphisms 5.6.7.1. and hence the two base change morphisms are the same.

5.7. Kunneth formula. Let $\mathscr{Y}_{1}$ and $\mathscr{Y}_{2}$ be stacks, and set $\mathscr{Y}:=\mathscr{Y}_{1} \times \mathscr{Y}_{2}$. Let $p_{i}: \mathscr{Y} \rightarrow \mathscr{Y}_{i}$ $(i=1,2)$ be the projection and for two complexes $\mathrm{L}_{i} \in \mathrm{D}_{c}^{-}\left(\mathscr{Y}_{i}\right)$ let $\mathrm{L}_{1} \stackrel{\mathrm{L}}{\otimes}_{\mathrm{S}} \mathrm{L}_{2} \in \mathrm{D}(\mathscr{Y})$ denote $p_{1}^{*} \stackrel{\mathrm{L}}{1}_{1}^{\mathbf{L}} \otimes_{\Lambda} p_{2}^{*} \mathrm{~L}_{2}$.

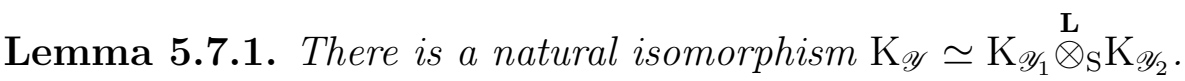

Proof: By ([[5], III.1.7.6) there is for any smooth morphisms $\mathrm{U}_{i} \rightarrow \mathscr{Y}_{i}(i=1,2)$ with $\mathrm{U}_{i}$ a scheme, a canonical isomorphism

$$
\left.\left.\left.\mathrm{K}_{\mathscr{Y}}\right|_{\mathrm{U}_{1} \times_{\mathrm{S}} \mathrm{U}_{2}} \simeq \mathrm{K}_{\mathscr{Y}_{1}}\right|_{\mathrm{U}_{1}} \stackrel{\mathrm{L}}{\otimes_{\mathrm{S}} \mathrm{K}_{\mathscr{Y}_{2}}}\right|_{\mathrm{U}_{2}}
$$

Furthermore, this isomorphism is functorial with respect to morphisms $\mathrm{V}_{i} \rightarrow \mathrm{U}_{i}$. It follows that the sheaf $\mathrm{K}_{\mathscr{Y}_{1}} \mathrm{~L}_{\mathrm{S}} \mathrm{K}_{\mathscr{Y}_{2}}$ also satisfies the $\mathscr{E} x t$-condition (2.3.3), and hence to give an isomorphism as in the Lemma it suffices to give an isomorphism in the derived category of $U_{1} \times_{S} U_{2}$ for all smooth morphisms $\mathrm{U}_{i} \rightarrow \mathscr{Y}_{i}$.

Lemma 5.7.2. Let $(\mathscr{T}, \Lambda)$ be a ringed topos. Then for any $\mathrm{P}_{1}, \mathrm{P}_{2}, \mathrm{M}_{1}, \mathrm{M}_{2} \in \mathrm{D}(\mathscr{T}, \Lambda)$, there is a canonical morphism

$$
\mathscr{R h o m}\left(\mathrm{P}_{1}, \mathrm{M}_{1}\right) \otimes \stackrel{\mathbf{L}}{\otimes} \operatorname{hom}\left(\mathrm{P}_{2}, \mathrm{M}_{2}\right) \rightarrow \mathscr{R h o m}\left(\mathrm{P}_{1} \stackrel{\mathrm{L}}{\otimes} \mathrm{P}_{2}, \mathrm{M}_{1} \stackrel{\mathrm{L}}{\otimes} \mathrm{M}_{2}\right) .
$$


Proof: It suffices to give a morphism

$$
\mathscr{R h o m}\left(\mathrm{P}_{1}, \mathrm{M}_{1}\right) \stackrel{\mathrm{L}}{\otimes} \mathscr{R} \operatorname{hom}\left(\mathrm{P}_{2}, \mathrm{M}_{2}\right) \stackrel{\mathrm{L}}{\otimes} \mathrm{P}_{1} \stackrel{\mathrm{L}}{\otimes} \mathrm{P}_{2} \rightarrow \mathrm{M}_{1} \stackrel{\mathrm{L}}{\otimes} \mathrm{M}_{2} .
$$

This we get by tensoring the two evaluation morphisms

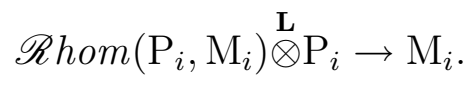

For the definition and standard properties of homotopy colimits we refer to [8].

Lemma 5.7.3. Let $\mathrm{A}, \mathrm{B} \in \mathrm{D}(\mathscr{X})$. Then we have

(1) hocolim $\tau_{\leq n} \mathrm{~A}=\mathrm{A}$;

(2) $\mathrm{A} \stackrel{\mathbf{L}}{\otimes} \mathrm{B}=\operatorname{hocolim} \tau_{\leq n} \mathrm{~A} \stackrel{\mathbf{L}}{\otimes} \tau_{\leq n} \mathrm{~B}$.

Proof: Consider the triangle

$$
\oplus \tau_{\leq n} \mathrm{~A} \stackrel{1-\text { shift }}{\longrightarrow} \oplus \tau_{\leq n} \mathrm{~A} \rightarrow \mathrm{A}
$$

If $\mathrm{C}=$ hocolim $\tau_{\leq n} \mathrm{~A}$ is the cone of $1-$ shift, one gets a morphism $\mathrm{C} \rightarrow \mathrm{A}$. By construction, one has

$$
\mathscr{H}(\mathrm{C})=\lim _{\longrightarrow} \mathscr{H}\left(\tau_{\leq n} \mathrm{~A}\right)=\mathscr{H}(\mathrm{A})
$$

proving that $\mathrm{C} \rightarrow \mathrm{A}$ is an isomorphism. Tensoring $\left(^{*}\right)$ by $\mathrm{B}$ we get therefore a distinguished triangle

$$
\oplus \tau_{\leq n} \mathrm{~A} \stackrel{\mathrm{L}}{\otimes} \mathrm{B} \stackrel{1-\text { shift }}{\longrightarrow} \oplus \tau_{\leq n} \mathrm{~A} \stackrel{\mathrm{L}}{\otimes} \mathrm{B} \rightarrow \mathrm{A} \stackrel{\mathbf{L}}{\otimes} \mathrm{B}
$$

proving

$$
\operatorname{hocolim} \tau_{\leq n} \mathrm{~A} \stackrel{\mathbf{L}}{\otimes} \mathrm{B}=\mathrm{A} \stackrel{\mathrm{L}}{\otimes} \mathrm{B} .
$$

Applying this process again we find

$$
\operatorname{hocolim} \tau_{\leq n} \stackrel{\mathrm{L}}{\otimes} \tau_{\leq m} \mathrm{~B}=\mathrm{A} \stackrel{\mathrm{L}}{\otimes} \mathrm{B}
$$

Because the diagonal is cofinal in $\mathbf{N} \times \mathbf{N}$, the lemma follows.

Proposition 5.7.4. For $\mathrm{L}_{i} \in \mathrm{D}_{c}^{-}\left(\mathscr{Y}_{i}\right)(i=1,2)$, there is a canonical isomorphism

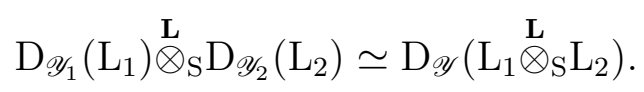


Proof: $\quad$ By 5.7 .1 and 5.7 .2 there is a canonical morphism (note here we also use that $\mathrm{K}_{\mathscr{Y}_{i}}$ has finite injective dimension)

$$
\mathrm{D}_{\mathscr{Y}_{1}}\left(\mathrm{~L}_{1}\right) \stackrel{\mathrm{L}}{\otimes_{\mathrm{S}}} \mathrm{D}_{\mathscr{Y}_{2}}\left(\mathrm{~L}_{2}\right) \rightarrow \mathrm{D}_{\mathscr{Y}}\left(\mathrm{L}_{1} \stackrel{\mathrm{L}}{\otimes_{\mathrm{S}}} \mathrm{L}_{2}\right) .
$$

To verify that this map is an isomorphism, it suffices to show that for every $j \in \mathrm{Z}$ the map

$$
\mathscr{H}^{j}\left(\mathrm{D}_{\mathscr{Y}_{1}}\left(\mathrm{~L}_{1}\right) \stackrel{\mathrm{L}}{\otimes}{ }_{\mathrm{S}} \mathrm{D}_{\mathscr{Y}_{2}}\left(\mathrm{~L}_{2}\right)\right) \simeq \mathscr{H}^{j}\left(\mathrm{D}_{\mathscr{Y}}\left(\mathrm{L}_{1} \stackrel{\mathrm{L}}{\mathrm{S}}_{\mathrm{L}}\right)\right)
$$

Because $\stackrel{\mathbf{L}}{\otimes}$ commutes with homotopy colimits $(\underline{5.7 .3})$, we deduce from $\mathrm{D}(\mathrm{A})=$ hocolim $\mathrm{D}\left(\tau_{\geq} m \mathrm{~A}\right)$ (use 5.7.3) that to prove this we may replace $\mathrm{L}_{i}$ by $\tau_{\geq m} \mathrm{~L}_{i}$ for $m$ sufficiently negative, and therefore it suffices to consider the case when $\mathrm{L}_{i} \in \mathrm{D}_{c}^{b}\left(\mathscr{Y}_{i}\right)$. Furthermore, we may work locally in the smooth topology on $\mathscr{Y}_{1}$ and $\mathscr{Y}_{2}$, and therefore it suffices to consider the case when the stacks $\mathscr{Y}_{i}$ are schemes. In this case the result is [4], XVII, 5.4.3.

Now consider morphisms of S-stacks $f_{i}: \mathscr{X}_{i} \rightarrow \mathscr{Y}_{i}(i=1,2)$, and let $f: \mathscr{X}:=\mathscr{X}_{1} \times_{\mathrm{S}} \mathscr{X}_{2} \rightarrow$ $\mathscr{Y}:=\mathscr{Y}_{1} \times_{\mathrm{S}} \mathscr{Y}_{2}$ be the morphism obtained by taking fiber products. Let $\mathrm{L}_{i} \in \mathrm{D}_{c}^{-}(\mathscr{X})$.

Theorem 5.7.5. There is a canonical isomorphism in $\mathrm{D}_{c}(\mathscr{Y})$

$$
\mathrm{R} f_{!}\left(\mathrm{L}_{1} \stackrel{\mathrm{L}}{\mathrm{S}}_{\mathrm{L}}\right) \rightarrow \mathrm{R} f_{1 !}\left(\mathrm{L}_{1}\right) \stackrel{\mathbf{L}}{\otimes}{ }_{\mathrm{S}} \mathrm{R} f_{2 !}\left(\mathrm{L}_{2}\right) .
$$

Proof: We define the morphism [5.7.5.1 as the composite

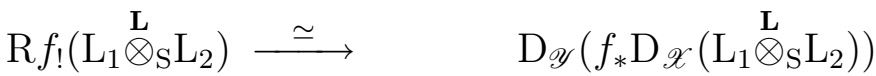

$$
\begin{aligned}
& \stackrel{\simeq}{\longrightarrow} \mathrm{D}_{\mathscr{Y}}\left(f_{*}\left(\mathrm{D}_{\mathscr{X}_{1}}\left(\mathrm{~L}_{1}\right) \stackrel{\mathrm{L}}{\otimes_{\mathrm{S}}} \mathrm{D}_{\mathscr{X}_{2}}\left(\mathrm{~L}_{2}\right)\right)\right) \\
& \longrightarrow \quad \mathrm{D}_{\mathscr{Y}}\left(f_{1 *} \mathrm{D}_{\mathscr{X}_{1}}\left(\mathrm{~L}_{1}\right) \stackrel{\mathrm{L}}{\otimes}_{\mathrm{S}}\left(f_{2 *} \mathrm{D}_{\mathscr{X}_{2}}\left(\mathrm{~L}_{2}\right)\right)\right)
\end{aligned}
$$

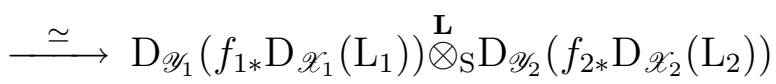

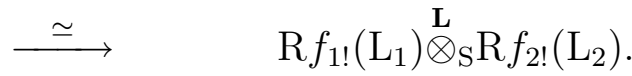

That this map is an isomorphism follows from a standard reduction to the case of schemes using hypercovers of $\mathscr{X}_{i}$, biduality, and the spectral sequences 4.8.1.

\section{REFERENCES}

[1] Revétements étales et groupe fondamental. Société Mathématique de France 2003. Séminaire de Géométrie Algébrique du Bois-Marie 1960-1961 (SGA 1), Dirigé par A. Grothendieck. Augmenté de deux exposés de Mme M. Raynaud, Documents Mathématiques, Vol. 3.

[2] Théorie des topos et cohomologie étale des schémas. Tome 1: Théorie des topos. Springer-Verlag, Berlin, 1972. Séminaire de Géométrie Algébrique du Bois-Marie 1963-1964 (SGA 4), Dirigé par M. Artin, A. Grothendieck, et J. L. Verdier. Avec la collaboration de N. Bourbaki, P. Deligne et B. Saint-Donat, Lecture Notes in Mathematics, Vol. 269. 
[3] Théorie des topos et cohomologie étale des schémas. Tome 2. Springer-Verlag, Berlin, 1972. Séminaire de Géométrie Algébrique du Bois-Marie 1963-1964 (SGA 4), Dirigé par M. Artin, A. Grothendieck et J. L. Verdier. Avec la collaboration de N. Bourbaki, P. Deligne et B. Saint-Donat, Lecture Notes in Mathematics, Vol. 270.

[4] Théorie des topos et cohomologie étale des schémas. Tome 3. Springer-Verlag, Berlin, 1973. Séminaire de Géométrie Algébrique du Bois-Marie 1963-1964 (SGA 4), Dirigé par M. Artin, A. Grothendieck et J. L. Verdier. Avec la collaboration de P. Deligne et B. Saint-Donat, Lecture Notes in Mathematics, Vol. 305.

[5] Cohomologie l-adique et fonctions L. Springer-Verlag, Berlin, 1977. Séminaire de Géometrie Algébrique du Bois-Marie 1965-1966 (SGA 5), Edité par Luc Illusie, Lecture Notes in Mathematics, Vol. 589.

[6] Kai A. Behrend. Derived l-adic categories for algebraic stacks. Mem. Amer. Math. Soc., 163(774), 2003.

[7] A. A. Beulinson, J. Bernstein, and P. Deligne. Faisceaux pervers. In Analysis and topology on singular spaces, I (Luminy, 1981), volume 100 of Astérisque, pages 5-171. Soc. Math. France, Paris, 1982.

[8] Marcel Bökstedt and Amnon Neeman. Homotopy limits in triangulated categories. Compositio Math., 86(2):209-234, 1993.

[9] P. Deligne. Cohomologie étale. Springer-Verlag, Berlin, 1977. Séminaire de Géométrie Algébrique du BoisMarie SGA $4 \frac{1}{2}$, Avec la collaboration de J. F. Boutot, A. Grothendieck, L. Illusie et J. L. Verdier, Lecture Notes in Mathematics, Vol. 569.

[10] Eberhard Freitag and Reinhardt Kiehl. Etale cohomology and the Weil conjecture, volume 13 of Ergebnisse der Mathematik und ihrer Grenzgebiete (3) [Results in Mathematics and Related Areas (3)]. SpringerVerlag, Berlin, 1988. Translated from the German by Betty S. Waterhouse and William C. Waterhouse, With an historical introduction by J. A. Dieudonné.

[11] Edward Frenkel, Dennis Gaitsgory, and Kari Vilonen, On the geometric Langland's conjecture, J. Amer. Math. Soc., 15, 367-417, 2002.

[12] Grivel, P.-P., Catégories dérivées et foncteurs dérivés, In Algebraic D-modules edited by Borel, A., Perspectives in Mathematics, 2, Academic Press Inc., Boston, MA, 1987

[13] Y. Laszlo and M. Olsson, The six operations for sheaves on Artin stacks II: Adic Coefficients, in preparation.

[14] Y. Laszlo and M. Olsson, Perverse sheaves on Artin stacks, in preparation.

[15] Gérard Laumon, Transformation de Fourier homogène. Bull. Soc. Math. France, 131 (4),527-551, 2003.

[16] Gérard Laumon and Laurent Moret-Bailly. Champs algébriques, volume 39 of Ergebnisse der Mathematik und ihrer Grenzgebiete. 3. Folge. A Series of Modern Surveys in Mathematics. Springer-Verlag, Berlin, 2000.

[17] Gérard Laumon and Bao Chau Ngo, Le lemme fondamental pour les groupes unitaires, preprint, 2004.

[18] A. Neeman, The Grothendieck duality theorem via Bousfield's techniques and Brown representability, J. Amer. Math. Soc. 9 (1996), 205-236.

[19] Martin Olsson. Sheaves on artin stacks. preprint, 2005.

[20] C. Serpét Resolution of unbounded complexes in Grothendieck categories. J. Pure Appl. Algebra, 177(1):103-112, 2003.

[21] Jean-Pierre Serre. Cohomologie galoisienne, volume 5 of Lecture Notes in Mathematics. Springer-Verlag, Berlin, fifth edition, 1994. 
[22] N. Spaltenstein. Resolutions of unbounded complexes. Compositio Math., 65(2), 1988.

École Polytechnique CMLS UMR 7640 CNRS F-91128 Palaiseau Cedex France

E-mail address: laszlo@math.polytechnique.fr

University of Texas at Austin Department of Mathematics 1 University Station C1200 Austin, TX 78712-0257, USA

E-mail address: molsson@math.utexas.edu 